(C) Brian Mark Hoffman 1967

All Rights Reserved 
INTERACTIONS BETWEEN MOLECULES AND SUPERCONDUCTORS

\author{
Thesis by \\ Brian Ma Hoffman
}

In Partial Fulfillment of the Requirements

For the Degree of

Doctor of Philosophy

\author{
California Institute of Technology \\ Pasacena, California \\ 1967 \\ (Submitted Angust 4, 1966)
}


To my Father, Mother and Brothers 


\section{ACKNOWLEDGMENTS}

I would like to thank Professon H. M. McConnell for his advice, enthusiasm and for his willingness to listen to a graduate student's pipe-dreams. Dr. D. D. Thomas began this project and is responsible for most of the apparatus. The results reported here are in lange measure the fruition of his efforts. Mr. F. R. Gamble was my co-worker on most of the experiments, and therefore deserves a double measure of credit: one for his competence, the other for his forbearance in putting up with me.

In large measure, it is the contacts I have macie through my graduate studies that have made them enjoyable. I would like to especially mention Dr. Z. G. Soos, Mr. M. H. Itzkowitz, Dr. T. Buckman, Dr. P. L. Nordio, Dr. O. H. Griffitin, Mr. R. M. Metzger and Mr. R. C. Hughes.

My deepest thanks to $\mathrm{Mr}$. R. Young for his painstaking job of proofreading.

Finally, I would like to thank the National Science Foundation for the graduate fellowships which have enabled me to pursue my studies. 


\section{ABSTRACT}

Recently, the possibility has been raised that interactions favorable to superconductivity might occur in moleculcs. Although most molecules or molecular aggregates are not even potentially superconducting, it has occurred to us that molecules can be coupled to metals through various kinds of honds, and that such systems may then have interesting superconducting properties. Our approach to the preparation of such systems involves the deposition of selected molecules on thin metal films.

We first present a discussion of the theory of superconductivity, inciuding a review of some of the interaction mechanisms appropriate to molecules. The experimental apparatus is described. Our results are then reported and discussed. We have found compounds that increase the superconducting transition temperature and critical current of thin vanadium films, as well as others which decrease them, and we have found that the sign of this effect can be correlated in a simple way with the electronic structure of the molecules. The nature of these effects is partially clarified by comparison to similar experiments with indiun filns. 
I. INTRODUCTION . . . . . . . . . . . . I I

II. THEORY OE SUPERCONDUCTIVITY . . . . . . . . 10

Dynamics . . . . . . . . . . . . $=0$

A. Cooper's Probiem . . . . . . . . . . jo

B. Theory of Bardeen, Cooper and Schrieffer . . 15

Interaction Mechanisms ........... 23

A. Plunon-CoupIed Interactions . . . . . . 24

B. Dynamic Charge Transfer . . . . . . . 27

C. Electron-Coupled Interactions ... . . . 29

III. EXPERIVENTS ............... . . 40

Preliminanies ............ . . 40 40

Description of Apparatus . . . . . . . . . 47

A. General Description of Vacuum System . . . 47

B. Pumps . . . . . . . . . . . . . 49

C. Pressure Measurement . . . . . . . . 55

D. Vacuum Evaporation System . . . . . . . . 57

E. Chemicals ............ . 60

F. Substrate Assembly . . . . . . . . . 61

G. Critical Current Measurement . . . . . 65

H. Liquid Helium Temperatures . . . . . . 66

Procedure . . . . . . . . . . . 70

Results ............... 80

A. Film Properties . . . . . . . . . . 80

B. Chemical Effects . . . . . . . . 86

IV. DISCUSSION . . . . . . . . . . . . . 92

REFERENCES ........................ 203

PROPOSITIONS . . . . . . . . . . . . 108 


\section{INTRODUCTION (I)}

Superconductivity was discovered in 1911 by Kämerlingh Onnes (2) during investigations of the changes in resistance of metals as they are cooled. The resistance of most metals he studied decreases rapicily with temperature until some limiting value is reached, and then remains constant with further cooling. However, the resistance of mencury hehaves in this manner only down to $4.15^{\circ} \mathrm{K}$. At this temperature, and within an interval of a fraction of a degree, mercury loses all of its resistance. At that time, this state exhibiting infinite conductivity was only one mystery among the many which puzzied investigators of the properties of metals. The discovery of quantum mechanics and its rapici application to solid state physics (3) permittec a basic understanding of many metailic properties, such as the value of the specific heat magnetic susceptibillty and normal electronic conductivity, but left the nature of superconductivity still à mystery.

For many years after its initiai discovery, the superconducting state was assumed to be basicaliy a state of infinite conductivity, and theoretical efforts were devoted to explaining thic phonomonon. In important step forwarci came in 1933, with the discovery by Meissner and Ochsenfeld (4) that a superconductor is a perfect diamagnet. That is, a magnetic B field is completely excluded from the interior of a massive superconductor, the field penetrating only a distance of $\sim 500 \AA$. Since perfect 
conductivity alone would only prevent a cinange in 3 inside of a superconductor, the Meissner etiect detinitely snows that a superconductor is more than a perfect conductor. It also shows that there is a unique state of a simply connected superconducting body for giver values of the variablco tomporaturc, prcosure and extennally applied B field; the transition from normal to superconducting state is reversible, and themmodynamics may therefore he applied to the system (5).

The general features that a microscopic tineory of superconductivity must embody were first described by F. Iondon in 1935 (6). The unique properties of a superconductor arise because it has an ondered ground state which is to be regarded as a single quanturn state extending throughout the material. A superconductor is "a cuantum structure on a macroscopic scale" which is a "kind of a solicification or condensation of the average monentum distribution." He further statec that his successful phenomenological equations could be interpreted by assuming that "ine electrons are couplec in such a way that the lowest state be separated by a finite interval from the excited states"--in other words, thene is an energy gap in the excitation spectrum (7). Until 1957, however, all attempts to construct a microscopic theory proved to be inadequate. It is perhaps less surprising that forty-six years had to elapse Defore the solution of this problem if its complexity is considered. Whereas the correlation energy due to the coulomb interaction between electrons is of the order of $1 \mathrm{ev} / \mathrm{electron}$, and self-energies associated with the 
electron-phonon interaction are of the orcier of $10^{-4} \mathrm{eV} /$ electron, the energy difference between normal and superconducting states of a metal is only of the order of $10^{-8} \mathrm{eV} /$ electron (8). Since there is no hope of calculating the energy of either state with an accuracy of $10^{-8} \mathrm{eV}$, all that can be done is to attempt to focus on the terms in the energy which cause the difference between superconducting and normäl phases and to directly calculate this difference as accurately as possible.

In 1950, Frohlich (9) suggested that the basic force responsible for superconductivity was an attractive interaction between electrons arising from their interactions with the crystal lattice vibrations (phonons). Although his theory as a whole was inadequate, it predicted an isotope effect in which the transition temperature, $T_{c}$, varied as

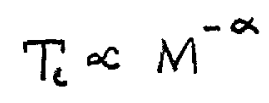

with $\alpha=I / 2$. The discovery by Maxwell (10) and by Reynolds (11) in the same year of an isotope effect in tin, thallium and mercury, with $T_{C} \propto N^{-I / 2 \pm I C \%}$ gave strong support to the importance of an electron-lattice interaction. It is hard to imagine how an isotope effect could fit into a theory based solely on electron-electron interactions, one in which the Born-Oppenheimer approximation is empioyed.

But even with attention focused on the important interaction, a complete theory àid not immediately appear. For one thing, the early theories based on the electron-phonon interaction considered primarily the resulting electron self-energy, 
rather than the true interaction between electrons (12). Another significant reason is that simple perturbation theory cannot be used. As Schafroth proved (13), the Meissner effect cannot be obtained in any finite order of perturbation theory beginning with the uncoupled electron lattice system. Later, Migdal (14) showed that within the perturbation theory there is no energy gap in the electronic excitation spectrum.

The present amazingiy successful microscopic theony of supenconductivity was proposed by J. Bardeen, I. N. Cooper, and J. R. Schrieffer (15) (BCS) in 1957. It is Dased on the fact, established by Coopen (16), that the Fermi sea is unstable in the presence of a net attraction between electrons. It employeci a variational calculation in which the critical term in the hamiltonian was such an attraction; all interactions except this one were assumed to be the same in both nommal and superconducting states. The triaI wave function for the superconducting ground state is described in terms of a linear combination of normal state-like configurations in which the individual electron states are occupied in pairs of opposite spin and momentum. This funktion gives a long range spatial correlation between electrons of opposite spin so as to take advantage of the attractive interaction. The resulting state has an energy gap in the cxcitation spectrum and has the correct thermal and electromagnetic properties to display superconducting behavior. Soon after this, Bogoliubov (17) and Gorkov (18) obtained the BCS results using different formulations. The elegance of their approaches "made 
the field of superconductivity extremely attractive to a great number of theoretical physicists, whose exponentially increasing number of contributions have now flooded the literature" (19). Paralleling the theoretical activity has been a similar burst of experimental work. One indication of this is that in 1962 a compilation of known superconductors contained nine hundred entries, as compared with more than 500 entries in a list prepared in 1960 (20). In the past four years efforts have not slackened. Anong the more exciting occurrences has been the discovery that superconductivity can occur in a semiconductor, GeTe being the first such material found (2I). The search for new materials has largely been made for two different, though relatable reasons. The first has been that by systematically studying the conditions under which superconductivity appears, empirical rules can be set up which correlate the occurrence of superconductivity with the properties of a material. Notable is the work of Matthias (22) who has given general rules for the appearance of superconductivity as related to the number of valence electrons of a metal on alloy. These studies were first undertaken long before the discovery of the BCS theory, in the hope that empirical rules might suggest the basis for a microscopic theory. There are sufficient limitations to the present theory that such experimental studies can still suggest modifications and extensions. Theory has not yet completely reached from the general to the particular, from the idealized superconductor to the particular metal. 
An important instance of this concerns some elements which show a reduced, or even vanishing isotope effect ( $\mathrm{Ru}$, Os, Mo, $\left.\mathrm{Nb}_{5} \mathrm{Sn}\right)(23)$. This has been interpreted to mean that mechanisms other than electron-pinonon interactions can lead to an effective electron-electron attraction. The issue is still unresolved, although more realistic calculations than BCS have been performed. Morel and Ancierson (24) do find a reduced isotope effect; however, their results are now too low, with the exponents $\alpha$ at least 15\% lower than the experimental values for non-transition metals. Garland (25) achieves good agreement with the values of $\alpha$ without invoking a new attractive interaction, and has therefore shown that the vanishing isotope effects do rot preclude the importance of phonons, but he also has elaborated on one of the alternative mechanisms that have been suggested for producing an attractive interaction between electrons in transition metals (25).

In addition, what seem to be anomalously high transition temperatures in certain dilute alloys, particularly iron in titanium (26), and in the elements lanthanum and uranium (27), have also been interpreted as evidence that other mechanisms operate ir addition to phonon coupling. Thus, extending the study of superconductivity into aifferent materials is still a legitimate and necessary test for the existence of alternative mechanisms, since the theory is not suffiriently precise to distinguish quantitatively between the many possible mechanisms which might lead to a similar qualitative result--nameiy, the appearance of superconductivity. 
A second reason for an intensive search tor new superconducting matenials is technological. At present there exists a class of materials which can remain superconducting in high magnetic fieids, ard which have becone very userul in the produchion of supercondicting magnets.

The prospects of Eurthen utilization of superconcuctors in engineering would seem to be unlimited, wene it not fon the lamentable circumstanco that in all tho caoco known to date the superconducting state is reaiized at very low, and therefore difficult to attain, temperatures. Thus the question of the future of supenconductivity is connected primarily with the problem of increasing the critical tem- . peratures, if this is at all possible. (28)

Now, the highest transition temperature presertly known is $18.6 \mathrm{~K}$ for $\mathrm{Nb}_{3} \mathrm{Sn}$. Why the cutoff shoulc come at this temperature is completely unknown. However, it is reasonable trat tnere should be some kind of cutoff if the attractive interaction arises Irom interactions of the conduction electrons with the lattice. One would expect $I_{C}$ to increase as this interaction increases, but only up to a point, for strong electron-lattice interactions can lead to a phase transformation to a new crystal structure with smaller intenactions. Something along these lines is suggested Lil dit examination of the compounds with the highest transition tempenatures, most of which crystallize in the $\beta$-w crystal structure. Several materials which "should" crystailize in this structume and exhibit very high values of $T_{c}$, instead crystallize in a different structure and have lower values of $T_{C}$ than expected. As Niatthias said about one of these, $\mathrm{Zr} \mathrm{r}_{3} \mathrm{Sb}$, "And yet to date it has not been possible to form this compound in the B-W structure by any technique. Perhaps the reason that it cannot 
be made is simply that if this material could be made, its transition temperature would be too higin."

Thus, it is conceivable that the extension of superconductivity to higher temperatures, if that is possible at all, will rest on the occurrence of other mechanisms for establishing an. effective attraction between electrons, and perhaps on the discovery of new kinds of material in which some such mechanism, perhaps already seen in one of the anomalous situations mentioned previously, can be optimized.

The work we have cone was undertaken in the belief that electronic and vibronic interactions favorable to superconductivity could arise in appropriate molecules, and that if the conduction electrons of metal could experience these intenactions by coupling of the molecules to a superconducting metal through various kinds of bonds, such systems should have interesting superconducting properties. We have studied this proposal in a series of experiments in which a chemical is depositec on a thin superconducting film. We have found compounds which increase the transition temperature of superconducting vanadium films, as well as others which decrease it, and we have found that the sign of this effect can be correlated in a simple way with the electronic stnunture of the molecules. Compounds with low electronegativity increase $T_{c}$, while those with high electronegativity decrease it. The nature of these effects is partially clarified by comparison to similar experiments with indium films. 
In section II, a discussion of the microscopic theory of superconductivity is given, with an emphasis on those aspects Which have guided our efforts and are relevant to our expeniments. Section III contains a description of the experimental apparatus, procedure, and results. Section IV is a discussion of these results, and considers in some detail the correlation between molecular properties and effects on superconductivity. 


\section{THEORY OF SUPERCONDUCTIVITY}

\section{Dynamics}

Superconductivity occurs because the normal state of a metal is unstable in the presence of an allraclive inleraction between particles. The ground state of a free electron gas (a usual starting point in the study of metals) corresponds to the complete filling of the one-electron states of wave vector $\underline{\mathrm{k}}$ and

energy $\overline{\mathrm{n}}^{2} \frac{k^{2}}{2}$ up to a certain energy $E=\bar{h}^{2} \frac{k}{2} \mathrm{~m}$, the Fermi energy. However as was first shown by Cooper (16), if there is a net attraction, no matter how weak, between a pair of electrons near the Fermi surface this state becomes unstable and the pain can form a bound state. This important result led directly to the BCS analysis of the superconducting ground state. Although Cooper's result is not in itself a theory of superconcuctivity, for it does not handle the far more complex many-electron problem, it will be reproduced here because it embodies many of the ideas of the full theory in a transparent form. This is then followed by a discussion of lhe BCS tileory.

A. Cooper's Problem.

We consider two interacting electrons above a noninteracting Fermi sea. The effect of the other electrons is to prevent our two electrons from occupying states with $k<k_{F}$ because of the exclusion principle. Since momentum is conserved in the scattering processes due to electron interactions, the wave function of 
the two electrons, $\Psi$, can be expanded as plane wave product functions with identical center of mass momentun, $\underline{k}$. Considering only states where $\underline{\underline{x}}=0, \Psi$ is then only a function of $\underline{\underline{I}}_{1}-\underline{\underline{r}}_{2}$ :

$$
\Psi\left(r_{1}-r_{k}\right)-\sum_{k>k_{j}} g(\underline{k}) \cdot e^{i \underline{k} \cdot\left(r_{1}-r_{1}\right)}
$$

where $g(\underline{k})$ is the probability amplitude for finding one electron in the plane wave state of momentum $\overline{\bar{h}} \underline{k}$ and the other in the state $(-\bar{h} \underline{k})$. The restriction on $k$ in the sumation arises from the exclusion principle. Thus we see that the pain wave function is a superposition of configurations in eaci of which a definite pair state $(\underline{k},-\underline{k})$ is occupied. The calculation is thus an infinite order configuration interaction treatment, with all possibie excited pain states included.

The Schrodinger equation for the two electrons is

$$
-\frac{r^{2}}{2 m}\left(\nabla_{1}^{2}+\nabla_{2}^{2}\right) \Psi_{\left(r_{1}-r_{2}\right)}+1 /\left(r_{1} r_{2}\right) \Psi_{\left(r_{1}-r_{2}\right)}=\left(E+2 E_{p}\right) \psi\left(r_{1}-r_{1}\right)
$$

where $E$ measures the energy of the pair relative to a state with Doth electrons at the Fermi level. Inserting our expression for $\psi$ gives

$$
\left(\frac{h^{2} k^{2}}{m^{2}}-2 E_{p^{\prime}}-\varepsilon\right) g(b)=-\sum_{k^{\prime}>k_{F}} V_{i b^{\prime}} g\left(\underline{b}^{\prime}\right)
$$

where

$$
V_{k k^{\prime}}=\left\langle\underline{k},-\underline{\underline{k}}|V| k_{1}^{\prime}-k^{\prime}\right\rangle=\frac{1}{L^{3}} \int V(r) e^{i\left(\underline{x}^{\prime}-\underline{k}\right) \cdot r} d^{3} r
$$


is the matrix element of the interaction in which the electrons $\left(k^{\prime},-\underline{k}^{\prime}\right)$ scatter off each other into the state $(\underline{k},-\underline{k}) . L^{3}$ is the volume of the system. For $E>0$ this equation has a continuous spectrum of nearly plane-wave solutions. However, if the interaction $V$ is allidive there may also be bound state solutions with $E<0$ and a finite spatial extent. This is demonstrated by considering a simplified interaction which is attractive and constant in an encrgy band thw above the Fermi level

$$
V_{k k^{\prime}}=\left\{\begin{array}{cc}
-\frac{V}{L} & \frac{\hbar^{2} k^{2}}{2 m}, \frac{\hbar^{2} k^{\prime 2}}{2 m}<E_{r}+\hbar \omega=\frac{\hbar^{2} k_{m}^{2}}{2 m}=\frac{i^{2}\left(k+\frac{\delta}{2 m}\right)^{2}}{2 m} \\
0 & \text { otherwise }
\end{array}\right.
$$

Then equation 3 becomes,

$$
\left(\frac{\hbar^{2} k^{2}}{m}-2 E_{p}-E\right) g(\xi)=\frac{V}{L^{3}} \sum_{k>k^{\prime}}^{k_{m}} g\left(k^{\prime}\right)=C
$$

where $C=\frac{V}{L^{3}} \sum g\left(k^{\prime}\right)$ is a constant, independent of $\underline{k}$, giving

$$
g(k)=\frac{C}{\frac{m^{2} k^{2}}{m}-2 E_{p}-E}
$$

Substituting the value for $g(k)$ into the definition of $c$ gives the selt-consistency equation

$$
1=\frac{V}{L^{3}} \sum_{k^{\prime}>k_{p}}^{k_{m}} \frac{1}{\left(\frac{h^{2} k^{2}}{m}-2 E_{p}\right)-E}
$$

Changing the sum to an integral over energy by defining $\epsilon=\frac{x^{2} k^{2}}{2 m}-E_{F}$ and by introducing the density of states per unit energy interval $N(t)$ equation 7 becomes 


$$
I=V \int_{0}^{i n} N\left(t^{\prime}\right) \frac{d t^{\prime}}{2 t^{\prime}-E}
$$

If $\hbar \omega \ll E_{F}, N(\varepsilon)$ can be replaced over the small energy range involved by the constant $N(O)$, the value at the f'ermi level. For a bound state, $-E=\Delta$, a positive quantity, and equation 8 can be simply integrated

$$
\begin{aligned}
1 & =V N(0) \int_{0}^{\hbar \omega} \frac{d t}{2 \epsilon+\Delta} \\
& =V N(0) \ln \frac{2 \hbar \omega+\Delta}{\Delta}
\end{aligned}
$$

For a weak interaction $\mathrm{N}(\mathrm{O}) \mathrm{V}<<1$ and

$$
\Delta=2 \hbar \omega e^{-\frac{2}{N(0) V}}
$$

This is the binding energy of a pair with respect to the Fermi level.

Therefore, in the presence of an attractive interaction we can lower the energy of the system by exciting a pair of electrons above the Fermi level. We would expect from this that in a real system more pairs would go into such a state, causing the normal state to be unstable.

The following observations may be made about the above results:

(I) There is a bound state solution for an attractive $V$ of any magnitude. Furthermore, the binding energy $\Delta$ cannot be 
expanded in powers of $V$ as $V \rightarrow 0$, which shows that this result could not be obtained in any tinite order of perturbation theory. (2) The wave function for the pairs is a true bound state with a large mean square radius and nonexponential dependence for large $\underline{\underline{r}}=\underline{\underline{r}}_{1}-\underline{\underline{r}}_{2}$

$$
\begin{aligned}
& \Psi(t)=\sum_{k+t_{p}}^{k_{m}} g\left(k^{\prime}\right) e^{c k^{\prime} \cdot r} \\
& =\left(\frac{2 \pi}{l}\right)^{3} \int d^{3} k^{\prime} \frac{e^{c k^{\prime} \cdot r}}{6(k)+\Delta} \underset{r \rightarrow \infty}{\infty} \frac{\sin k_{r} r \sin \delta r}{r^{2}} \\
& P=\left[\left\langle r^{2}\right\rangle\right]^{1 / 2}=\left[\frac{\int|\psi(r)|^{2} r^{2} d^{3} r}{\int|\psi(r)|^{2} d^{3} r}\right]^{1 / 2} \sim 10^{-4} \mathrm{~cm} . \\
& \text { for } \frac{\Delta}{k_{a}} \sim 1^{\circ} \mathrm{K}
\end{aligned}
$$

From several considerations, Pippard concluded that the superconducting state is characterized by a long-range coherence such that large changes cannot occur over distances less than $\sim 10^{-4} \mathrm{~cm}$ in pure metals. The extent of these "Cooper Pairs" is of the right order of magnitude to account for this coherence length.

(3) Despite the fact that this is a two-electron calculation, the existence of a localized bound state is nevertheless a direct result of the presence of the other electrons through the exclusion of states with $k<k_{F}$ by the Pauli principle. If the integration could be extended to $h^{2} \frac{k^{2}}{2 m}=0$, for small $V$ there would be no bound state. 
(4) With our simplified interaction, $g(\underline{k})$ depends only on $\varepsilon$. It therefore depends only on $/ \mathrm{k} /$, and the function $\Psi$ is symmetric with respect to the interchange of the two electrons. Thus it must be associated with the antisymetric (singlet) spin function. Although a potential $V_{3 x^{\prime}}$ which is strorgly dependent on the angle between $k$ and $k^{\prime}$ could give rise to bound triplet states, in actuality this appears not to be important.

(5) Recognizing that an instability existo does not necessarily tell what the new state will be. Cooper's treatment is not yet a true explanation of superconductivity for it does not treat all electrons equaly. In fact, it is found that the formation of a "Cooper pair" in the BCS theory depends critically on the presence of other pairs, and the energy gap and most of the observed properties of the superconducting state would be absent were it not for the strong correlations between pairs. Nevertheless, the concept of the correlated occupation of pairs of states with opposite momentum and spin led directly to the BCS theory to be discussed below. The concept of cooper pairs with a lange spatial extension is still an exceedingly useful guide in attempts to undenstand how superconcuctors behave.

B. Theory of Bardeen, Cooper, and Schrieffer

The BCS theory follows the direction pointed by Cooper. The basic mechanism responsible for superconductivity is asoumed to be an effective attractive intenaction between electrons; the crucial assumption is made that all interactions except this 
contribute equally in the superconducting and normal ground states. The many-electron trial Iunction for the superconducting ground state is formed from a linear combination of nearly degenerate normal state-like configurations in which particles are exciled lo Iow energies above the Fermi sed. Their melhod is to choose a subset of configurations whose phases are such that the matrix elements of the interaction between any two configurations $i$ and $j$ are predominantiy negative. Then the trial function for the ground state can be chosen as a Iinear combination of these configurations with coefficients of the same sign. That is, we can write

$$
\psi=\sum_{j} a_{j} \psi_{j}
$$

where each $\psi_{j}$, can be thought of as a particular Slater determinant and the $\hat{a}_{j}$ are to be determined variationally. The energy can then be written, in obvious notation

$$
E=\sum_{j} a_{j}^{2} E_{j}+\sum_{i=j} a_{i} a_{j} H_{i j}
$$

If the $H_{i j}$ vary randomly in sign, the second term gives little. But if they can be made predominantly negative, then the combinaLion of many configurations can give a net lowering of the energy. Now, the effective pain interaction considered by BCS has non-vanishing matrix elements between configurations which ciffer in the occupation of two particlcs, for instance with $k_{1}$ and $k_{2}$ going to $k_{1}{ }^{\prime}$ and $k_{2}{ }^{\prime}$ with conservation of wave vector:

$$
\underline{k}_{1}+\underline{k}_{2}=\underline{k}_{1}^{\prime}+\underline{k}_{2}^{\prime}=\underline{Q}
$$


Because of the Fermi statistics of the electrons, the sign of the matrix element for this scattering depends on the occupation of the other states which are unchanged in the transition. If a definite ordering of all states is chosen, $k_{\alpha}, k_{\beta}, k_{\gamma}, \ldots$. then

$$
\left\langle k_{\alpha}, k_{B}, \cdots k_{1}^{\prime} \cdots k_{2}^{\prime} \cdots k_{\delta}|v| k_{\alpha_{1}} k_{B} \cdots k_{1} \cdots k_{2} \cdots k_{\delta}\right\rangle=(-1)^{N+N^{\prime}}\left\langle k_{1}^{\prime} k_{2}^{\prime}|v| k_{1} k_{8}\right\rangle
$$

(Spin variables are not Indicated)

where $N^{\prime}$ and $N^{\prime}$ are the total number of uccupied slales between $k_{l}$ and $k_{2}$ in the initial state and $k_{1}{ }^{\prime}$ and $k_{2}{ }^{\prime}$ in the final state, respectively. Thus, the relative sign for the matrix element is $(-1)^{N+N^{\prime}}$. Since, for gencral configurations, $N$ and $N^{\prime}$ are equally likely to be even or odd, the sign of these off-diagonal matrix elements will alternate, and they will not add to produce a net lowering of the enengy as in the illustration ahnve. Funthermore, since there are many more matrix elements than configurations, it is not possible to get consistent signs by changing the signs of the coefficients of the various configurations.

The step taken by BCS is to select a subset of configurations (of negligible statistical weight) between which the matrix elements are predominantly negative for an attractive interaction. This is done by associating the states in pairs $\left(k_{1}, k_{2}\right)$, and requiring that in any configuration with one member of a pair occupied, the other is also. Thus $N+N^{\prime}$ must always be even, and the matrix elements all have the same relative sign. To get 
the maximum number of matrix elements, any pair should be able to scatter into any other pair. Since the interaction conserves momentum, all pairs should then have the same total momentum $\mathrm{k}_{1}+\mathrm{k}_{2}=\mathrm{Q}$. By analogy to the Cooper pairs we take the antiparallel spin state. Thus, for the ground state, where $Q=0$, we pair states $(\underline{k} t,-\underline{k} t)$. We have therefore again arrived at the concept of pairs with opposite momentum and spin.

In the trial function chosen by BCS they follow a Hartreelike approximation in which they assume that the probability with which a specific configuration of pairs appears can be expressed as a product of the probability that each indivual pair state is occupied. If $v_{k}$ is the probability amplitude that the state $(k,-k)$ is occupied, and $u_{k}$ the amplitude that it is unoccupied, their values are related by the normalization condition

$$
v_{k}^{2}+u_{k}^{2}=1
$$

The energy difference between the superconducting and normal states is then

$$
W_{i}-w_{n}=2 \sum_{k} \epsilon_{n} v_{k}^{\prime}-2 \sum_{k<k_{p}}\left|\epsilon_{k}\right|+\sum_{k k^{\prime}} V_{k k^{\prime}} u_{k^{\prime}} v_{k} u_{k} v_{k}
$$

where $\varepsilon_{k}$ is the single particle energy for an electron in the state $k$ measured from the Fermi surface and $v_{k}{ }^{\prime}$ is the matrix element

$$
\left\langle-; \underline{k}^{i},-k t ; \cdots|V| k t,-k t ;-\cdots\right\rangle
$$


The interaction tem is of this form because in the initial state pair $k$ must be occupied, amplitude $v_{k}$, and pair $k^{\prime}$ unoccupied, amplitucie $u_{k} ;$ in the final state $k$ must be $u$ ccupied $\left(u_{k}\right)$, and otere: $k^{\prime}$ cccupied $\left(v_{k}:\right)$. The coefficients $u_{k}$ and $v_{k}$, and thus the energy, can be obtainea by variational calculation:

$$
\begin{aligned}
& v_{k}^{\prime}=\frac{1}{2}\left(1+\frac{\epsilon_{k}}{\left[\epsilon_{k}^{2}+\Delta_{k}^{2}\right]^{1 / 2}}\right) \\
& U_{k}^{2}=\frac{1}{2}\left(1-\frac{\epsilon_{k}}{\left[\epsilon_{k}^{2}+\Delta_{k}^{2}\right]^{1 / 2}}\right)
\end{aligned}
$$

where $\Delta_{k}$, the energy gap parameter, is given by the integral equation:

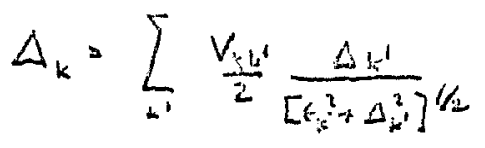

Fon the special case $V_{k^{\prime}}{ }^{\prime}=-V$ for $/ k_{k} /<n \omega$ and zero otherwise, $\Delta_{k}$ is a constant $\Delta$ for $/ \varepsilon /<\hbar \omega$ and zeno for $/ \epsilon_{k} />\pi \omega$. In this case, equation 15 becomes

$$
I=N(0) V \int_{0}^{\pi \omega} \frac{d \epsilon^{\prime}}{\left[\epsilon_{k^{\prime}}^{2}+\Delta^{2}\right]^{1 / z}}
$$

where $N(t)$ ilas been approxindaled as the constanl $N(O)$, upon taking $\hbar \omega \ll E_{F}$. Integrating gives

$$
\begin{aligned}
& \Delta=\frac{\hbar \omega}{\sinh \left(1 / \omega^{\prime}(0) v\right)} \\
& \Delta \approx 2 \hbar \omega e^{-\frac{1}{\pi(01 V}}
\end{aligned}
$$


The simplest excitation from the BCS ground state consists of a state in whlch, for example the state $\underline{k}$ is sureiy cccupled and the state $-\underline{k}$ is surely unoccupied, but otherwise is identica to the ground state. The enercy for this excitation is

$$
E_{x}=\left[\epsilon_{x}^{2}+\Delta^{2}\right]^{1 / 2}
$$

and therefore the single particle excitation spectrum has an energy gap, with minimum excitation eneroy $\wedge$ occuming at the Fermi sunface whene $\varepsilon_{k}=0$.

Extension of the theory to finite tempenatures gives for the transition temperature, $T_{C}$ '

$$
k_{j} T_{c}=1.14 \hbar \omega e^{-\frac{1}{N(a) V}}
$$

This relationship combinec with equation lo gives a general relationship jetween the energy gap at $0^{\circ} K, \Delta(0)$, and $T_{C}$ :

$$
2 \Delta(0)=3.52 k_{0} T_{e}
$$

The range of experimental values for the ratio $2 \Delta(0) / k_{j}$ for all superconductors is 3.2-4.6, with most Iying nean 3.5, affording quite satisfactory agreement.

Again, centain comments are in onder:

(1) This is a theory of superconductivity. In addition to the energy gap and its relation to $T_{C}$, the BCS theory accounts for a second order phase transition at ${ }^{c} c$, for the Meissner effect and probably fon persistent curren-s. A striking prediction of the theory, completely verified by experiment, is the influence 
of coherence effects associated with the pairing on transition probabilities for such phenomena as absorption of ultrasonic or electromagnetic waves and for nuclean spin reiaxation times (30).

(2) Since 1957 an intersive study of the mathematical structure of the theory has occurred. Reformulations (17, 18), more suitable to caiculation have led to a wide increase in the number and kinds of problems considered. However, this brief discussion of tine kincmatics of the thcony, do finst diecoverod by $3 C S$, is sufficient for our purposes, and in fact subsequent theoretical work to be mentioned in later sections was often couched in BCS terms.

(3) As in the Cooper problem, the value of the energy gap, and also of $T_{C}$, cannot be expanded in powers of $V$ for $V \rightarrow 0$.

(4) From equation 13 we see that the contribution of pair state $k$ to the energy of condensation is ( for $k>k_{F}$ )

$$
W_{k}=2 \varepsilon_{k} v_{x}^{2}-V \cdot u_{k} v_{k} \sum_{k^{\prime}} u_{x^{\prime}} v_{k^{\prime}}
$$

Thus, the decrease in energy for a given pair is a result of the interaction with all other pairs, as was stated previously.

(5) If, as was discussed in the introduction, the attmactive interactions arise indirectly from a coupling of the elecLrais lo lite phonons, the cutoff energy, thw, should bo cqual to $k_{B} \theta_{0}$, where $\theta_{0}$ is the Debye temperature, since this is the energy of the most energetic longitudinal phonon. Since $\theta_{0} \propto \mathbb{N}^{1 / 2}$, and $\mathrm{N}(\mathrm{O}) \mathrm{V}$ is expected to be independent of isotopic mass, equation: 18 gives $T_{C} \propto M^{-1 / 2}$, and the general existence of the isotope 
effect is explained. Furthermore, the presence of the exponential accounts for the fact that $T_{C} \ll \theta_{D}$.

(6) However, it is not necessany to assume any panticular mechanism for the attractive interaction. As Iong as a net attraction exists, equation IE has a solution, and a superconducting state is predicted. It is this fact, when buttressed with consicierations of the anomalous cases mentioned in the introduction, which have led peopie to consider altemative attractive interactions . 


\section{Interaction Mechanisms}

Suggestions of non-phonon coupled attractive interactions have been advanced as explanations of centain experimental results, mentioned previously, and also as elements in proposals of unusual synthetic superconducting materials, often predicted to show unusually high transition temperatures (31, 32). Since the experiments described in later sections were undertaker. because of our belief that favorable interactions might result. from the binding of molecules to metals, the nature of such suggestions is of central importance. In this section we first describe the nature of the phonon-rnediated interaction, and then discuss several suggested alternatives, emphasizing how they might occur in metai-molecule systems.

It should bo pointcd out, howcver, that proposing a mechanism is considerably easier than proving its existence in an actual material. Even in the pure metals with small or zero isotcpe effect, the presence of an alternative mechanism is by no means universally accepted. In ailoys, there are many variables which can affect $T_{C}$. As the percentage of alloying component is increased from zero, there is always an initial decrease ir $T_{c}$ caused by elastic impurity scattering (33). At higher concentrations, changes in number of valence electrons, density of states, lattice parameters, and in $\theta_{D}$ all can lead to changes in $\mathrm{T}_{C^{\circ}}$ For example, when titanium is alloyed with vanadium, the transition temperature increases from $\sim \partial^{\circ} \mathrm{X}$ for pure vanadium to $\sim 7^{\circ} \mathrm{K}$ for $80 \%$ 
vanadium, 20\% titanium (34). These changes in $\mathrm{T}_{C}$ correlate quite well with changes in $N(0)$ and $\theta_{D}$ when equation 18 is employed.

\section{A. Yhonon-Loup Led Interactions (y)}

The physical origin of an attractive interaction between electrons, mediated by the lattice, is easily understood. As an electron moves through a lattice in which the ions are not rigidly fixed or infinitely heavy, the lattice will polarize in its wake, producing a temporary excess of positive charge by attempting to crowd nearen to the electron. A second electron traveling with the appropriate phase relative to the first will not see the original lattice potential, but rather the more attractive potential of the distorted lattice, with its excess of positive charge. Thus, the polarizability of the lattice produces an effectively attractive force between two electrons, $v_{p h}$ :

To this force must be added their mutual coulomb repulsion. In systems with high densities of freely moving electrons the long-range coulomb interaction between two electrons is "screened" by the correlated motions of the electrons. A simple model in which screening occurs is a dense free-electron gas with a uniform background charge. Then, by using a linearized form of the Thomas-Fermi equation, a quantum analog to the linearized PoissonBoltzman equation of the Debye-Hitckel theory, the interaction. between two electrons is found to be, not the "bare" interaction. $V_{a} \propto \frac{1}{r}$, but the screened interaction. $V_{c}^{s} \propto \frac{e^{-\lambda r}}{r}$. This is a short-range interaction, with range $\lambda^{-1}=\left(\frac{6 \pi n e^{2}}{E_{p}}\right)^{-1 / 2} \sim .5 A^{0}$. 
Much more complete treatments of screening have been developed by Dolum and Pines (35), and cxtondod by others (35), but the above discussion is qualitatively correct.

Combining these two interactions and taking an average over those states nean the Fermi level which might contribute to the BCS superposition, of configurations, we have an effective matrix element

$$
-V=\left\langle V_{c}+V_{p h}\right\rangle
$$

The oriterion for superconductivity in the RCS thenry is that $-V<0$.

Quantum mechanically, the lattice-mediated interaction is calculated as a second-order process in which a vintual phonon is emitted by one electron and absorbed by another, resulting in an interaction between the two electrons.

The Hamiltonian for the conduction electrons has a term (36)

$$
H_{e-I}=\sum_{i j} v\left(x_{i}-x_{j}\right)
$$

whene $v$ is lle electron-ion interaction, $x_{i}$ is the pooition of the $i^{\prime}$ th electron and $x_{j}$ that of the $j$ th ion. In the BornOppenheimer approximation, $x_{j}=x_{j}^{0}$, the equilibrium position. If motions of the ions are included, $x_{j}=x_{j}^{0}+\& x_{j}$ and the term $\mathrm{H}_{e-I}$ can be expanded to first order in $\delta x_{j}$. This gives a coupling which is first orden in the ion displacements, and which can be written as terms linear in phonon operators. The 
processes caused by these terms are the scattering of an electron from state $k$ to state $k+q$ with either the absorption of a phonon with wave vector $q$, or the emission of a phonon with $-q$. The value of the matrix element for momentum transfer $q, D_{q}$, can be taken for convenience as a constant, D.

The influence of the phonons on the electrons can be studied by a canonical transformation on the Hamiltonian

$$
\tilde{H}=e^{i S} H e^{-i S}
$$

where $S$ is an hermitian operator chosen so that the transformation eliminates terms linear in the phonon variables--terms linear in D. This was first done by Frobizich $(9,36)$, who found that the transformation produced a term corresponding to an interaction between electrons. For a process which can be indicated schematically

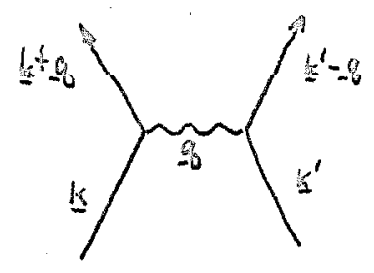

the matrix element is

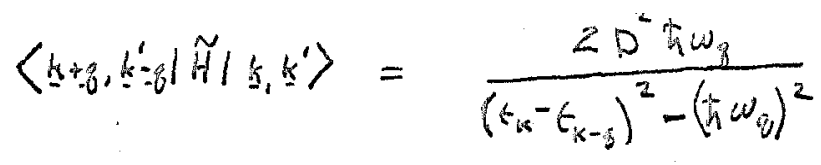

Tt can he seen that for energy diffenences $/ \varepsilon_{k}-\varepsilon_{k-q} /<h w q$, the interaction is attractive (negative). This attractive 
interaction is the result of an exchange of a virtual phonon of wave vector 4 . It is a virtual process because the condition for an attraction shows that the change in electronic energies is not sufficient to create a real phonon of energy hw ${ }_{\mathrm{C}}$. This means that the energy of the intermediate state containing the vintual phonon must be uncertain to approximately $\Delta \mathrm{E} \sim \mathrm{hw}_{\mathrm{q}}$, and that therefore, by the uncertainty principle, its lifetime must be $\tau \leq \frac{1}{s q}$, the period of osciltation of the phonon.

Since most Iongitudinal phonons have energies $h w_{q} \sim k \Theta_{D}$, the approximation of treating the interaction as a constant $-V$ in the energy shell for which the interaction is attractive leads to the condition $/ \varepsilon_{k}-\varepsilon_{k-q} /<k \Theta_{D}$. Then, since the $\varepsilon_{k}$ of importance are of the order $\varepsilon_{k} \sim k_{S} T_{C}$, whereas $\theta_{D}$ is usually greater than $100^{\circ} \mathrm{K}$, it is a good approximation to simplify the energy condition to $/ \epsilon /, \mid \epsilon_{k-q} /<k \theta_{D}$, giving a cutoff $h w=k \theta_{D}$ in equation 18, and to approximate the attractive interaction by the constant

$$
V_{p h}=\frac{2 D^{2}}{k \theta_{D}} \quad\left|\epsilon_{n}\right|,\left|\epsilon_{x-y}\right|<k \theta_{\nu}
$$

To this must be added some average value for the coulomb repulsion.

B. Dynamic Charge Transfer

One of the first departures from the simplified model of a metaI used by BCS was made by SuhI, Matthias, and Walken (37). In an attempt to discuss transition metals, they discussed a twoband model of a metal, with phonon-coupled interactions. Kondo (38) later considered a similar two-band model, but he suggested 
a new mechanism. In the presence of inter band scattering due to s-d exchange interactions, a process independent of phonons, he showed that $\mathrm{T}_{\mathrm{c}}$ is always raised over that of a single attractive band, even when the net interaction between electrons in the second band is repulstve. If both phionon and exchanye mechanisms contribute, he finds that $v$ in the exponent for the transition temperature (equation 18 ) is augmented by a term $V^{\prime}$ due to exchange, incrcasing $T_{C^{*}}$. In the limit that the phonon interaction disappears, he still finds a superconductive solution caused solely by the interband interaction, with

$$
k_{b} T_{c}=1.14 \Delta e^{-\frac{1}{N_{s} \sigma^{2} / 4}}
$$

where $\Delta$ is a quantity related to the width and position of the superconducting $s$-band and $\mathrm{N}_{\mathrm{s}}$ is its density of states, $\mathrm{J}$ is the exchange interaction, and $U$ is the repulsive interaction in the second band (d).

Kondo interprets this result in terms of a second-order virtual process in which a pair in the first band is transferred to the second band and then returis. "Thle secund order process always acts as an attraction in the s-band, regardless of the sign of $\mathrm{J}$. The effective interaction is quite naturally expressed by $\mathrm{J}^{2} / \mathrm{u}$." Thus he arrives at a mechanism for supcrconductivity depending only on the coulomb interaction between electrons. He further considers the case where a narrow d-band is empty, but very close to the Fermi level. If the band is infinitely sharp and located a distance $\Gamma$ above the Fermi level, 
then the transition temperature is given by

$$
k_{B} T_{c}=1.14 \hbar \omega \operatorname{erp}\left(-\frac{1}{N_{0}\left[v+\frac{J^{2}}{u^{\prime}+\frac{r}{3}}\right]}\right)
$$

where $U^{\prime}$ ' is a modified $d$ band repulsion and $Z$ is the total number of electrons per atom which can be accommodated in the d-band. Thus, the interaction with an empty, but accessible band can enhance superconductivity.

Kondo advances his mechanism as an explanation for the reduced isotope effect, and for the unusually high transition temperature of lanthanum (empty f-band near the Fermi level). The same process might operate in a system containing appropriate molecules bonded to a metal. The most promising class of molecules is the planar aromatics because of their ability to form $\pi$-bonds to metal atoms as in the metallocenes (sy). Lf there were unoccupied orbitals of the molecules at energies near the Fermi level, "dynamic charge transfen" processes could occur, in which pairs of conduchion elechrons are vinlually lidsisferred lo the molecule and then return, giving rise to an enhancement of superconductivity.

\section{Electron-Coupled Interactions}

I cnitical extension of the two-band model was made by Garland (25). He assumes a model with one band of nearly free, s-electrons, and a second, tightly bound d-band. His calculation shows that under certain conditions this model admits superconductivity without the inclusion of electron-phonon interactions or 
of interband scattering terms. His theory, which is based on the Hamiltonian for coulomb interactions screened by a frequency dependent dielectric constant $\epsilon(c, w)$, generates an attractive interaction between conduction electrons. The "heavy" d-eiectrons are apparently polarized by the s-electrons and tend to antishield-change the sign of--the interactions of the s-electrons with one another. Thus, he arrives at the possibility of the occurrence of an attractive interaction between electrons caused by the mediation of a distinct second group of electrons, rather than by phonons.

An interaction. of this type, viewed in terms of the virtual excitation of a localized electronic state by ore eiectron and its deexcitation by a second, has been a basic motivating idea in our experiments; we consider it possible that, if a finite concentration of molecules can be coupled to a metal, molecular electronic states can form the second system which antishields the conduction electrons.

In a metal-molecule system, it is also possibie to consider intermediate virtual states other than those associated with an isolated molecule. If the molecule is strongly bound to a metal. atom, or atoms, there may be low-lying excited states of this combined system, including charge-transfer states, which can be virtually excited by the conduction eiectrons. Such considerations are again applicable to aromatic molecules, but may je most important in those molecules which can function as chelating agents for metals atoms in low-valence states (40) through 
"synergic" bonds (4I), such as phenanthrolines, bipyridines, and aromatic arsines.

In addition, McConnell (3I) has proposed a variation of the electron-coupling mecharism which should be particuiarly effective in certain metal-molecule systems. He envisages a situation in which some of the molecular orbitals are "corductively conjugated" to the metal. That is, they are quantum mechanicaily mixed with the conduction band of the metal, aliowing conduction ciectrono to flow through the molecule. At the same time, other orbitals remain largely localized; these supply the system of states for the virtual excitation.

An idealized example is pysazine bonded in a vanadiun lattice:

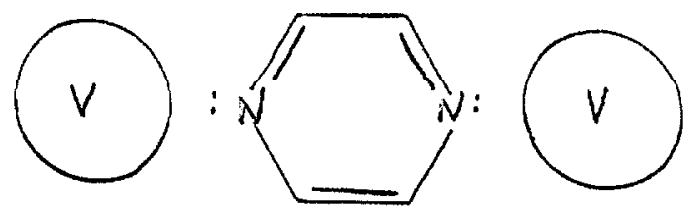

Of the six $\pi$-orbitals of pyrazine, the highest and the lowest are considered to be too far from the Fermi level to be important. Of the remaining four, two are symmetric with respect to reflection in the plane perpencicuiar to the ring asd contialning lhe nitrogen atoms; these have large amplitudes or the nitrogen atoms. Since it is well known that pyridine-type nitrogen atoms form strong bonds to transition metals, these orbitals should be conductively conjugated with the metal. The two antisymmetric orbitals, nowever, have a node at the nitrogen atoms, and should 
remain largely localized. These latten two states form the system necessary for the electron-coupled attractive mechanism.

\section{ANTI SYM}
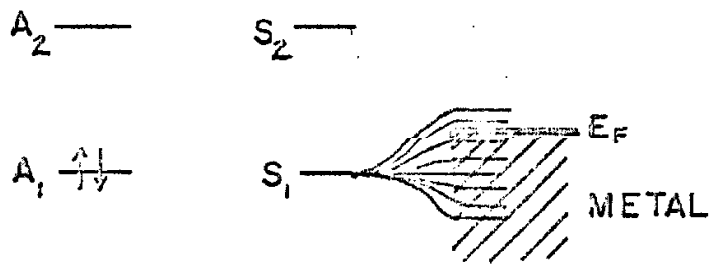

The coupling between conduction electrons and localized states should be very strong because both have finite amplitudes on the carbon atoms of the molecule, giving rise to one-center terms in any matrix element between them. A rough numerical calculation by McConnell indicates that a $T_{c}$ of the order of room temperature might occur when the volume fraction of such a molecuie is roughly $9-10 \%$.

It is obvious that a real situation will probably not be so simple--localized antisymmetric orbitals with infinitely sharp energies. Perlaps mure inpurian, lie medianism is crilically dependent on the relation of the molecular energy levels to $E_{F}$. The states important to superconcuctivity lie within severa $\mathrm{kT}$ of the Fermi level. If the band of conduction states to which $S_{I}$ conjugates does not approach this closely to $E_{F}$, this mechanism cannot operate. Then, redrawing part of the above energy diagram,

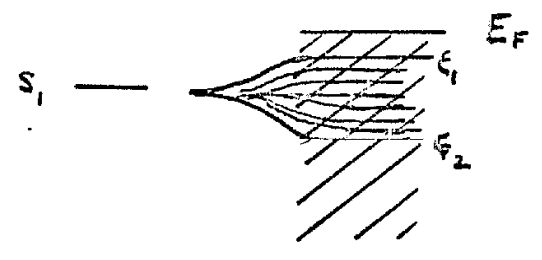


conductive corjugation, to be effective, requires approximately that $E_{F}^{-\varepsilon_{I}} \lessgtr 4 \mathrm{KT}_{C}$, and will work best if $S_{I}$ is nearly degenerate with the Fermi level.

It should be pointec out that under this condition, the density of states, $N(0)$, Is increased by the addition of states near the Fermi level. This will increase $T_{c}$ through equation 18 independently of any virtual electron-coupling. On the other hand, if $I_{F}-\varepsilon_{I} \gg 4 T c$ and if $S_{Z}$ is far above the rermi level, then considerations by M. H. Cohen, in a paper to be discussed again below (42), suggest that a large increase in the density of statcs can arise below the d-band of a transition metal, and that this can be accompaniec by a decrease of the density of states at the Fermi level. Under these conditions, mixing of molecular orbitals with conduntion hard states might cause a decnease of $\mathrm{T}_{\mathrm{C}}$

The mechanism of virtual electronic coupiing has been discussed in detail as part 'of a proposal, made by W. A. Little (32), which has excited the imagination of much of the scientific community. His idea is relatively simple. He considers a Iinear conjugated polyene with altennating single and couble bonds. Attached to this spine are regularly spaced, highly polarizable side-chains with a low-iying excited level of excitation energy hw. The spine electrons are considered to be nearly free. The side-chain electrons are tightly bound, since they are not conjugated to the spine, nor to one another. Little derives an interaction between spine and side-chain in winch a spine electron is 
scattered, either creating or absorbing an electronic excitation (exciton) in the side-chain system. The operators for side-chain excitations appear in the same manner as do phonon operators in the first order interaction between electrons and the lattice. Terms linear in the exciton operators can then be eliminated by the same type of Frohlich canonical transformation, leading to an interaction between electrons of the spine. If the matrix element for this interaction is taken to be an average constant, as in the phonon case, we arrive at an attractive interaction with matrix element

$$
-V_{e}=-\frac{2\left\langle V(a)^{2}\right\rangle}{h \omega_{e}} \quad ;\left|\epsilon_{x}\right|,\left|\epsilon_{k \pm g}\right|<\hbar \omega
$$

where $V(Q)$ is the interaction between spine and side-chain corresponding to $\mathrm{D}_{\mathrm{q}}$ for phonons. This attractive interaction is caused by the fact that one electron moving near the side chain polarizes it in such a way that a considerable positive charge is induced near the spine. This induced charge is experienced by a second electron as an attraction for the first. Quantum mechanically, the interaction is caused by a virtual exchange of excitons. If this term dominates the screened coulomb interaction, then within the framewnrk of the BCS theory, the model is predicted to exhibit superconductivity, with $T_{C}$ given by

$$
k T_{c}=1.14 \hbar \omega_{e} e^{-\frac{1}{M_{(d)} V_{e}}}
$$


The excitement over Little's proposal is largely due to the result of a numerical calculation on a particular model. He finds that the attractive interaction does dominate, and that the polymer might have a transition temperature as high as 2,200 $\mathrm{k}$ ! This large transition temperature results as does Mcconnell's because the pre-exponential factor, $\hbar w$, in equation 18 is no longer $\sim 10^{2 \circ} \mathrm{K}$, but is a molecular excitation energy $\frac{\bar{r} w e}{k_{B}} \sim 10^{4 \circ} \mathrm{K}$. On, more glibly, we have replaced a lattice oscillator by an electronic osciliator. The isotope effect for a phonon-coupled superconductor gives $T_{C} \sim M^{-I / 2}$, where $M$ is the isotopic nuclear mass. Then, for an electron-coupled superconductor, $T_{C}$ should be higher by about the ratio $\sqrt{M / m} \sim 300$, where $m$ is the electronic mass. One reaction to Little's proposal has been the appearance of several papers utilizing variants of it (43). Another reaction has been the appearance of papers criticizing his results. A fundamental objection has been raisec as to the possibility of superconductivity in one dimension (44). This we will not discuss, for our experiments are concerned with the possible occurrence of the eiectronic coupling mechanism in a three dimensional system. Objections have been raised, howeven, which do concern us, for they question the very possibility of utilizing an electron-coupled interaction anc are therefore often equally applicable to our system as to Little's. Keldysh (28) points out that Little's estimates of $\mathrm{V}$ are based on perturbation theory, but that the energy of interaction 
between spine electron and polarized sicie-chain is much greater

Lian lie excilabion energy of the side-cliain excited level.

It is cuite clear that this circumstance should lead to a considerable shift of thcoe lcvelo, and to a cardinal realignment of the side chain, and perhaps of the entire molecule as a whole. Therefore, inttle's calculations show that the molecular model proposec by him is apparently unstable and should rearrange itself spontaneously into some other state. It is not at all obvious here that this new state will be superconducting, and rot dielectric. (28)

Merrifield (45) believes that, for a one-dimensional system, the new state may be semiconducting and ferroelectric, rather than. superconducting.

A second objection, raised by Paulus (46), is concerned with the use of a screened coulomb repulsior. between conduction electrons when one is considering an electron-coupled interaction. Since the virtual electronic oscillator excited by the first electron has a much shorter lifetime than would a lattice oscilIator, the second electron must "follow" the first more closely. If it must be closer than approximately a screening lengtin, use of the full coulomb interaction is claimed to be necessary. Tris question is of crucial impontance, for the assumptions about screening completely determine the sign of the tota- interaction; a screened repulsion can be dominated by the attractive intenaction, an unscreened repulsion cannot. Unfortunately, Paulus makes littie effort to justify his assumption that the inciusion of screening is not appropriate.

The nature of the problem can perhaps be clarified as follows. A virtual excitation of a level with energy hw must 
occur for a time $\tau \leqslant \frac{1}{\omega}$. For a lattice oscillator, $\mathrm{RW_{L }} \sim 10^{-2} \mathrm{eV}$ and $\tau_{L} \lesssim 10^{-13}$ seconds; ton an electronic oscildator, $h w_{e} \sim 2 \mathrm{eV}$ and $\tau_{e} \lesssim 10^{-15}$ seconds. Now an eiectron at the Fermi level moves with a velocity $V_{F}$, where $E_{F}=\frac{V_{F}^{2}}{2 m^{*}}$. If $E_{F}=5 \mathrm{eV}$, and the free electron mass is used, then $v_{F} \sim 10^{7} \mathrm{~cm} / \mathrm{sec}$. Therefore, an electron can move roughly a distance $\dot{a}_{F}=v_{F} \tau$ in the lifetime of a virtual excitation. For a lattice oscillator, $d_{L} \simeq 10^{-6}$. $\mathrm{cm}$. which is mucin groatcr than the ocrocning iongth. However, for an electronic oscillator, $\dot{c}_{e} \sim 10^{-8} \mathrm{~cm}$, which is greater than, but of the same order as a screening length. Thus, if these considerations are appropriate, it would seem that the question of screening in this case lies in the difficult intermediate region in which only a careful analysis can decide the proper interaction, and in which individual cases must be considered. Aluminum, indium and tin all have $\mathrm{v}_{F} \sim 10^{8} \mathrm{~cm} / \mathrm{sec}$; $\mathrm{Nb}_{3} \mathrm{Sn}, \mathrm{Nb}_{3} \mathrm{Ga}$, with lärge effective masses, have $\mathrm{v}_{F} \sim 10^{6} \mathrm{~cm} / \mathrm{sec}$. It should be remembered, however, that Garland's work (25) using a self-consistent treatment of the frequency dependent dielectric constant, indicated that in transition metals an attractive interaction in one group of electrons could be caused by a second group.

Perhapo an even stronger indication of the oubtlctico involved in attempting to invent new superconcuctive mechanisms is seen in a paper by Morrel Cohen (42), considering the four combinations of transition metals anc simple metals as solute and solvent in dilute aIloy systems. For the case of a transition 
metal dissolved in a simple metal, he considens a solute atom with two levels, excitation energy $h \nu$, and ailows the conauction electrons to interact with the soiute. Since the only solute property of importance is the fact that it has two levels, the situation is identica? to that proposed by us for molecules in metals, and is very similar to that in ifttle's polymen. A - straightforward treatment of the conduction electron-impurity interaction would lead to an attractive intenaction between the concuction electrons (47).

However, conen, treating the impurity interaction as a perturbation on the metal states cbtains two new interaction terms. One term is an antishielding of the coulomb interaction, as expected. Taking an average constant, as before,

$$
\Delta V_{c} \sim-\frac{C_{0} C}{4 V} V_{c}<0
$$

where $C_{D}$ is the impunity concentration, and $C$ an "uninteresting" (sic) constant. But the second term is an antishielding of the attractive phonon interaction, giving rise to a repulsion:

$$
\Delta V_{p h} \sim-2 \frac{c_{0} C^{\prime}}{h \nu} V_{p h}>0
$$

Since $/ v_{p h} />/ v_{c} /$ for an ordinary superconductor, and since Cohen claims that $C \sim C^{\prime}$, the new repulsive term is stronger than the new attractive term. The result is a decrease in the net attractive interaction and a lowering of $\mathrm{T}_{\mathrm{C}}$.

This second, and dominating term is absert in all other treatments of similar models. It will not arise if both electron- 
phonon and electron-impurity (or electron-exciton) interactions are treated equaliy as first order interactions and are straightforwardly eliminated by a canonical transformation. It apparently arises when the phonon interaction is treated finst, and then the impurity is used to modify the resulting interaction. Once again, this seems to be a situation where the particular system must be - considerea; the order in which interactions are to be treated can only be justified by very careful, anc probabiy very compiex, analyses.

In sumary, several plausible mechanisms have been described for producing an attractive interaction between electrons, but their theoretical justifications have been questioned. The situation is resolvable either by more detailed calculations, on by successful experiments. It is hoped that the experiments that we will describe offer some pertinent data for such a resolution. 


\section{EXPERIMENTS}

\section{Preliminaries}

Our experimental goal, motivated by a consideration of the various mechanisms which have been discussed, is the preparation of a material in which a metal is coupled to a finite concentration of molecules of an appropriate type. If this can be done in such a. way that the metalific state is nol ciesloyed Ly lie mulecules, while the molecules retain their incividual identity, the material is expected to exnibit interesting properties, both in the supcrconducting, and presumably also in the nommal state.

An ideai method for preparing such a material would be vapon codeposition of a metal and of a small concentration of alloying molecules. This is quite analogous to the matnix isolation technicues of preparing rolecules in rare gas solids (48); in the present case, the matrix would be tine chosen metal. This procedure, however, involves too many parameters whose importance cannot be weighed in advance. The exact molecular properties which are desinable, anc those molecules which best enbody them, cannot be unambiguously predicted. The concentration range of interest must be exceedingly narrow; Eor instance, there must certalniy de some concentration of molecules, beyon whidh line metallic characten of the matnix will be lost. The possibility of molecular decomposition during deposition must be considered. In addition, thore aro all the othen factors mentioned at the 
beginning of Section II which can affect $T_{c}$, possibly masking the presence of a new mechanism.

For the first explonatory experiments designed to measure the effects of organic molecules on the superconductivity of metals, we chose a less ambitious, but workable technique. From adsorption and catalysis studies it is known that strong electronic interactiono, otrong bonds, car occur botwcen molecules and clean metal surfaces ( 49 ). We therefore decided to study thin films of metaliic supercorductors and to look for changes. in supenconcuctive properties protuced hy the deposition of selected chemicals on clean film surfaces. For our films, < $100 \AA$ thick, the number of molecules per metal atom can be appreciable. For these fijms the coherence length of a Cooper pain in the transverse direction should essentially equal the film thickness, and therefore there shoulc be a good opportunity for "volume" superconducting electrons to interact effectively with strongly bound sunface molecules.

What we actually measure is the dc-critical current oi a film as a function of temperature, both before and after deposition of the chosen chemical. The critical current is taken to be that required to restore a small, fixed, resisiance lo a superconducting film (50). This is rot an ideal measurement for two reasons. Since the value of the resistance restored is very low, we are essentially locking at the very first portions of the film to be driven normal. This initial resistance will generally arise at flaws, unusually thin areas, or edges--the poorest portions of the film (51). 
Second, current-incuced transitions are plagued by the joule-heating winch begins when resistance develops (52). Newhouse (53) has shown that if a film element goes normal at relatively high currents, the joule heat generated can exceed a critical value and can drive adjacent areas nomal, an effect which can propagate as a thermal wave through the film. Such a process can start outside $\dot{O}_{\text {f }}$ the film area being studied--perhaps at the contact areas, which may be contaminated by the contact materià.

Howeven, these problems can at least be minimized. The effect of the edges is probably reduced because the films ane thin enough, and the temperature close enough to $T_{c}$, that the current is nearly uniformly distributed, instead of being concentrated nean the edges as would be the case with thick fiims and temperatures much. less than $\mathrm{T}_{C}(54)$. There is ro escaping the proplem of tLaws. However, by choosing always to restore a given resistance, we are presumably always driving the same element of film nommal (50). As shown in section III (Reslilts), the changes in $T_{C}$ produced by a deposited chemical are inaependent of the value of restored resistance as this value is varied by more tinan a factor of ten; this means that the effect of the chemical is the same over at least some small region of the film, and not merely at one flaw.

Choosing a smali value of restored reststance also minimizes the problem of thermai propagation. Resistance first appears at a flaw, thin region, or distribution of such. As Iong as the 
power generated, $I^{2} R$, is too small to cause a thermal wave, these regions will act independently of one another and the transition is called "isothermal." That is, the joule heating of a region already gone normal cannot raise the temperature of other regions, which will remain at the bath temperature $T_{0}$ and oxnibit critical currents chanacteristic of that temperature, $I_{C}\left(T_{0}\right)$. Since we typically wonk with a restored resistance $\sim 5 \times 10^{-3} \Omega$, and with critical currents $\lesssim 10 \mathrm{ma} .$, the power generated, $\lesssim 5 \mu \mathrm{W}$ is incieed Small.

The use of the geometry recommended by Newhouse (53), in which the contact areas are much wider than the film strig being studiec, eliminates the possibility of a thermal wave originating outside of the strip. Since the critical current increases with

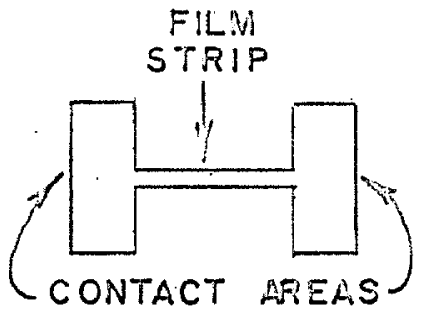

film width (53), the strip will become resistive while the wider dreas refidin superconduching. This ensures lhal conlaniraled regions at the contacts do not affect the measurements. For our experiments, the apparatus shown in Fig. I was constructed. It is essentially the union of an all-motal liquid helium dewar with an all-metal ultra-high vacuun (UHV) evaporation chamber; a detailed description is given in the next section. Ultra-iigh vacuum techniques are absolutely essential for experiments which are meant to study clean surfaces. Kinetic theory 


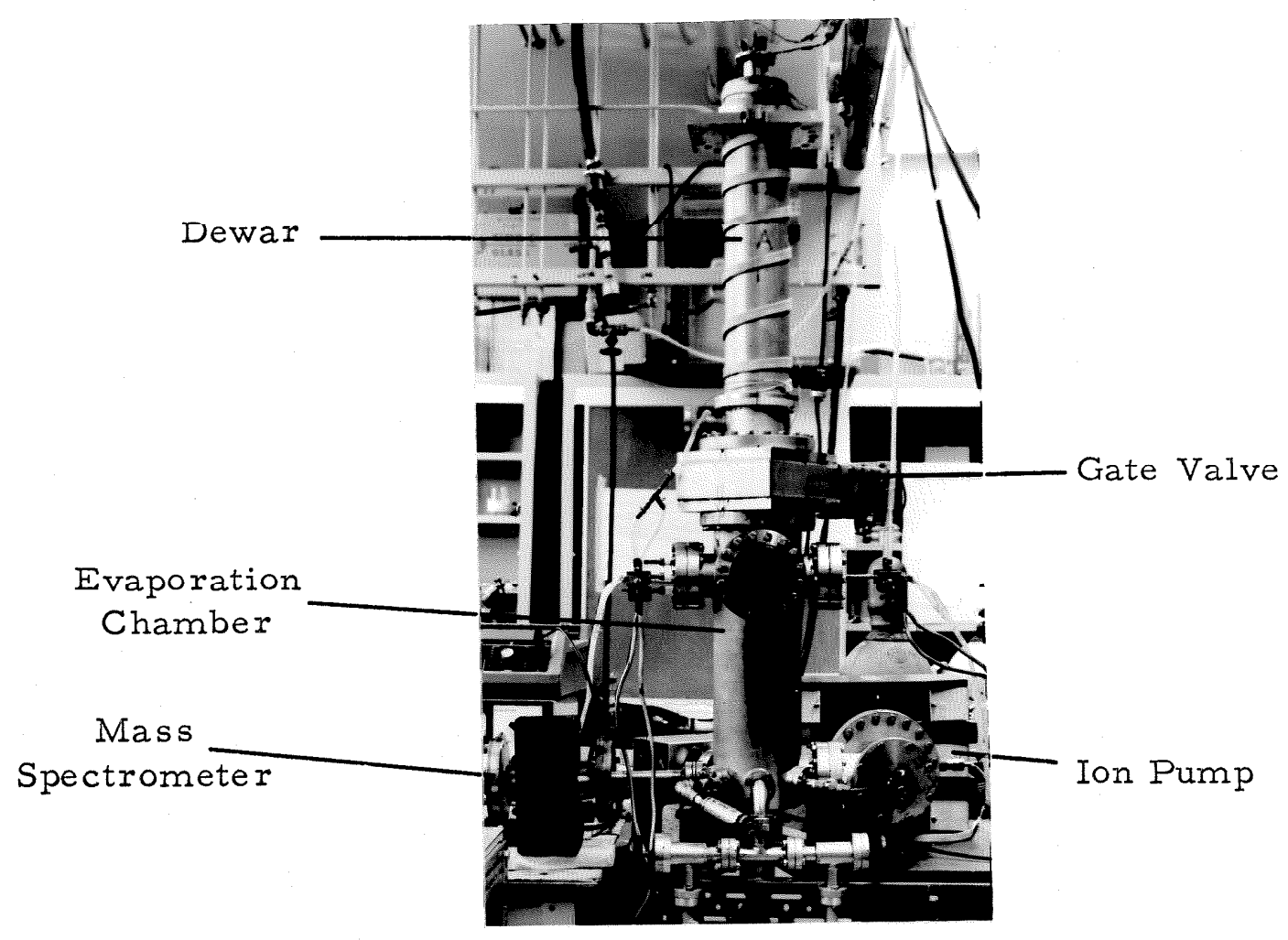

Fig. 1. Experimental Apparatus

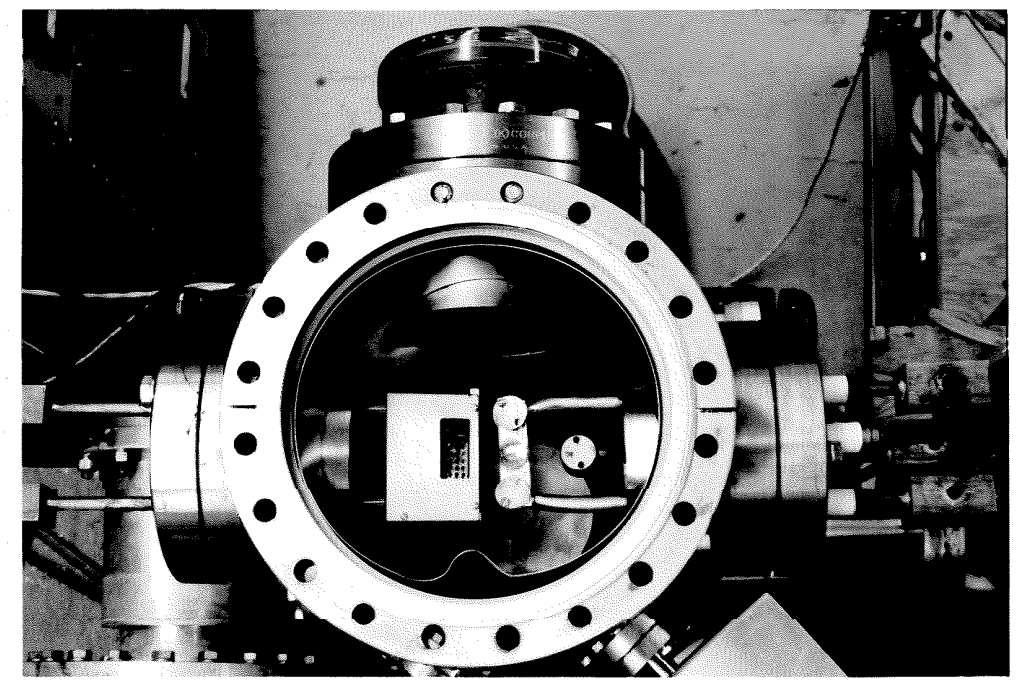

Fig. 2. Looking into evaporation chamber with evaporation sources in place 
calcuiations predict, and surface experiments verify, that at $\sim \mathrm{IC}^{-6}$ torn. a surface becomes covered with a monolayen of acisorbed gases in a matter of seconds (55). Since this time is inversely proportional to the effective pressure, it is obvious that the vacuum at a freshly deposited film must be $\sim 10^{-9}$ torn. or better in orcier to permit critical current measurements to be performed and a desired chemical deposited before surface contamination can occur.

It is unfortunately true, that the very nature of the experiment performed increases the difficulty of obtaining adequately low pressures. Finst, into a vacuum system carefully designed to eliminate all gassy structural materials, we must introduce an organic compound which by UHV standards has an enormous vapor pressure; anthracene has a vapor pressure of .I th at $35^{\circ} \mathrm{C}(56)$. Moreover, the compounc is usually a powder, with a large surface area for gas adsonption.

A second difficulty is a limitation on bakeout temperatures. It is standarc UHV procedume to bake a system unaer vacuum to $250^{\circ} \mathrm{C}$, if the elastomer Viton-A is present, and to $\sim 350^{\circ} \mathrm{C}$ or more if the system is entireiy metal (57). This is done to desorb gases from the walls, since the slow desorption of these gases would constitute the primary gas source in dn uribaked syslem. We are limited to $\sim 100^{\circ} \mathrm{C}$ for the system as a whole because of the necessity of using indium cryogenic vacuum seals in the dewar assembly. Indium molto at $156^{\circ} \mathrm{C}$, and at somowhat lower comporatures softens to the point that a seal can be ruptured by atmospheric pressure. 
Despite these obstacles, JHV can be obtained. In our expeniments, metal films are evaporatec in UHV onto a substrate in direct contact with liquid helium. Critical currents can be determined without exposing the filn surface to contamination. An appropriate chemical can then be deposited, and the propentics of netal fiim plus chemical overlayer can be cirectly compared to those of the film itseif. Tnis differential measurement of the effects of a chemical on a given film allows the relisile detection of effects much smallen than possible variations between films (58).
\end{abstract}




\section{Description of Apparatus}

The liquid helium dewar was designed by $A$. Kwiram and is descrihed in his thesis (59). The Drimary responsibility for the design of the evaporation and substnate systems belonged to D. D. Thomas; because of this, ard because apparatus not vastly different from ours is commercially available (60), an attempt will be made to discuss rainly those details of construction which are eithen unicue to oun apparatus or critical. to our particular experiments.

\section{A. GeneraI Description of Vacuum System (see Fig. 1)}

The evaporation chamber made by the ultek Corponation, is an upright stainless steel cylinder, $\theta^{\prime \prime}$ in diameter and $20 "$ tall, with ports fon acimiting the various vacuum components. AII static seals to the chamber are obtained by compressing annealec copper o-rings between stainless steel flanges. Fround the bottom is a ring of ports of various sizes to which are connected fore pumps, ion pump, thenmocouple gauge, ionization gauge, mass spectrometer, and up-to-atmosphere (UIA) valve. At other times the feedthroughs for a copper liquid nitrogen (IN) coil (Meissnen trap) were attachec here. A titanium sublimation pump is admitted through a port at the bottom of the chamber.

About two-thirds of the way up are three additional ports. Two opposed $3^{\prime \prime}$ ports admit the vacuum evaporators, one for tine experimental film and one for the chosen organic compound. A 
Pyrex window is sealed to the thind, $4^{\prime \prime}$ port. Figure 2 is a view into the chamber showing the evaporators in place and the port.

The chamber is capped by a $6^{\prime \prime}$ gate valve. Atop the gate valve, a reducing collar mates to the liquid helium dewar. A Viton o ring is uscd because the dewar was oniginally designed. with elastomer seals for EPR experiments. In addition, Viton o-rings are used with blank-off flanges on the ports through which the dewar had originally been evacuated. Compressed indium seals are used for the Iiquid helium temperature and UHV seals in the substrate assembly at the bottom of the helium well. When the gate valve is open, the vacuum chambers of the dewar are continuous with the evaporation chamber. If desired, the mass spectrometer tube can also be mounted on a port in the reducing collar, bringing it closer to the film surface.

The helium dewar was modified for this study by a brass substrate holder (SH) which bolts to the open bottom of the liquid helium well. The vacuum seal is made with compressed indium. A quartz substrate is sealed to the SH, forming the bottom of the helium. well. The ocal is again compressed indium. A brass compression flange ("presser-downer", PD) supplies the necessary pressure. An $0.005^{\prime \prime}$ stainless steel evaporation mask, cut to the desined form shape, fits onto the $P D$ and masks the substrate. Over the stainless steel mask is an appropriately cut I/I0" copper plate. Because of the thermal conductivity of copper, the mask acts as a helium temperature heat shield. Another helium temperature heat shield is bolted to the periphery of the substrate 
holder. A liquid nitrogen heat shield is boltec to the bottom of the LN well.

The substrate assembly is shown in cross-section in Fig. 3 . The heat shields, PD, and substrate holden are shown in Fig. 4. The llisk and a substrate with an evaporated film are shown in Fig. 5 .

B. Fumps

(a) Fore pumps.--The roughing system consists of two ultek somption pumps filled with tinde \#5X molenilar sieves. The pumps open to their common, I", tube into the chamber through separate ultek modeI \#40-131 I" right-angle vacuum valves. When cooled by IN they can rapidIy reciuce the system pressure to less than one micron $\mathrm{Hg}$. The pumps are regenenated by jaking them at hign temperatures to dnive off adsorbed gases.

The advantage of sorpticn purps over mechanical forepumps is their cleanliness. It is difficult to prevent backstreaming of pump oil from a mechanical pump. Since our experiments are designed to detect the effects of organic chemicals on clean film surfaces, the introduction of appreciable amounts of pump oil into the system would be intolerable.

The chief disadvantage of sorption pumps is their finite capacity. If the system is fumped down nore than once without the sorption pumps having been warmed, they can reach saturation and adequate roughing pressures can no Iongen be obtained. This is particularly likely if the pumps have pumped on a leak duning 


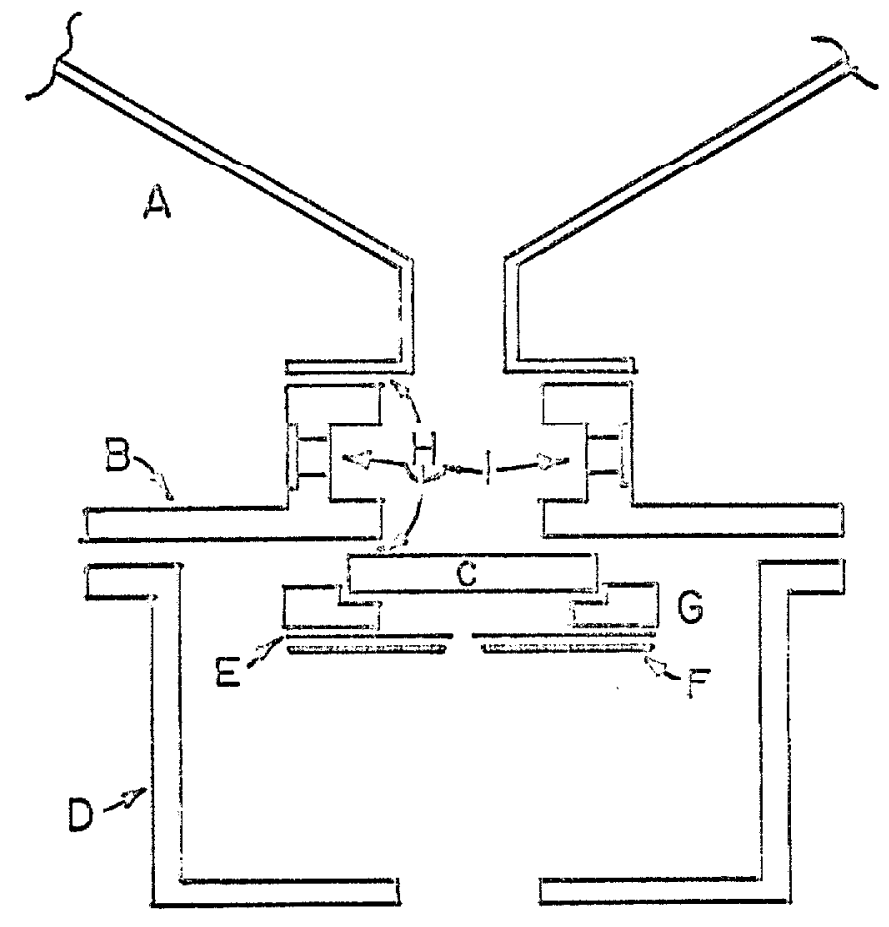

Fig. 3. Schematic Cross-Section of Substrate Assembly (approximately actual size). (A) Dewar helium well; (B) Substrate holder; (C) Substrate; (D) Heat shield; (E) Evaporation Mask; (F) Copper Over-mask; (G) Compression Flange; (H) Indium cryogenic vacuum seals; (I) Feed-through wells. 


\section{1}

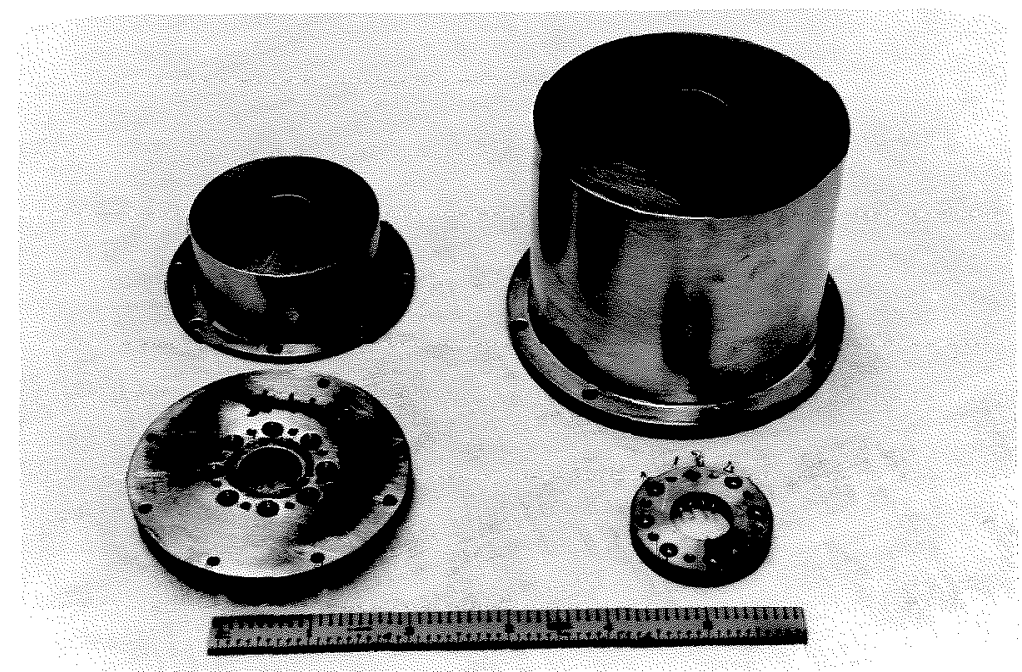

Fig. 4. Back, left: helium heat shield; right: LN heat shield Front, left: substrate holder; right: compression flange

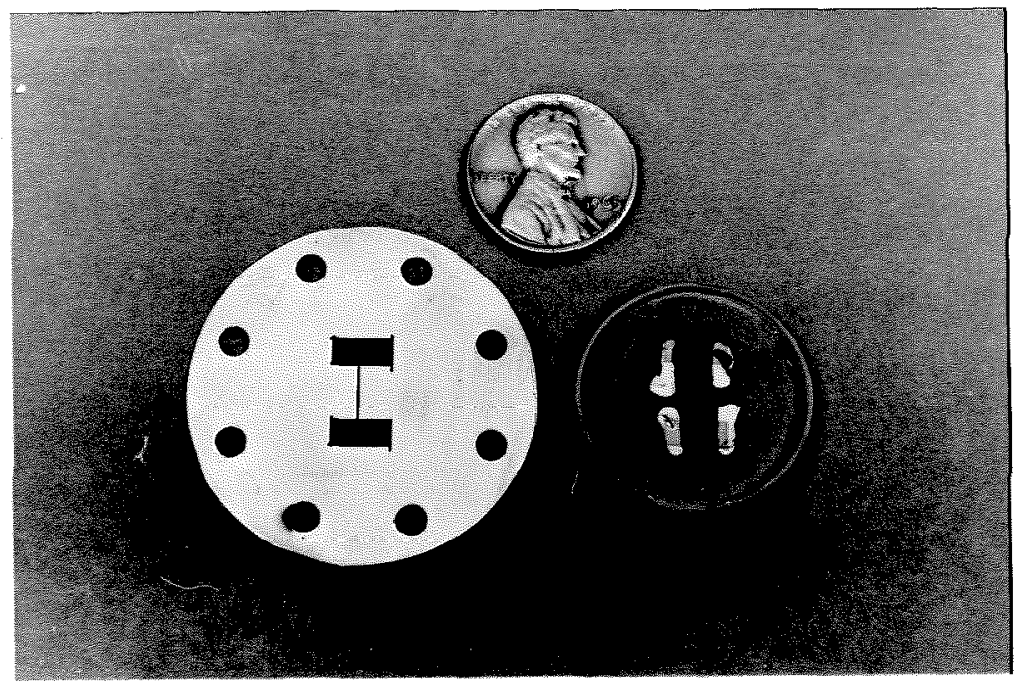

Fig. 5. Evaporation mask and substrate with evaporated film 
the first pump-down. Such a Eailune of the sorption pumps recessitates either an appreciable delay wnile they are baked out, or else the "exasperation" method of attaching a mechanical forepump through the UTA valve.

(b) The primary pump is a liltek lool/s tilánium yelier-ion pump. It is connected to the evaporation chamber by an ultek $3^{\prime \prime}$ right angle vacuum valve (\#40-31I). The principles and ac̀vantages of ion pumping are explaincd clocwhorc (57) and wilz not bo repeated here.

An important disadvantage for the present application is that the getter-ion pump can be contaminated hy high molecular weight onganic vapors such as Denzene, toluene and any of the organic compounds with which we have experimented (57). The cathodes build up a carbonaceous deposit which is difficult to sputter. These areas do not pump efficiently and therefore, the pumping speed is decreased. Such contamination has required that the ion pump be chemically cleaned three times in a year and a half. The principal problem seems to be that occasionally some of the experimental compound sublimes duning bakeouts and ends up in the pump. However, the high vapor pressures of these compounds means that they must be continuously evolving smali amounts of vapor which enter the pump, causing eventual contamination.

The contamination problem, incidentally, cancels one apparent advantage of ion-pumps. In principle, the pump current should be a measure of the pressure; the ion pump itself should therefore eliminate the necessity of an ionization gauge. However, an ion 
pump has some leakage current which is incependent of pressure and which creates a low-pressure limit (usualiy $\sim 10^{-9}$ tomr.) to the pump's usefulness as a gauge. Contamination can increase the leakage current to such an extent that despite a true system pressure of $<10^{-9}$ torr, the pump will indicate as nigh at $10^{-7}$ torr, thus making an independent pressure measurement essential. Another disadvantage of ion pumps is the reluctance to pump large quantities of gas. This is important during initial pumpdown and during pressure surges in the course of a metal-film evaporation.

(c) The system also contains a titanium suhlimation pump (ISP), consisting of a Eilament holder containing four titanium filaments. A stainless steel shield is mounted atop the holder to prevent titanium from being sublimed onto the vacuum evaporators or substrate. The assembly is admitted through a port in the bottom of the evaporation chamber. Fresh titanium surfaces, thermally sublimed onto the chamber wall from the TSP filaments, pump neutral reactive gases by combining with them to form stable compounds. Such surtaces have pumping speeds of the order of $10 \mathrm{~L} / \mathrm{s} /$ in. $^{2}$ at $20^{\circ} \mathrm{C}$, and are especiaily effective in combination with an ion pump (61).

This additional purping speed is inporlan al relatively high pressures; it greatly decreasec pumpdown town from roughing pressure $(\sim 1 \mu)$ to $\sim 10^{-7}$ torn, at which pressure leak testing is done and bake out is begun. The ISP is most important, however, during vanadium evaporation. Often lange quantities of gas are 
evolved which the ion pump alone cannot handle without momentary, but disastrous increase in system pressure from $\sim 5 \times 10^{-10}$ torr. to $\sim 10^{-7}$ torr. The TSP limits the pressure rise to less than $3 \times 10^{-8}$ torr. and decreases the time during which it obtains. Unfortunately, the design of the craporation chambcr limito the efficiency of the TSP. It is narrow, and as a consequence, the walls get quite hot during titanium sublimation. The pumping efficiency of a titanium film decreases with increasing temperature. One solution to this problem is cooling coils brazed to the chamber's exterior. This was not done in construction, and is not feasible now that the flanges are attached.

An even more efficient solution is to sublime the titanium onto a IN coil (Meissner trap), combining titanium gettering with IN cryopumping. However, the narrow chambers cannot fit both the TSP and Meissner trap. We had in fact installed a Meissner trap prior to the addition of the TSP. The IN cryopunping was capable of maintaining acceptably low pressures during evaporations, but the coil was difficult to clean in place, and could not be readily renoved because it was difficult to make vacum-tight comnections inside the chamber.

During pumpdown, the reduced efficiency of the TSP is accepted; the only countermeasure taken is a small fan. During an actual experiment, maintenance of the ultimate pressure is critical. Therefore the evaporation chamber is surrounded by a styrofoam jacket filled with dry ice. 
C. Pressure Measurement

(a) Roughing pressures are reasured by an NRC thermocouple gauge and box. This gauge is isolated from the system by closing a Whitey \#IGM4 poppet valve after the pressure is below I micron. The UTA valve is also a Whitey \#IGM4 valve.

(D) Pressures below i micron are measured by a hot-fizament Bayard-Alpert ionization gauge (Ultek model \#60-722). The tube has a glass envelope and is extemally mounted on a $I I / 2 "$ flange by a tube $2^{\prime \prime} \times 3 / 4^{\prime \prime}$. Because of the relatively low conductance of this tube, it is likely that at indicated gauge pressures of $\sim 5 \times 10^{-10}$ torr. or better, the gauge tube may have the highest pressure in the system. As a result, pressures are generally quoted without a gauge correction which is in principle calculable using the mass spectrometer, and was estimated to be $\sim 1.4$ on one occasion.

(c) An Associated Electronics Industries model MS-10 mass spectrometer is also incorporated in the vacuum system. This is an $180^{\circ}$ magnetic deflection instrument. It has $\mathrm{m} / \mathrm{e}$ ranges of 2 , $3,4,12-45$ and $18-100$ using an $0.020 "$ siti. With this slit we can easily resolve peaks one mass unit apart below $\mathrm{m} / \mathrm{e} \sim 80$; the stated detection limits are $6 \times 10^{-12}$ torr. for $\mathrm{N}_{2}$ and $\sim 10^{-11}$ torr. for $\mathrm{O}_{2}$. The otainlcoo otccl (SS) analyzer tube is connected to the evaporation chamber by an SS tube $6 " \times 1 "$.

The mass spectrometer was addec to the system after we discovered that oxygen deposited on the surface of a vanadium film can increase its $T_{C}$ (see section III, Results, $A$ ). Witnout knowing 
the composition of the gases released during a chemical deposition, it is not possible to unambiguously attribute an effect to the selected chemical, and not to some other evolved gas.

We found that in general, hydrogen was the gas responsible for most of the pressure increase during evaporation of the chemical overlayer. Hydrogen has been seen to be the principle gas during experiments in which $T_{C}$ was increased, and also in others during which it was decreased. The hydrogen presumably is dissolved by the tantalum boat which holds the chemical, and is released by heating.

The influence of oxygon oun safely be discounted since its partial pressure during a chemical evaporation may only rise from $\sim 10^{-11}$ torr. to $<5 \times 10^{-10}$ torn., while the system pressure is increasing from $\sim 5 \times 10^{-10}$ torr. to $\sim 3 \times 70^{-9}$ tomr, die to the release of hydrogen. Other gases, such as $\mathrm{CO}_{2}(\mathrm{~m} / \mathrm{e}=44)$ and $\mathrm{N}_{2}$ or $\mathrm{CO}$ (both $\mathrm{m} / \mathrm{e}=28$ ), have been monitored during organic chemical evaporations, and have only displayed small variations.

Hydrogen is also the principal gas released during vanadium evaporations and during titanium subimation from TSP.

The mass spectrometer performs two other valuable functions. First, Dy giving the residual gas spectrum it serves as an indicator of general system cleaniiness. In particular, the dewar had originally been used for EPR experiments in a diffusion-pumped system and was found to be contaminated, as indicated by tine presence of high mass peaks $(\mathrm{m} / \mathrm{e}>45)$. After chemical cleaning of the dewar and successive experiments involving cleaning of the 
evaponation chamber plus overall system bakeouts, peaks of $\mathrm{m} / \mathrm{e}$ $>45$ almost completely disappeared. The cleanliness of the system is indicated by the fact that, often, most of the residual gas is water, carbon monoxide and carbon dioxide--the vapors evolved by Viton A (62). In one, rather clean, experiment these three gases comprised $\sim 82 \%$ of the residual gases; hydrogen, argon and helium comprised $\sim 9 \%$. Methane, which is synthesized in the ion pump from water and carbon dioxide, made up most of the remainder. Second, and of supreme practical importance, is the ability of the mass spectrometer to function as a leak detector. Ion pumps are extremely efficient at romoving ractive gasce. Thorcfore, at low pressures with no leaks there is almost no peak at $\mathrm{m} / \mathrm{e}=32$ (oxygen). The presence of an appreciable peak at this mass is therefore characteristic of a leak. By the use of the mass spectrometer and a probe gas (usually helium), it has been possible to pinpoint, and occasionally correct, leaks at pressures as low as $\sim 2 \times 10^{-9}$ torr.

\section{Vacuum Evaporation System}

(a) Evaporation sources.--Helical tungsten coils are used as vanadium evaporation sources. Since vanadium alloys with tungsten, the coils eventually fail. We could not reliably evaporate vanadium from even the thickest commercially available sources, made trom $s \times$. UsU" braided tungsten wire (R. D. Mathis, \#BI-3 $\times 0.030 \mathrm{~W})$, and several experiments were ruined because of such coil failures. 
Home-made coils were therefore wound to approximately the same dimensions, from $4 \times 0.30^{\prime \prime} \mathrm{W}$. No experiment has failed (completely) because of a failure of such a coil. Nevertheless, a second coil is often mounted on the metal evaporator, as insurance.

Indium and tin are evaporated from tungsten or molybajenum. dimple boats (Mathis, $\mathrm{VE} 4, .005^{\prime \prime} \mathrm{W}$ or Mo).

The chemical overlayer is evaporated from a covered tantaIum boat (Mathis, \#NE-2, cover \#ME-2B). The boat is two to three times as wide as the metal sounce to ensure that the metal filn can still be completely covered, despite minor misäignments of the two sources.

(b) Source supports.--Separate flanges, each with watercouled OFHC copper suppurls brazed lo the hollow netal-ceramic vacuum electrical feed-throughs (Ceramaseal Corporation \#804B2779-2) mounted on them, carry the two evaporation sources. Figure 6 shows the two flarges with sounces in place. Watercooling the mount for the metal source is important because of the high temperatures $\left(\sim 2000^{\circ} \mathrm{C}\right)$ necessary to evaporate vanadium. Three supports are presently mounted on this flange, capable of mounting three metal sources.

The flange for the organic chemical evaporator mounts only one source. This sounce has been completely enclosed by copper heat shields, apart from a I" $\times I / 2 "$ hole for evaporation. The water cooling and the heat shields are necessary to prevent the source from being heated during bakeouts or metal evaporations; 


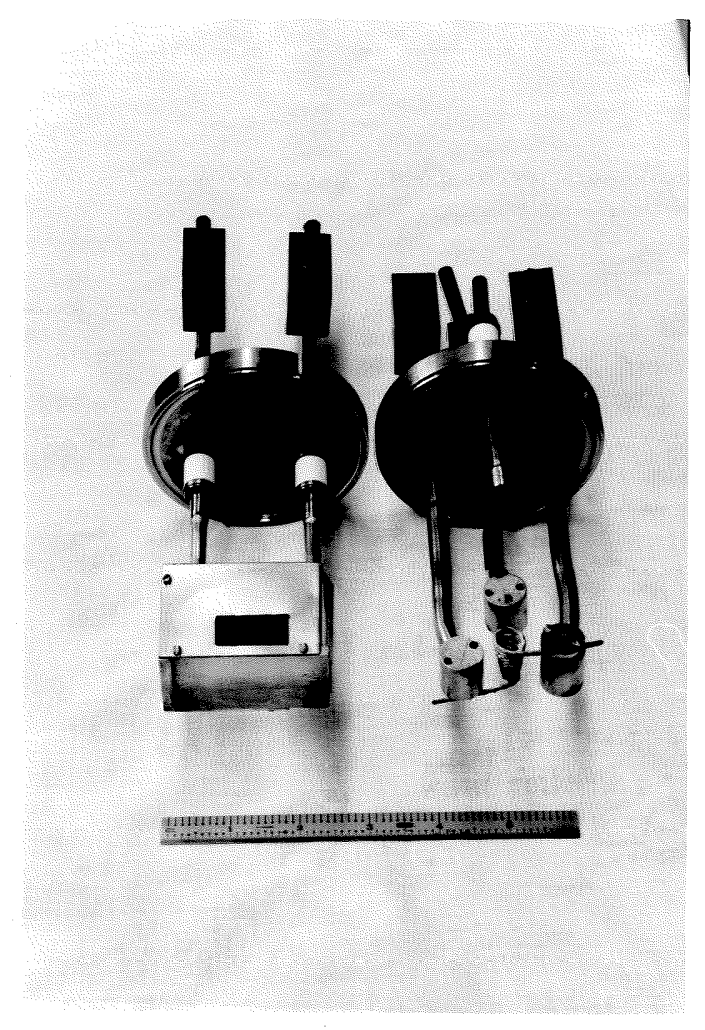

Fig. 6. Vacuum Evaporators, Left, organic chemical; right, metal evaporator 
such heating can cause the chemical to sublime prematurely, contaminating the system.

Because the problem of contamination during bakeouts was not anticipated, the present organic evaporator was originaliy used for the metal source; the original organic evaporator was not water-cooled, but merely had solid supports. The change was effected because cooling the organic evaporator flange during bakeouts by a stream of air proved marginally effective, at best. At worst, it once proved disastrous when the fan burned out, the organic sounce heated up and the entire system was contaminated by chionanit, the chemical in the hoat.

(c) Current source.--The currents necessary for the resistance-heated sources come from a 120 volt line, using a step-down transformer controlled by Variacs. The source for the metal evaporator is a 115/11 volt transformer and a $10 \mathrm{amp}$. Variac. Ihat tor the organic evaporator is a $113 / 5$ volt transformer and 5 amp. Variac.

E. Chemicals

Vanadium used in early experiments (Table I, \#5; II, \#I; III) was nominally $99.8 \%$ pure. Spectroscopic analysis revealed the following impurities: Cu, 1 ppm; Cr, 47 ppm; Fe, 344 ppm; Mg, $25 \mathrm{ppm}$; Mn, $178 \mathrm{ppm}$; Si, $1400 \mathrm{ppm}$. All other vanadium experiments were done with zone refined, nominally $99.999 \%$ pure vanadium from Research Inorganic Chemicals. Spectroscopic analysis could detect no impurities. Film properties for the two purities are essentially the same. 
The indium experiments were done with nominally $99.999 \%$ pure metal. Spectroscopic analysis revealed less than $5 \mathrm{ppm}$ copper.

The organic compounds used were either zone refined (anthracene) or recrystallized and then sublimed, usually two or more times. Paramagnetic impurities were not always removed by this procedure. They were sometimes found in very low concentration, but in both "enhancers," molecules which incrcascd T, and "depressors," those which decreased it.

In the experiment involving the deposition of oxygen, the oxygen was generated in the vacuum chamben by the thenmal deromposition of silver oxide.

\section{F. Substrate Assembly}

(a) A cross-section of the substrate assembly has been shown in Figure 3, and the various components pictured in Figures 4, and 5. The substrate holder is machined from brass, and the only critical feature is the mounting of the vacuum electrical feed-throughs, to be discussed below. The PD is also machined from brass.

The two heat shields are machined OFHC copper cans. They prevent room temperature radiation from reaching the film, except in the small solid angle through which the chemicals are evaporated. The small aperture in the heat shields should serve an additional function. Since the standard kinetic theory calculation for the number of molecules striking a plane surface involves 
integrations over the full solid angle of $2 \pi$ at the surface, at any given system pressure the shields should reduce the rate of surface contamination by a factor of 5 or 10 .

(b) Masks.--The geometry of evaporated films is determined by the pattern cut in a $0.005^{\prime \prime}$ stainless steel mask, located I/8" from the substrate. Two different four-point probe geometries have been used. The initial pattern

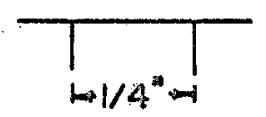

was soon changed to the "H" pattern recommended by Newhouse (53):

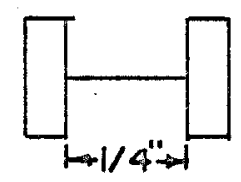

The defining slit width has heen $0.010 "$ and $0.006 "$.

(c) Substrates are optically flat, $y$-axis cut singlecrystal quartz disks, $I^{\prime \prime}$ in diameter $\times 1 / 8^{\prime \prime}$ thick, from Valpey Corporation. At helium temperatures, single crystal quartz has a thermal conductivity in the y direction slightly greater than that of copper (63). This makes it the best available substrate material, since thermal gradients across the substrate must be minimized.

(d) Film leads.--In order to make electrical contact to a film, four islands of electrically conducting paint are put onto the substrate before it is mounted on the SH. Silver paint is spread with a thin metal wire, and care is taken to make the edges toward the film very thin and smooth. Although the paint 
is initially air-dried, it then sits in vacuum for a minimum of four days in the evaporation system. This includes $\sim 48$ livur's of being vacuum baked to $\sim 90^{\circ} \mathrm{C}$ during the system bakeout.

Superconducting $\mathrm{Pb}$ contacts have also been used. These are precvaporatcd onto a clcan quartz dish at $-10^{-5}$ torr. in a separate evaporation chamber. They are defined by an $0.005^{\prime \prime}$ stainless steel mask held flush with the quartz surface. This prevents lead atoms from "bouncing" onto the area to be used for the experimental film.

Although there have been literature reports that silver paint contacts can contaminate evaporated metal films (64), we have found that the lead contacts produced no significant differences. This probably results trom the fact that the portion. of a fiim whose properties are being studied is quite far removec from the contacts: The vacuum bakeout may also contribute significanlly lu lie benign behavior of our silver paint contacto.

Connections to the silver paint contact is lands are made using \#40 nyclad copper wire stripped of its insulation by Strip-X and tinned with In-Sn solder. The wires are soldered to four binding posts of thicker wire epoxied (Resinweld) into radial holes in the PD. They are attached to the contacts by "gluing" them down with drops of the same silver paint used for the contacts. No experimental failures have ever been traced to a wire pulling away from its contact.

Wires from the binding posts of the PD are soldered to four heavy wires epoxied to the substrate holder. From these binding 
posts, wires are soldered to the four glass-metal vacuum electrical feedthroughs (Glass Instruments Inc. \#1207) mounted in the substrate holder. These carry the electrical leads from the vacuum chamber into the helium well.

Inside the helium well wires from the feedthroughs terminate in a modified transistor socket. Since the helium well is pumped to as low as several torr., the wires from the mating socket go through a simple vacuum seal in the cap.

(e) Cryogenic, vacuum-electnical feedthroughs.--Bringing the electrical leads into the vacuum through the liquid helium provonto thom from acting as heat leaks. This procedure is necessary because we assume the film temperature to equal that of the helium bath, although the film is not immersed in helium. Inefficient thermal grounding of the leads could raise the film temperature. Unfortunately, these feedthroughs are probably the most critical vacuum element in the entire apparatus. Repeated thermal cycling between room and helium temperatures can cause them to fail, presumably because of fatiguing due to differential thermal expansion.

However, the previous set of feedthroughs was completely leak-free for many months--many helium transfers and even more cyclings between I and II--before they eventually failed. 'Ihe replacement has performed similarly well for several experiments, and should last as long. The critical features in mounting these Ieedthroughs seem to be avoiding thermal shock during the soldering process and keeping solder out of the hole through the $\mathrm{SH}$ in 
which the feedthrough sits. The latter is made difficult because of the small clearances dictated by the size of the feedthrough and the maximum possible size of a transverse hole in the substrate holder. Capiliary action tends to draw liquid solder into the narrow annulus between teedthrough and well. An attempt is made to minimize this by counterboring the well for the Iip of the feedthrough.

HELIUM

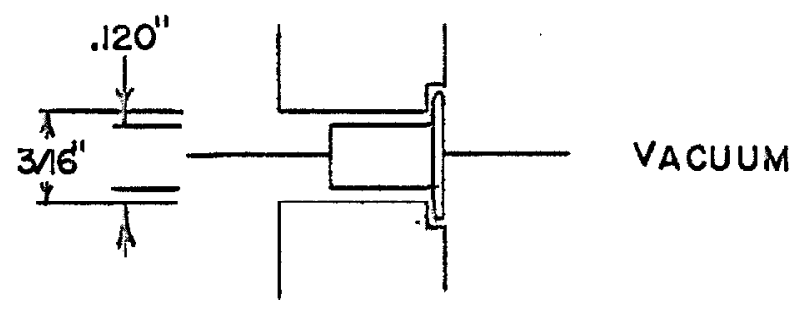

The procedure for soldering the feedthroughs is as follows. They are placed in their wells in the SH and are held in place by the tension of a cylindrical brass strip which pressed against the wire of the feedthrough. This assembly is then placed in an oven and heated slowly to a temperature above the melting point of soft solder. It is then removed and placed on a hotplate to maintain the necessary temperature. A minimal amount of solder is then applied, using lloyd's stainless steel soldering flux. Turning down the hotplate temperature allows the assembly to cool s lowly.

\section{G. Critical Current Measurement}

The four leads brought oul of blie heliun well are connected by a shielded cable to a simple apparatus designed to measure the voltage drop across two voltage contacts of an "H"-shaped film as 
a function of an impressed current supplied through a separate set of two current contacts. This well-known four-point probe technique allows the voltage-drop to be measured in a circuit which carries no current. Thus, contact resistances do not affect the measurement.

The circuit diagram for the apparatus is given by Figure 7. The resistance of a film can vary from 0 ohms, when it is superconducting, to more than 10,000 ohms, when fully normal; the source is of moderately high impedance, and can maintain an approximately constant current as a superconducting film goes normal. (A rigorously constant current source is not necessary.) The current through the film is determined by the voltage drop across a $I K$ onm resistor. This current-medsuriny. vollaye is displayed on the $y$-axis of a Moseley $x-y$ recorder. The voltage developed across the film is directly displayed on the $x$-axis of the recorder. The maximum inotrumental ocnoitivity, $0.1 \mathrm{mv} / \mathrm{inch}$, is adequate without prior amplification. Thus, the primary experimental data are curves displaying the voltage developed across the film as a function of impressed curnent. (See Fig. 8.)

\section{H. Tiquid Helium Temperature}

Helium is transferred with a Supairco transfer tube. Helium levels in the dewar are determined by three carbon resistors suspended at different heights in the dewar. We use $300 \mathrm{ohm}$ Allen-Bradley resistors with the porcelain coat ground off. When $\sim 9 \mathrm{~V}$ is put across such a resistor the current drawn will change 


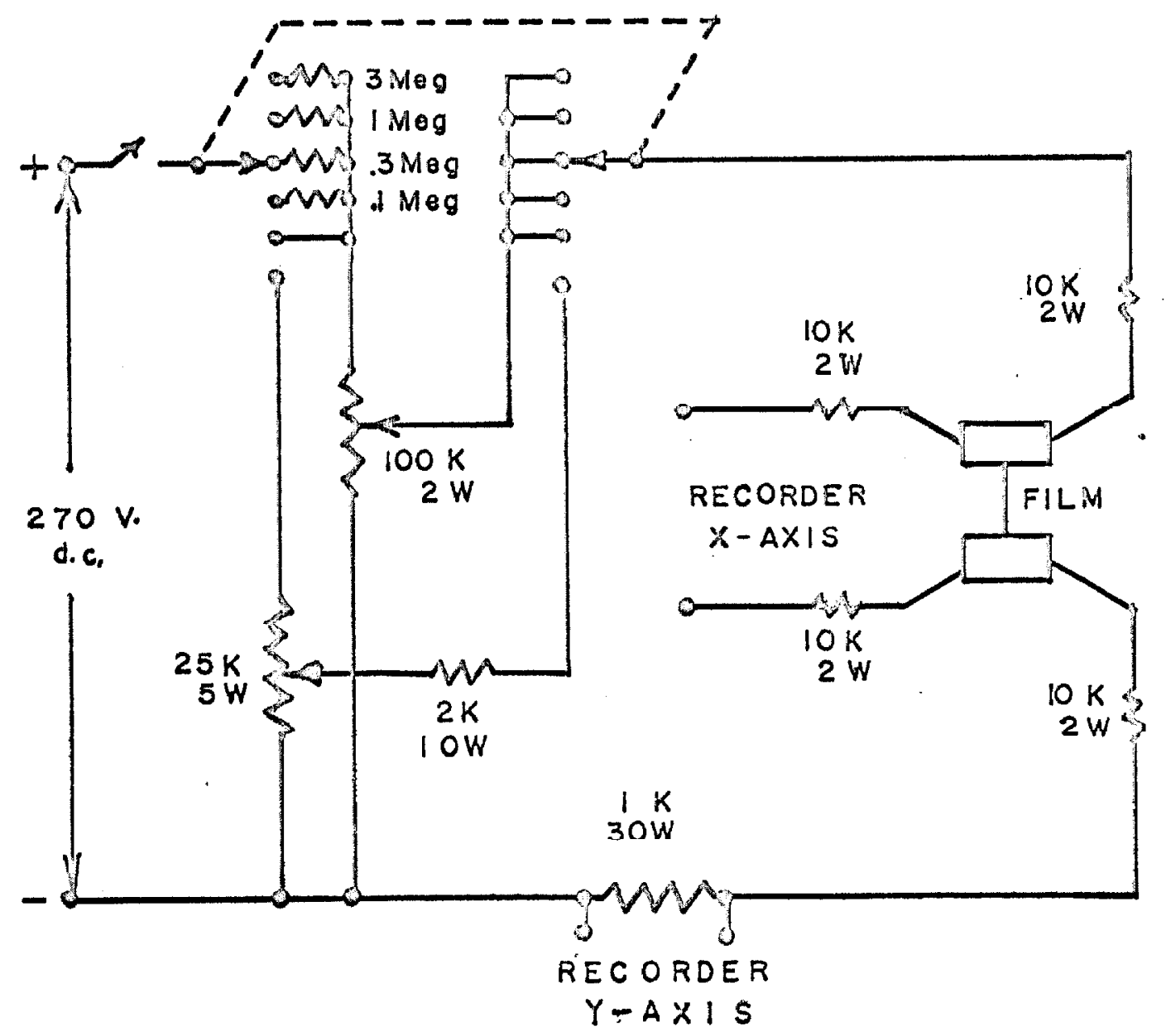

Fig. 7. Critical Current Measuring Circuit 
from $\sim 8 \mathrm{ma}$ to $\sim 4 \mathrm{ma}$ as the liquid helium level rises past it. Wires to the resistors are simply epoxied into a hole in the helium well cap.

Temperatures are determined from the vapon pressure of the pumped helium. The pressures are measured with a precision momometer via a tube which terminates near the substrate. Except for the actual measurement of $\mathrm{T}_{\mathrm{C}}$, experiments are usually done entirely below lne $\lambda$-poinl in order to eliminate thexmal gradients in the Iiquid helium. These precautions are taken to ensure that the temperature at the helium side of the quartz substrate should be very close to the temperature derived from the helium vapor pressure curve. Thermal gradients across the substrate caused by room temperature radiation should be quite smail because of the high thermal conductivity of the quartz at these temperatures and because of the shielding provided by one liquid nitrogen and two liquid helium temperature heat shields. The effectiveness of this arrangement is demonstrated by the fact that films heid at $\sim 1.8^{\circ} \mathrm{K}$ (indicated), later shown to have transition temperatures only $0.2^{\circ} \mathrm{K}$ higher, were superconducting during the latter part of the metal evaporation, while hot metal atoms were striking the surface, and the source at approximately $2000^{\circ} \mathrm{K}$ was only ten Inches away.

The helium vapor pressure is regulated by a diaphragm manostat built by A. I. Kwiram (59) with a more flexible diaphragm installed by D. D. Thomas. This manostat seems to function bettor as the vapor pressure is decreased and the helium is more nearly 
continuously pumped. Pressure stability is quite acceptable by $350 \mathrm{~mm} ., \sim 3.5^{\circ} \mathrm{K}$. Below $250 \mathrm{~mm} .\left(3.2^{\circ} \mathrm{K}\right)$, the pressures can be kept steady to within 0.1 on 0.2 torr., the reading accuracy of the manometer, during a measurement. 


\section{Procedure}

Our experimental procedure is as follows:

(a) The system is thoroughly cleaned of metal and organic compounds from the previous experiment. The evaporation chamber and gate valve are wiped with methanol-soaked Kimwipes. Deposits which have flaked off the chamber walls are removed with a vacuum cleaner. Coppen pieces--evaporators, heat shields and over-mask-are polished with steel wool and/or dipped in acid. Nitric acid may be used to remove vanadium deposits, but usually hydrochloric acid is used to remove, copper oxide. To ensure that no grease, "finger juice," or errant chemical from the previous experiment is returned to the clean system, all components, however cleaned, are given a final methanol rinse.

Because it is tedious and time-consuming to clean the evaporation chamber and gate-valve, removable copper shields have recently been made. They largely protect these areas during depositions, and can be easily cleaned upon removal. Despite the fact that these shields triple the number of surfaces which must be degassed, they still effect a net savings in effort and time, with no apparent sacrifice in ultimate pressure.

The surface of the vanadium chunks used for cvaporation is cleaned by etching in dilute nitric acid.

Tungsten coils and "dimple" boats are cleaned either by following the directions in Gutnrie's book (57h), on hy using sodium peroxide. 
The organic sounce is simply boiled several times in the appropriate solvent to remove the previous chemical.

A dirty quartz substrate is cleaned as follows:

(i) An organic solvent (usually trichloroethylene) is used to remove most of the dried silver paint and previous organic material.

(ii) Concentrated nitric acid removes the metal films. (Sometimes aqua regia is used and step ( $i$ ) is eliminated.)

(iii) The above two steps do not generally remove all traces of the previous experiment. The quartz disk is next rubbed gently between clean fingers, using Lava soap ds a cledring ayenh. Careful sludies hy elechrom microscopy and other techniques nave verified that this simple procedure is better than such extensive techniques as ultrasonic cleaning etc. (65) (which we couldn't do anyway).

(iv) The disk is next rinsed in tap water ard then rinsed in distilled water for several minutes.

(v) A methanol rinse completes the procedure. The disk is allowed to ain dry.

(b) The evaporation chamber is then reassembled with a new metal source, a new (organic) chemical, and new titanium fizaments for the TSP, if necessary. The dewar, with the clean substrate mounted in the substrate assembly, is placed atop the reducing collar, closing the vacuum system. 
(c) The system is then evacuated to $\lesssim I$ micron. At this time the TSP is degassed, as recommended by UItek (60). The forepumps are then valved out and the ion pump plus TSP reciuce the pressure to $<10^{-7}$ torr. A preliminary leak test is then made, using the mass spectrometer with helium as a probe gas. It is not unusual at tris point to find a flange that has to be further tightened.

(d) After leaks have veen correcled, he system is baked to $\sim 100^{\circ} \mathrm{C}$. The evaporation chamber is usually heated by infrared lamps, although sometimes heating tapes are used; the dewar io heated by tapco. Infrarcd lampo aro alwaye uecd for heating the odd places on which it is impossible to put a tape. The mass spectrometer and ion pump have their own heaters.

The bakeout is usually continued for about 48 hours, or until the pressure while hot is $\sim 10^{-6}$ to $10^{-7}$ torr. During this time the vanadium is given a preIiminary degassing.

(e) The bakeout is stopped and, as the system cools, the pressure falls to less than a few times $10^{-9}$ torr.--usually to $<6 \times 10^{-10}$ torr. The system is again leak tested to verify that no leaks have developed during bakeout. At some time during the finai pumpdown, the vanadium and organic chemicai are degassed, and small amounts of both are evaporated. The surface of the substrate is protected by closing the gate vaive.

(f) When a satisfactory base pressure has been achieved, the liquid nitrogen weil of the dewar is Iillea. Inis usually done the evening before an experiment is to be run, to allow the 
helium well and substrate assembiy to slowly cool overnight. The liquid nitrogen level is maintained by a Harvey Control Co. level controller. The helium well is kept at a small positive pressure of helium gas during this period to prevent the condensation of water vapor or liquid oxygen.

(g) The actual experiment begins with the transfer of liquid helium into the helium well of the dewar. The helium temperature is then usually reduced to $-1.8^{\circ} \mathrm{K}$, well jelow the $\lambda$-point at $2.17^{\circ} \mathrm{K}$. Both metal and organic films are evaporated through the fixed helium temperature mask onto the quartz substrate in dinect contact with supenfluid helium. Pressures during vanadium evaporations are $<3 \times 10^{-8}$ torr., during the indiur. and organic evaporations are $<3 \times 10^{-9}$ torr. and are generally $<6 \times 10^{-10}$ torr. at ali other times.

Metal is usually evaporated until the film superconducts. However, the existence of an approximate relationship between the resistance of a vanadium film, freshly depositec on a helium surface, and its transition temperature (Fig. 12) ailows us to evaporate to a predetermined resistance while holding the temperature somewhat above the expected $T_{c}$. The critical curnent, I ${ }_{c}$ is then measured as a function of temperature over some limited range below the $\lambda$-point. The critical current at a particular temperature is taken to be that current necessary to restore a certain fixed resistance to the film, generally between $10^{-3}$ and $10^{-2}$ onms. Figure 8 shows a typical current-vollaye curve; $I_{c}$ can be taken as, that current which restores any given value of 


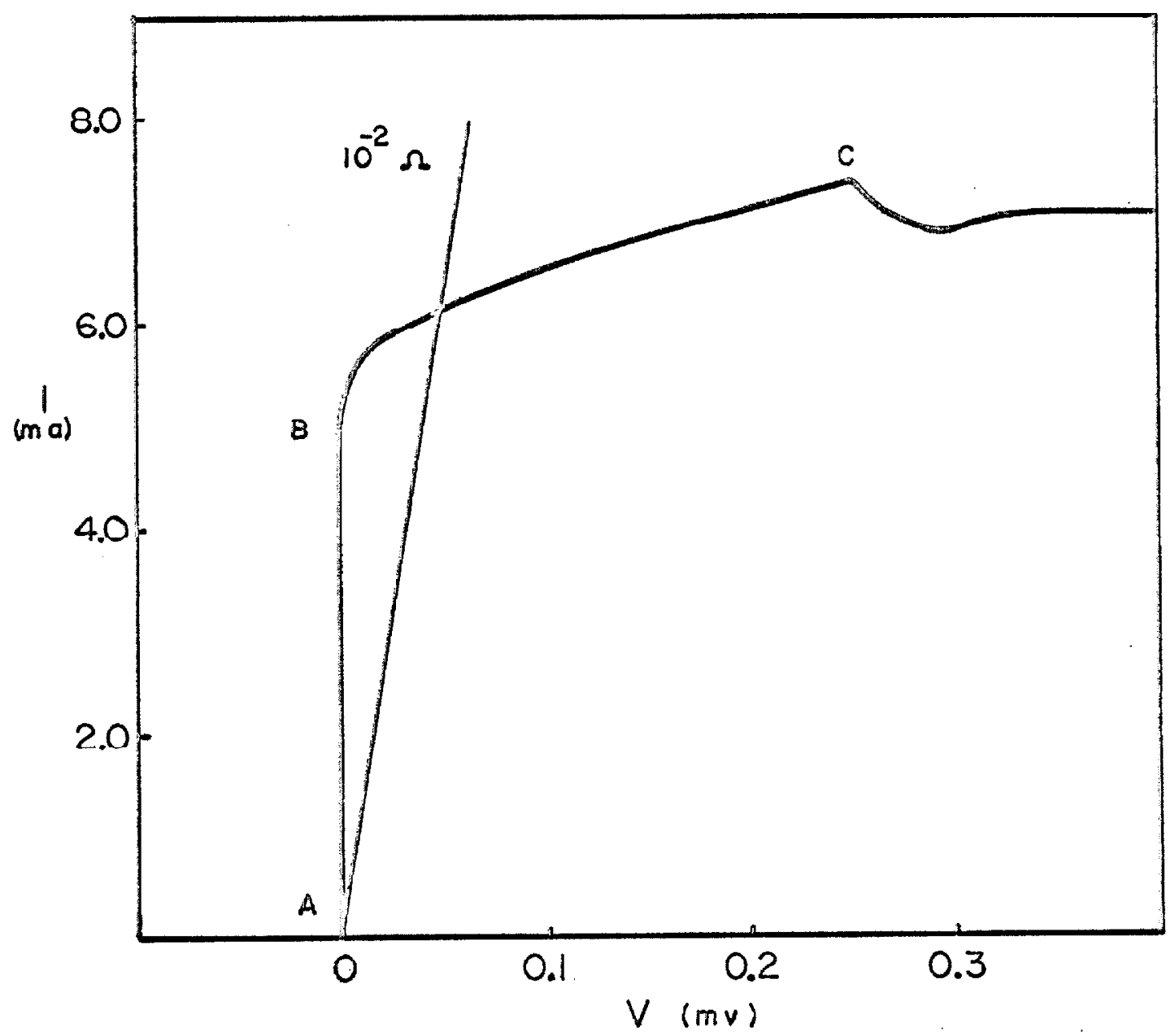

Fig. 8. Typical current-voltage curve for a superconducting vanadium film. Region $A-B$, the film has zero resistance. $B-C$ is a region of "isothermal" transition to the normal state. Point $C$, the film is driven normal by a propagating thermal wave. At the intersection with the straight line the restored resistance is $10^{-2}$ 
resistance within the "isothermal" region. $I_{c}$ at a particular Lemperdlure does not change with time at a system pressure of $\sim 6 \times 10^{-6}$ torr. as it would if surface contamination by residual gases were important (66). Nevertheless, to ensure that the surface remains clean, the measurements are generaliy taken within fifteen minutes of the fiim deposition, and always within thirty minutes.

The portion of the film Deing studied is then completely covered with an evaporated layer of the selected compound. This produces a change, often dramatic, in the critical current, which is monitored during the evaporation. The new $I_{C}$ versus $T$ curve is then measured. Over the small temperature range usually studied, the new curve is identical in shape to that of the clean film, but is displaced along the temperature axis. This displacement is assumed to equal $\Delta T_{C}$, the change in the superconducting transition temperature produced by the compound. Botin the sign and the magnitude of $\Delta T_{C}$ are insensitive to the precise definition definition of $I_{C}$ (see Figure 9). In experiments no. 3 and 11 , Table $I$, the validity of equating curve displacement with $\Delta \mathrm{T}_{\mathrm{C}}$ was tested by actually measuring $\mathrm{T}_{C}$ both before and afier the chemical deposition. The resulting curves for no. 1 (see Figure 10), show that the lateral displacemert of the two curves varies hetween +0.06 and $+0.08^{\circ} \mathrm{K}$ as the critical current changes by more than three orders of magnitude. This displacement agrees with the measured change in $\mathrm{T}_{c}$ of $+0.076^{\circ} \mathrm{K}$. The differential nature of 

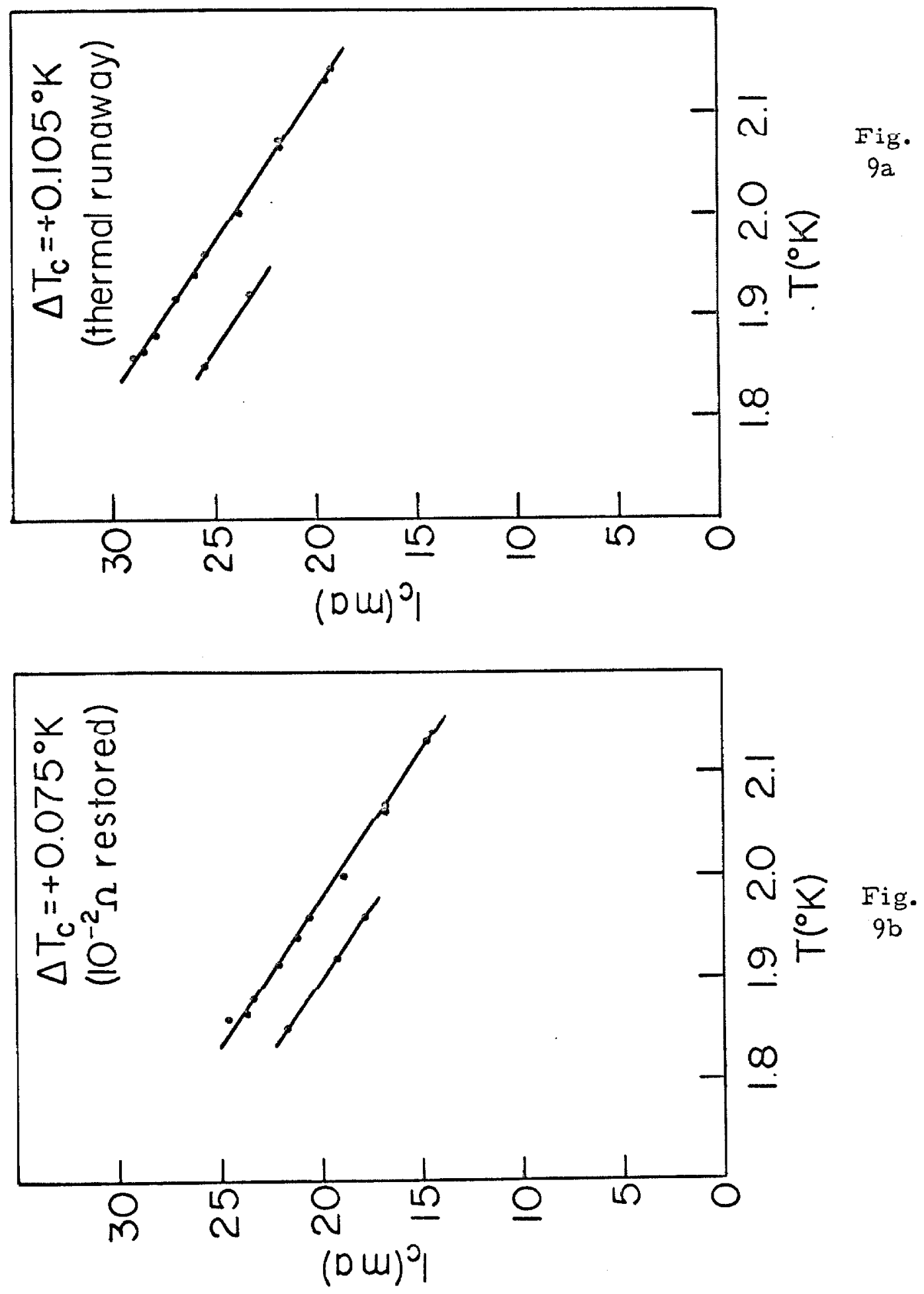

Fig. 9. $\Delta T_{C}$ ac detorminod at the point of thermal runaway and at 3 values of restored resistance. Lower curves are before, upper curves after, the deposition of tetracene. 

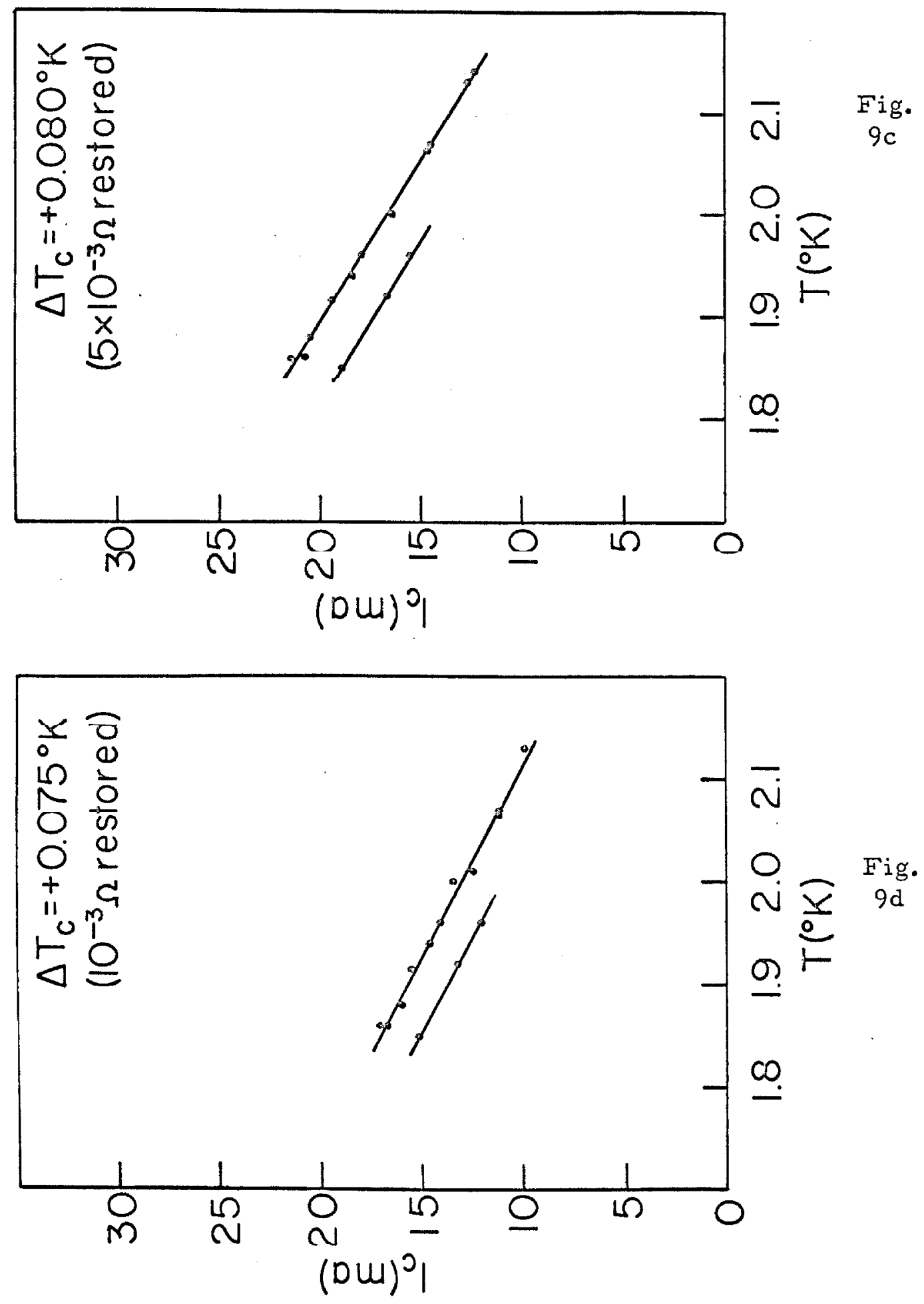


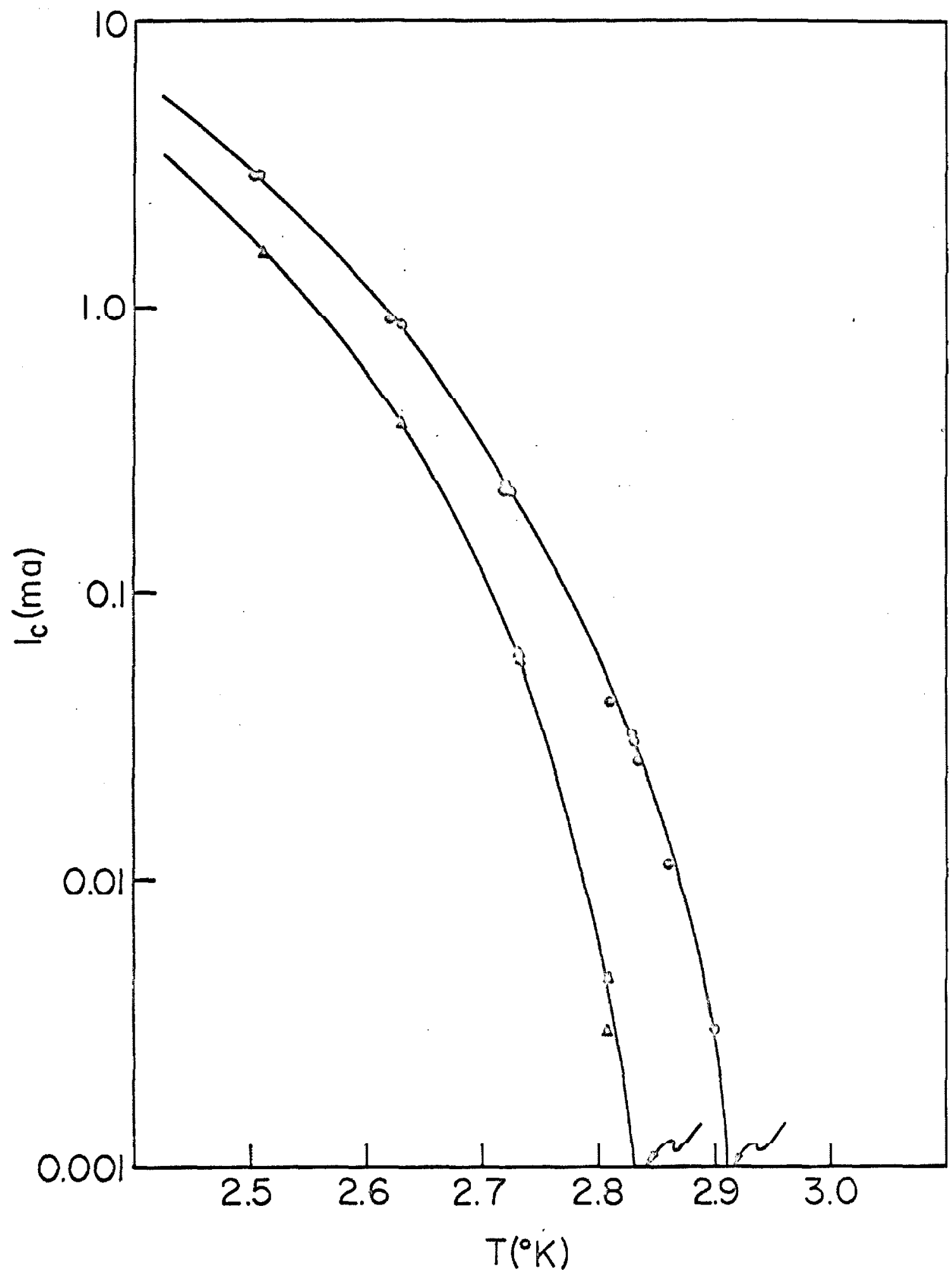

Fig. 10. Data from expt. 3, Table I. Arrows indicate measured values of $T_{c}$. Lower curve is before, upper curve is after, deposition of perylene. 
the experiment allows changes in $T_{C}$ as small as $0.025^{\circ} \mathrm{K}$ to be measured easily; it also allows a reliable determination of a zero effect. 
Experimental Results

\section{A. Film Properties}

Thin vanadium films deposited at liquid helium temperatures are quench-condensed in a high resistivity, presumably highly disordered on even amorphous form which exhibits a $T_{C}$ considerably helow $5.3^{\circ} \mathrm{K}$, that of hulk vanadium (67). These films can be annealed. After the liquid helium is exhaustea, the temperature rises slowly. Annealing begins at approximately $35^{\circ} \mathrm{K}$, and the . resistance decreases until $75^{\circ} \mathrm{K}$. After $\sim 75^{\circ} \mathrm{K}$, films show a positive temperature coefficient of resistance. The films can also be annealed to varying degrees of resistance neating with helium still present. Upon annealing, transition temperatures rise; normal film resistances drop by factors of as much as 15 to 20. We have also found that it is possible to prepare varlaciium films in a low resistivity form. This can be done by first depositing a thin Iayer of organic compound on the helium-temperature substrate, and then depooiting the vanadium film. Ono thick film ( $\sim 25 c \AA$ ) deposited directly onto the quartz was also largely annealed in formation.

Our film thicknesses were usually estimated from the room temperature resistance, $R\left(300^{\circ} \mathrm{K}\right)$, using a bulk resistivity, $P_{B}=25 \times 10^{-6}$ ohm-cm (68). The use of $P_{B}$ is consistent with the data for Iittle's films (69), which have an average roomtemperature resistivity of $21.2 \times 10^{-6} \mathrm{ohm}-\mathrm{cm}$. In addition, some high-resistivity films could apparently be nearly fully 
annealed at $4^{\circ} \mathrm{K}$ by resistance heating. This is indicated by a large decrease from the unannealed low-temperature resistance, $R(U)$, to the annealed, residual resistance, $R(A)$, and by tine large resistance ratio $\frac{R\left(300^{\circ} \mathrm{K}\right)}{R(A)}$ (particularly Table $I$, films no. 8 and 12, but also no. 6). In these cases the thicknesses determined using the differences $R\left(300^{\circ} \mathrm{K}\right)-R(A)$ and the tempenature dependent resistivity $\rho=18.5 \times 10^{-6} \mathrm{ohm}-\mathrm{cm}$ of van Beelen et al. (70) are in cxccllent agrocmont with thoso dotormincd from $R\left(300^{\circ} \mathrm{K}\right)$ and $\rho_{B}$.

For the vanadium films we have studied, a linear relationship between $R(U)$ and $R\left(300^{\circ} K\right)$ can he formintated

$$
R\left(300^{\circ} \mathrm{K}\right)=0.196 R(U)+0.153 \mathrm{~K} \text {-ohms. }
$$

This line is plotted on Figure 1l, along with the experimental data. The constants are obtained by the method of least squares, and the standard erron of estimate is $0.053 \mathrm{~K}$-ohms. This formula is used with measured values of $R(U)$ to approximate $R\left(300^{\circ} \mathrm{K}\right)$, and from this the thickness for those films for which $R\left(300^{\circ} \mathrm{K}\right)$ could not be measured.

For unannealed films of the order of $100 \mathrm{~A}^{\circ}$ thick, transition temperatures are generaliy jetween $2.2^{\circ}$ and $2.6^{\circ} \mathrm{K}$. There is a nearly linear increase in $T_{c}$ with increasing film thickness fon unannealed films as shown in Figure 12. The transition temperatures of annealed films also increased with thickness; $T_{C}$ for some of the thicker annealed films was greater than $4.2^{\circ} \mathrm{K}$. Little (69) has observed transition temperatures of 3.95 to $4.68^{\circ} \mathrm{K}$ for annealed vanadium films 170 to $100 \mathrm{C} \mathrm{A}^{\mathrm{O}}$ thick, the 
82

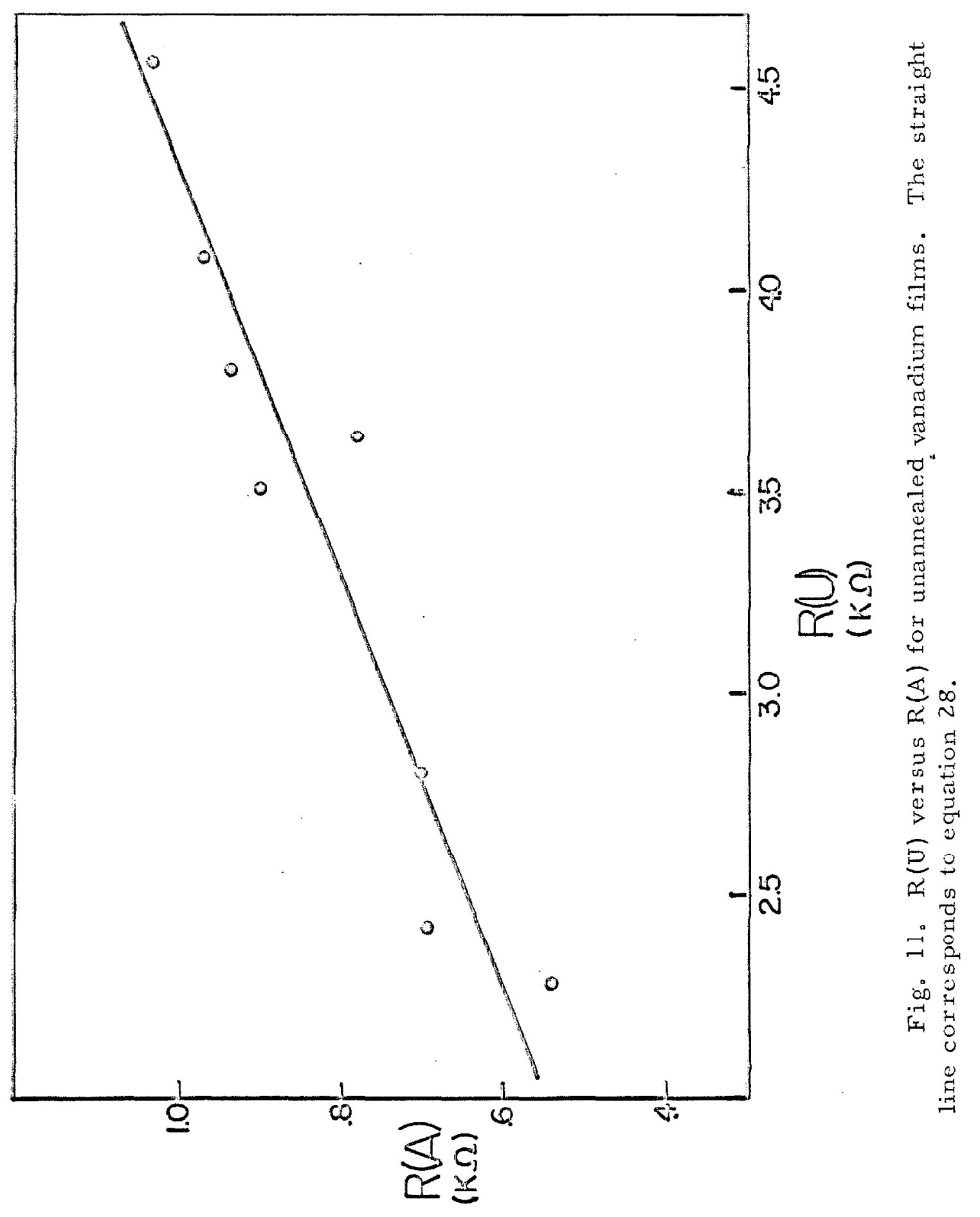




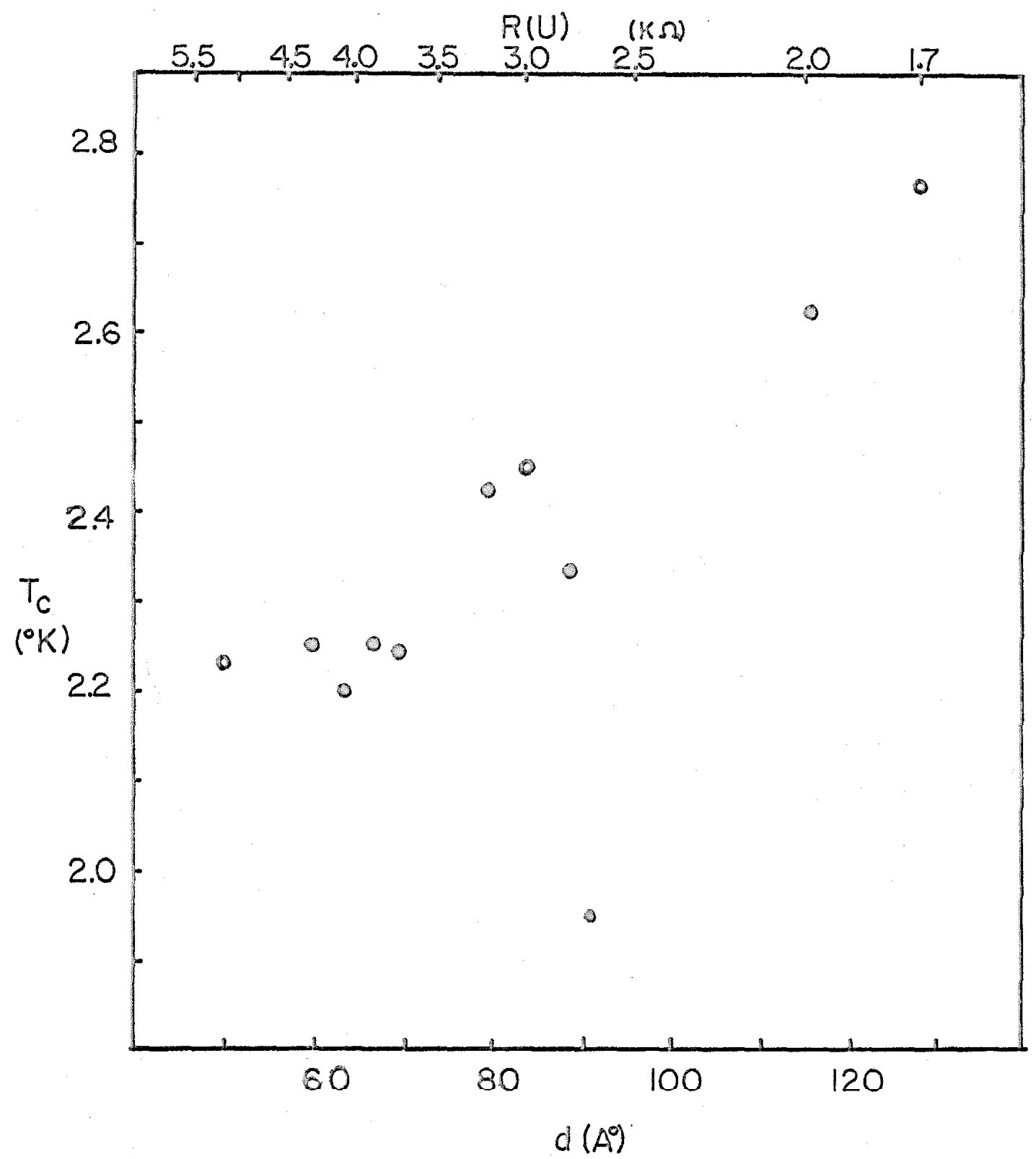

Fig. 12. $T_{C}$ versus thickness (d) for unannealed vanadium films. The $R(U)$ scale is determined from d using equation 28 and the room temperature resistivity.

- d calculated from R(A).

- d calculated from $R(U)$ and equation 28. 
thicker films naving generajly higher $T_{C}$. Van Beelen et ä. (70) have seen transition temperatures from $I .8$ to $4.6^{\circ} \mathrm{K}$ for thicknesses between 130 and $800 \mathrm{~A}^{\circ}$.

Indium films quench-condensed on a $4^{\circ} \mathrm{K}$ quantz surface were superconducting above the bulk transition temperature $\left(3.4^{\circ} \mathrm{K}\right)$, as expected (71). The normal resistance of these films was recuced by a factor less than four on annealing.

Measurements of de critical currcnto have bocn adoquato to establish the existence of the effects we were seeking. We have been able to study "isothermal" transitions (Fig. 8) and the results are independent of restored resistance (Fig. 9). There have been several attempts to determine critical currents for thin films from theory. Ginzburg (72) found that

$$
I_{c}(T) \propto\left(T_{c}-T\right)^{3 / 2}
$$

for $T$ very close to $T_{C}$. Since this relationship has been verified in other investigations (73) it is worthwhile to test our critical current measurerents against it. In particular, if joule heating is important, critical curments should increase less rapidly than predicted by equation 29, since the heating will tend to drive the film normal before the "true" critical currenl. Figure 13 is a $\log -\log$ plot of $I_{C}(T)$ versus $\left(T_{C}-T\right)$ for the two experiments on which such a test can be performed. The curve for experiment 11 has a slope of 1.8 , slightly greater than the value predicted by equation 29. That for experiment 3 approaches $3 / 2$ as $\left(T_{c}-T\right) \rightarrow 0$, but at larger values the slope 


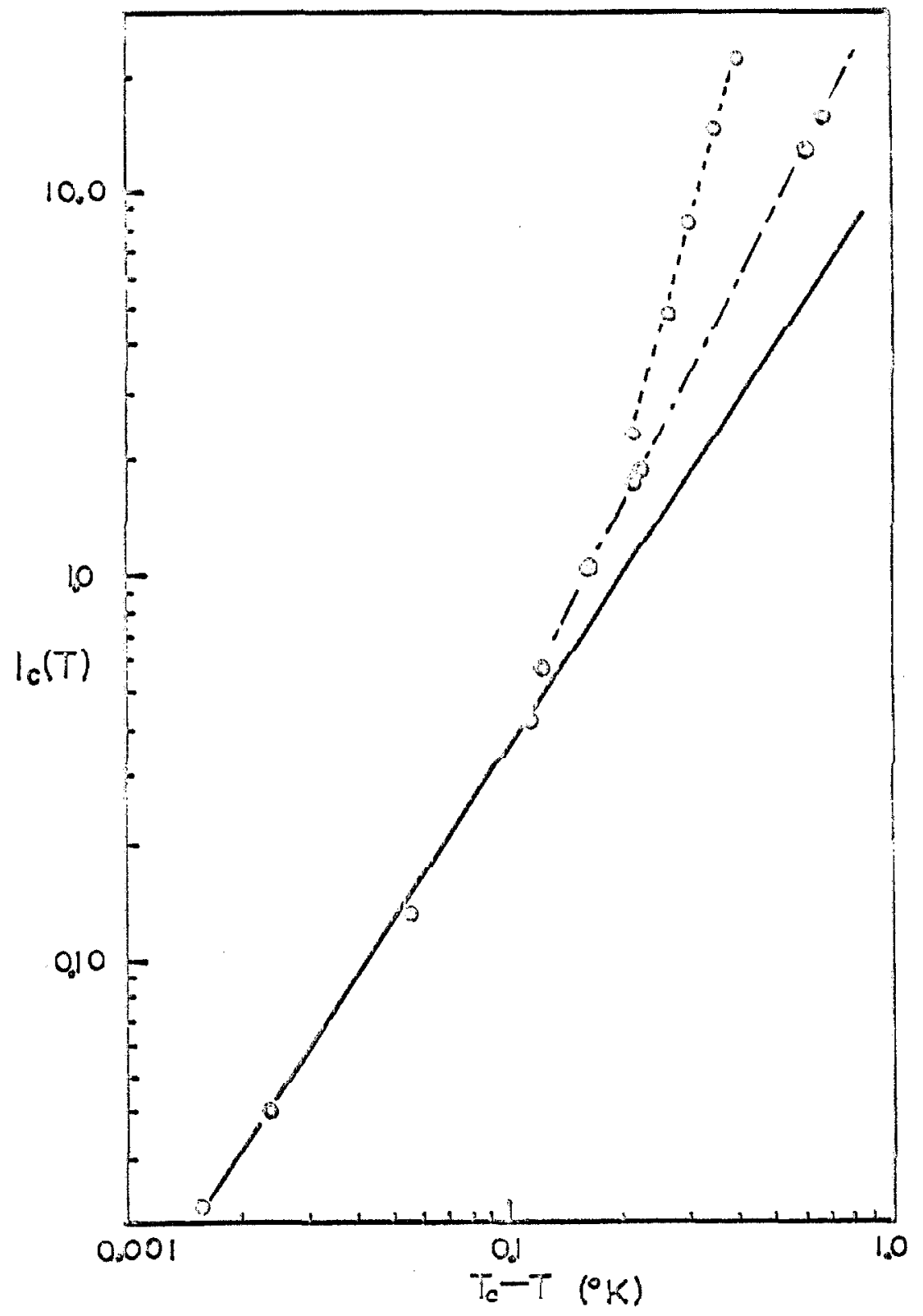

Fig. 13. Log-log plot of temperature variation of critical current. Currents are normalized so that lowest points fall on solid line of slope $3 / 2$.

- Expt. 3, Table I. Oxpt. I1, Table I.

$\left.\begin{array}{ll}\text { Straight line of slope } 7 / 2 \text { for expt. I } \\ \text { Straight line of slope } 1.87 \text { for expt. II }\end{array}\right\} \begin{gathered}\text { arge values } \\ \text { of } \mathrm{T}_{\mathrm{c}}-\mathrm{T}\end{gathered}$ 
increases to $7 / 2$, insteac of decreasing as might be expected if heating were becoming important. Whether these unexpectedly large slopes result from the fact that our films are much thinner than those used in previous comparisons to equation 29, or from some peculiarity of thin vanadium tilms can only be aecided by furtiner experiments. In any event, it is gratifying that the slopes approach to the expected value in the region where the theory is most applicable, $\left(\mathrm{T}_{C}-\mathrm{T}\right) \cdots 0$, and that aeviations arc not in the direction indicative of joule-heating difficulties.

It must be pointed out, howeven, that $I_{c}(T)$ versus $T$ curves generally show discontinuities at the $\lambda$-point. These nave also been obsenved by others (74), and are due to the large increase in the thermal conductivity of liquid helium as it becomes superfluid. Their existence indicates that heating of some kind still plays a role in determining $I_{C}$. We believe that this heating is the result of that small amount of room temperature radiation reaching the sample, rather than of the impressed currents.

\section{B. Chemical Efiects}

Experimentai results for the organic chemicals studied are cisplayed in Table $I$. It can be seen that the values of $\Delta \mathrm{T}_{\mathrm{c}}$ for vanadium films fall into two groups which are approximately symmetric about $\Delta \mathrm{T}_{\mathbf{c}}=0$; most enhancens increase $\mathrm{T}_{\mathrm{c}}$ by $0.06^{\circ}$ to $0.11^{\circ} \mathrm{K}$ and depressors decrease $\mathrm{T}_{\mathrm{C}}$ by $\sim 0.05^{\circ}$ to $0.10^{\circ} \mathrm{K}$, for films with $T_{C}$ generally between 2.2 and $2.6^{\circ} \mathrm{K}$. A correlation of the sign of $\Delta r_{C}$ with the properties of the molecules studied is discussed in section IV. The two experiments with indium films 


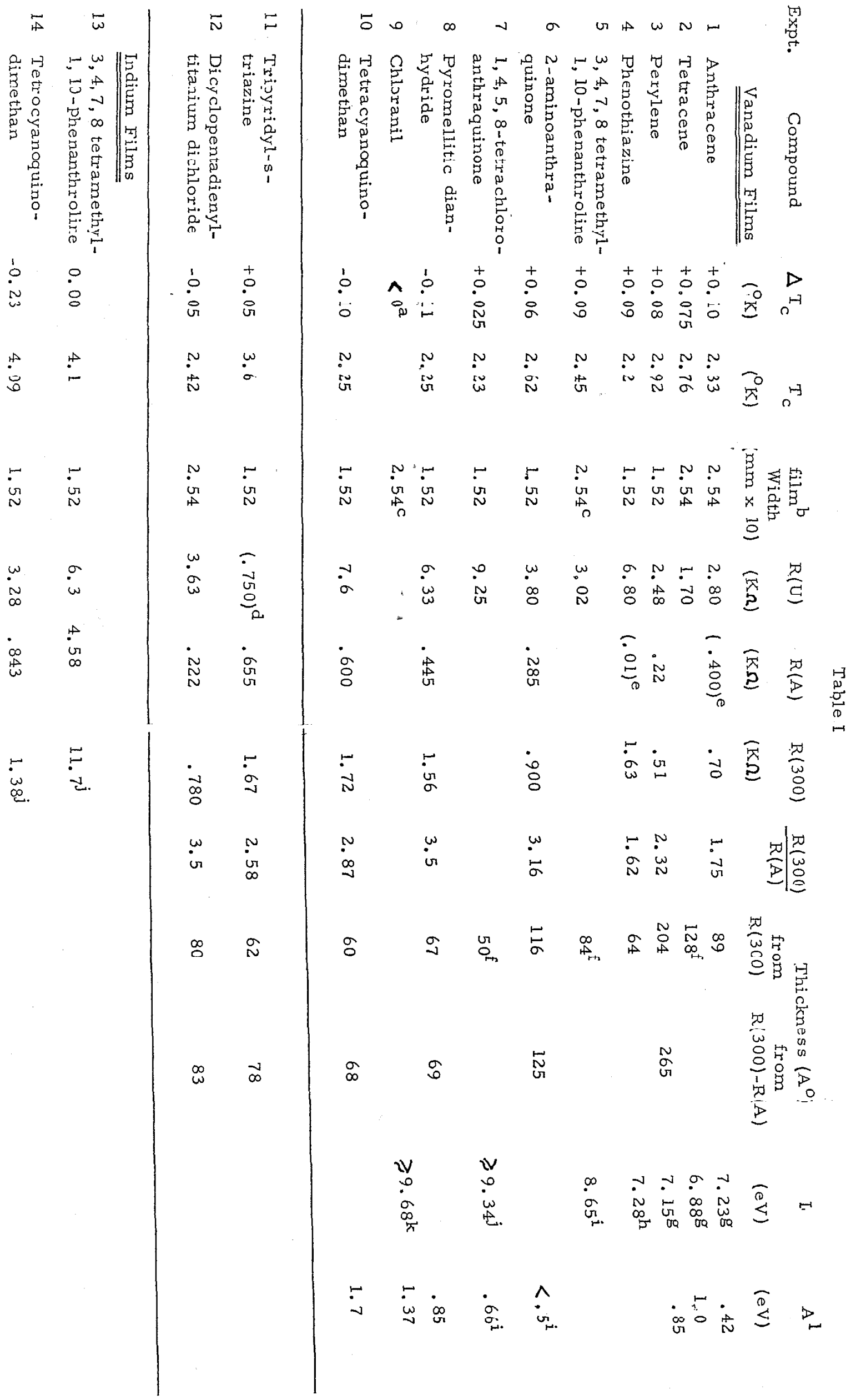




\section{Legend to Table I}

(a) Film was ciestroyed after the initial critical current depression was observed, but before $\Delta \mathrm{T}_{\mathrm{c}}$ could be measured.

(b) All films, $6.35 \mathrm{~mm}$. long.

(c) Newhouse geometry not used.

(d) Vanadium was deposited in an essentialiy annealed form. Experiments with this compound on unannealed films were similar.

(e) Not fully arnealed.

(f) Estimated from $R(U)$ as cescribed in text.

(g) Trom "Introduction to Naso Spectromctry" by Robcrt W. Kiccr (Prentice-Hall, Englewood Cliffs, N. J., 1966), Appendix IV.

(h) From M. Kinoshita, Bul]. Chem. Soc. Jap., 35, 1609, 1962.

(i) Estimated in this laboratory from charge-transfer absorption spectra.

(j) Ionization potential of unsubstituted anthraquinone $=9.34$.

(k) Ionization potential of unsubstituted quirione $=9.68$.

(1) From G. Briegleb, Angew. Chem. Internat. Edit., 3, 617 (1964), except where noted. 
were primarily performed as tests of suggestions which were advanced about the mechanism producing changes in $\mathrm{T}_{\mathrm{C}}$, and are also discussed.

Experiments fon two organic compounds, metal-free pthalocyanine $\left(\Delta \mathrm{T}_{\mathrm{C}} \sim-0.05^{\circ} \mathrm{K}\right)$ and 5-nitro-1, $1 \mathrm{U}$-phenanthroline $\left(\Delta \mathrm{T}_{\mathrm{C}} \sim\right.$ $+0.05^{\circ} \mathrm{K}$ ) are not included in the table because poor vacuums dur: ing those experiments made it likely that the chemicals were deposited onto highly contaminated surfaces, makirly the resuzls. suspect.

Table II contains the data for the two inorganic compounds, oxygen anci thallium chloride, whose effects have bcon otudicd.

Table III presents the data for four experiments with 3, 4, 7, 8-tetramethyl-1, 10-phenanthroline (IMP) deposited on vanadium filme, including experiment 5 of Table I. Since experiments 1-3 were done with rather poor vacuum conditions, a superfluic helium leak required that experiment 3 be carried out above the $\lambda$-point, and none of these expeniments employec the Newhouse geometry, we consider this spread in values to give an upper Iimit to our experimental error. With the improved experimental conditions that have obtained since these, our very first experiments, much better fiIms, with more nearly uncontaminated surfaces, can now be studied. Expeniments with such films should allow better reproducibility for a given compound, and quantitative comparisons between results for different compounds. In the present results, the variation in film properties and experimentai conditions between expeniments makes the variations in magnitude 
m

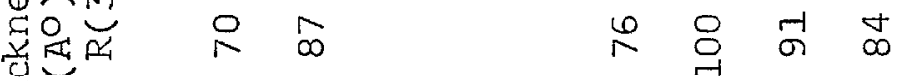

㤩

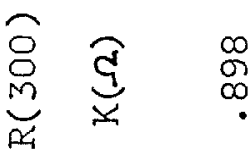

o

离 乌

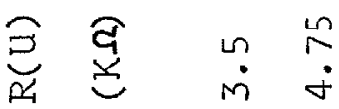

$\begin{array}{lll}\leftarrow & \stackrel{y}{0} & 0 \\ \dot{0} & \dot{v} & \dot{m}\end{array}$

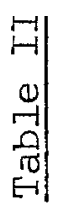

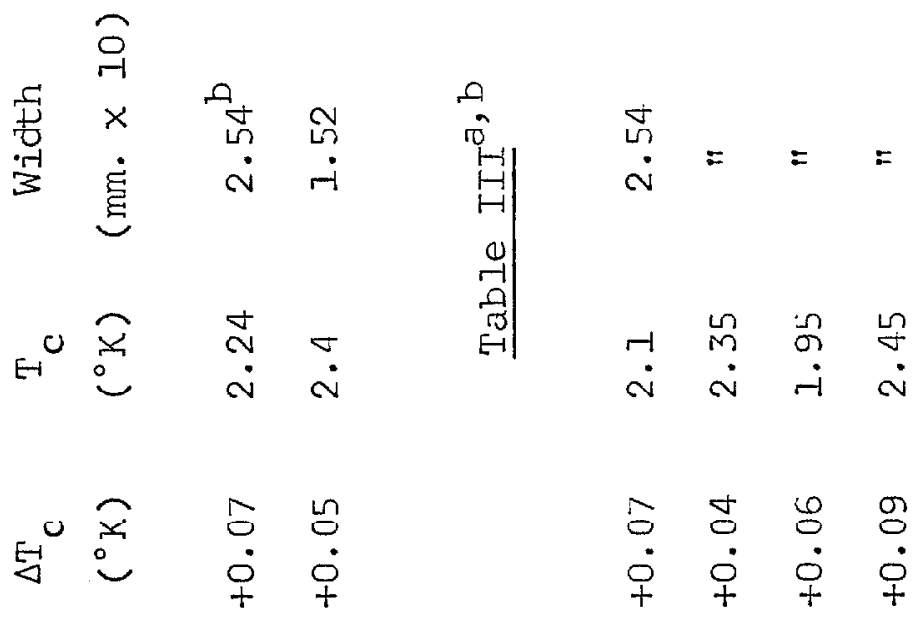

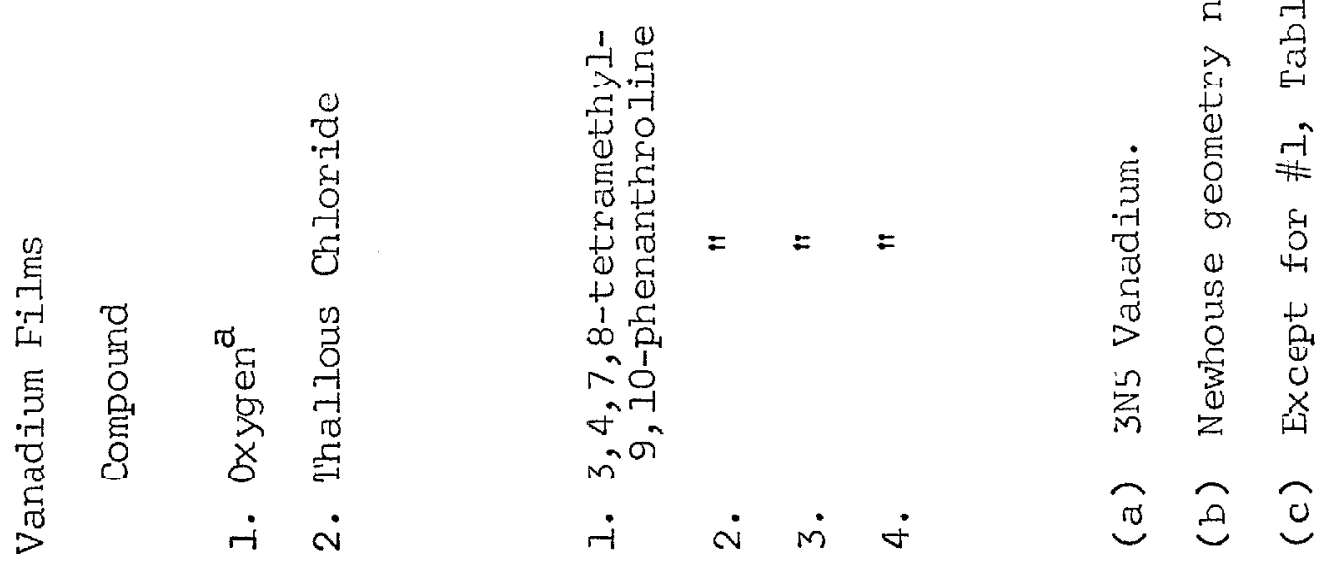


of $\Delta T_{C}$ within the enhancen and depresson groups of perhaps secondary significance.

There is an experimental observation which strongly supports the suggestion that there is a strong chemical interaction between these molecules and a metal surface, even at liquid helium temperatures. Deposition of the colorless compound, TMP, onto a varadium film held at $4.2^{\circ} \mathrm{K}$ produced a reddish-purple color, indicative of a chemical reaction.

It should be mentioned that the occurnence of both positive and negative values of $\Delta T_{C}$ rules out most aiternatives to the interpretation that the observed effects are chemical in nature. Since the physical properties of all the organic chemicals are so similar, any suggestion involving a physical change in tilm properties caused by the mere deposition of a chemical would predict on $y$ y one sign for $\Delta_{c}^{m}$.

In addition, the fact that hydrogen is usually the primary gas released during the evaporation of both erhancers and depressors eliminates the possibility that it is the evolved gases, rather than the chosen chemical, that cause the observed effects. 


\section{DISCUSSION}

The experiments described in the preceding section have clearly demonstrated that it is possible to either raise or lower the critical temperature of thin metal films by the deposilion of an appropriate chemical on the film surface; as suggested in part II, Interaction Mechanisms, the intimate association of molecules with a metal doce influonco the superconducting properties of the metal. What is more, the sign of $\Delta \mathrm{T}_{C}$ for the aromatic compounds studied can be related to a simpie division of the molecules by electronic properties. Molecules with low electronegativity, $\psi$, are enhancers, those with hign electronegativity are depressors. We use Mulliken's definition (75), $\psi=1 / 2(I+A)$, where $I$ is the finst ionization potential and $A$ the electron affinity. As can be seen in Table I, for many of these compounds I and $A$ are not both known. However, if the compounds are divided into enhancers and depressons, it is seen that among the planar aromatic molecules all depressors act as acceptors in intermolecuiar charge-transfer complexes, while most of the enhancers are either well-known charge trans fer donors (anthracene, tetracene, perylene, phenothiazine) (76) or can function as such (TMP) (77). Since acceptors characteristically have high $\psi$ while donors have low $\psi$, the division of these eninancers and depressors on the basis of electronegativity is immediate.

Also among the enhancers are the substituted arthraquinones. These were chosen because it was expected that as $\psi$ was 
systematically decreased, the sign of $\Delta \mathrm{T}_{\mathrm{C}}$ would change at some point. They are weak charge transfer acceptors whose electmon affinity values are less than those of tetracyanoquinodimethan (TCNQ), chloranil, and pyromellitic dianhydride (PMA) (78). If we consider the electronegativities for the charge-transfer acceptors to paralle their own A values, then all tine planar aromatic compounds studied can be arranged according to $\psi$ and a division made which separates enhancens from depressons, this divisior. coming between PMA and I, 4,5,8-tetrach loroanthraquinone. The two adaitional organic chemicals studied, tripyridyls-triazine (TPTZ) and dicyclopentadienyl-titaniumichlonide (DCT) are not planar aromatics. They can, nowever, be reasonably arranged by electronegativity with the planar compouncis, and the signs of $\Delta \mathrm{T}_{c}$ correspond to the above division. The pyridine rings of TPTZ $\left(\Delta \mathrm{T}_{\mathrm{C}}=+0.05\right)$ are sterically prevented from all lying in the plane of the triazine ring, but the molecule should still be a weak electron donor with between that of TMP and the anthraquinones; thus it should be possibie to place it by electronegativity with the other enhancers. $D C T\left(\Delta T_{C}=-0.05^{\circ} \mathrm{K}\right)$ is readily reduced, indicating the ease of the reaction. $\mathrm{Ti}(\mathrm{IV})+\mathrm{e}^{(-)} \rightarrow \mathrm{Ti}(\mathrm{III})$ and the cornespondingly high electronegativity of the molcculc; it can thorcforc ncasonably be placed with the other depressors.

Despite our previously expressed doubts as to the significance of the relative magnitudes of $\Delta \mathrm{T}_{\mathrm{C}}$, there is discennible a systematic variation of $\Delta \mathrm{T}_{\mathrm{C}}$ within the enhancers that should at 
least be noted. Experiments $1-10$ in Table $I$ are arranged in order of increasing $\psi$ for the organic chemical. Among the enhancers, there does seem to be a decrease of $\Delta T$ with increasing $\psi$ (and I), particularly in experiments 4-7.

That such a simple correlation can serve to classify all the organic chemicals studied as enhancers and depressors should, perhaps, be surprising. As an indication of this, we might consider the first enhancer to be discovered, TMP. Vanadium atoms. can occur in the zero valence state in $V^{\circ}(T M P)_{3}$ complexes (40). It was therefore suggested that any observed effects with TMP might be attributable to chelation of sumfane atoms. Although a $V(T M P)_{3}$ complex would clearly be impossible, one could conceive of a surface vanadium complex $V(V)_{X}$ (TMP) where the remaining "ligands" are the neighbors of the surface vanadium atom. TPTZ, the second enhancer found, and also a potential chelating agent, might act similarly.

It is clear, however, that the aromatic hydrocarbons cannot be considered to act by chelation. It was only when we considered TMP, not as sitting perpendicular to a film surface, "biting" into a vanadium atom, but as lying flat on it, that the electron donor-acceptor behavior of these molecules and therefore their values, could be used to correlate their behavior.

It should not be surprising that, once the molecules are considered on the basis of electronegativity, the division between enhancers and depressors does not come at an obvious point in terms of intermolecular charge-transfer: 
3,4,7,8-tetrachloroanthracuinone, an accepton in charge-transfer complexes, is yet an enhancer. Trie qualilalive division between donors and acceptors, Iow and high. $\psi$, can only be macie with respect to some arbitrary reference. In charge-transfer complexes benzone is frequently used as an implicit reference. In this study the division by electronegativity is made with a vanadium filr. surface as the reference.

This, the proposed division really suggests that the quantity which determines the sign of $\Delta T_{C}$ is $\Delta \psi=\left(\psi_{\text {iktal }}-\psi_{\text {ilol }}\right)$. Since it follows from the definition of $\psi$ that $\psi_{M_{1}}=\phi_{M}$, the metallic work function, this can be rewnitten $\Delta \psi=\left(\not \partial-\psi_{m}\right)$. Then the criterion for $\Delta T_{C}$ is as follows:

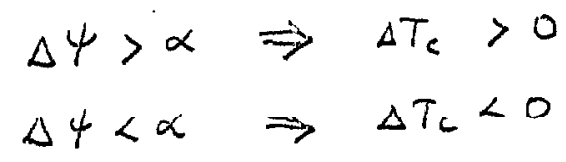

where $\alpha$ is some constant which máy be zero.

Il is possilile to melate $\Delta \psi$ to the propertico of a molecule-metal surface bond by assuming a simple, threeconfiguration wave function to describe the bond (75);

$$
\begin{aligned}
|W\rangle & =a\left|M^{0} x^{0}\right\rangle+b\left|M^{*} x^{-}\right\rangle+c\left|M^{-} x^{+}\right\rangle \\
& =a|a\rangle+b|b\rangle+c|c\rangle
\end{aligned}
$$

The bond polarity is detemined by $/\left.b\right|^{2}-|c|^{2}$, and the sign of inis quantity is largely determined $\nu y \Delta \psi$. 
In an obvious notation the secular equations for the coetificients and energy are:

$$
\begin{aligned}
& a\left(E_{a}-E\right)+b\left(H_{a b}-S_{a b} E\right)+c\left(H_{a c}-S_{a c} E\right)=0 \\
& a\left(H_{b a}-S_{b a} E\right)+b\left(E_{b}-E\right)=0 \\
& a\left(H_{c a}-S_{c a} E\right)+c\left(E_{c}-E\right)=0
\end{aligned}
$$

The off-diagonal elements involving two-electron transfer are assumec to be zero. The diaconal matrix elements are

$$
\begin{aligned}
& E_{a}=0 \\
& E_{b}=\phi-A_{x}-\varepsilon \\
& E_{c}=I_{x}-\phi-\varepsilon^{\prime}
\end{aligned}
$$

where I, $A$, dul $\phi$ llave been defined, and $\delta$ and $\varepsilon^{\prime}$ are stabilization energies for the negative and positive ions, respectively, at the metal surface.

The ground state solutions for $b$ and $c$ (enengy $E_{0}$ ) are

$$
b=-a \frac{\left(H_{b a}-S_{b a E}\right)}{E_{b}-E_{0}} \quad c=-a \frac{\left(H_{c a}-S_{c a} E_{0}\right)}{E_{c}-E_{0}}
$$

If the simplifying assumption is made that the numerators are equal

$$
H_{b a}-S_{b a} E_{0}=H_{c a}-S_{c a} E_{0}
$$


then $b$ and $c$ have the same sign anc

$$
|b| \geqslant|a| \text { if }\left(\frac{1}{\varepsilon_{0}-E_{0}} \geqslant \frac{1}{E_{0}-E_{0}}\right)
$$

which leads to

$$
|b| \geqslant k \mid \text { if } \Delta \psi \geqslant\left(\frac{\varepsilon-\varepsilon^{\prime}}{2}\right)
$$

The direction oif this inequality, then determines the bond polarity. It is of course true that the magnitudes of $a$ and $b$ are determined by $I, A$, and $\phi$ individually, and that $\Delta \psi$ only determines winich coefficicnt io largor.

The division by electronegativity coes rot, however, specify the detailed mechanism by which the changes in superconducting properties are produced. In particular, the obvious suggestion is that the effects in some way follow from simpie electrostatic charging: thus, the molecule would donate or accept an electron from the metal and become a positive on negative ion at the surEace. Fortunately, things seen to be more complicated, and therefore more interesting, than this.

Static charging experiments have been performec on indium anc tin films by Gloven and Sherrill (GS) (78), and by Stadier (79). GS prepared parallel plate capacitors with a superconducting thin film as one plate. By charging the capacitor, with the experimental film positive (or negative) electrons could be withdrawr. (on acided) and the effect on $T_{C}$ could be observed. They found that adding electrons to an indium film lowers $T_{c}$, and that 
subtracting electrons raises it. That is, if $\mathrm{n}$ is the electron concentration, $\mathrm{d}_{\mathrm{r}} \mathrm{c} / \mathrm{dn}<\mathrm{U}$. However, we observed that deposicing TCNQ, a very strong electron acceptor, on an indium film gave $\Delta T_{C}=-0.23^{\circ} \mathrm{K}$ (Tabie I, expt. 14), the wrong sign for simple electron withdrawal. This result seems to unambiguously elininate static charge transfen as the sole means by which TCNQ affects $T_{C}$ of an indium fiIm.

Finthen, although mone indiment, evidenne against the static charging hypothesis comes from considering Table II, expt. 2, in which oxygen deposited on the surface of a vanadium fiim increased $T_{c}$ by $0.07^{\circ} \mathrm{K}$. Rith $(80)$ has studied the effects of oxygen on the transition temperatures of films of metal from groups IIIa ard IVa. Where his nesults could be compared with those of GS (In and $S_{n}$ ) they were completeiy interpretable on the basis of charge transfer. Electrons are withorawn from the films by the activaled reduclion of rolechiar uxygen on the diedal surface. Act:vation temperatures for the several metals ranged from $3^{\circ} \mathrm{K}$ to $25^{\circ} \mathrm{K}$.

The reduction of molecular oxygen on transition metal surfaces is usually Delieved to be non-activated (81). Thus the increase of $T_{c}$ of vanadium by oxygen is reasonably interpreted as a result of the withdrawal of electrons by the reduction of oxygen in a process with an activation temperature of less than about $2^{\circ} \mathrm{K}$. This interpretation asserts that actual electron witharawal from a vanadium film, as with an indium film, increases $\mathrm{T}_{C}$. Since aromatic molecules of high electronegativity 
decrease the $\mathrm{T}_{\mathrm{C}}$ of vandium and indium, this lends further support to the thesis that organic molecules do not change $T_{c}$ by simple static charge transfer.

The results of the static charging experiments on the various metals can be understood simpiy. In section II, Interaction Mechanisms, the BCS result was given, $T_{C}=1.14 \theta_{D} \exp$ $\left(-\frac{I}{N\left(E_{F}\right) V}\right)$. Trerefore, if electron adoition or withcrawal charges $E_{k}$ slight $I_{y}$, it will also in general crange $N\left(E_{F^{\prime}}\right)$ and therefore $T_{C}$. The different sign of $\mathrm{dT}_{\mathrm{C}} / \mathrm{dn}$ for different metals can be understood by considering the simple density of states curves below:

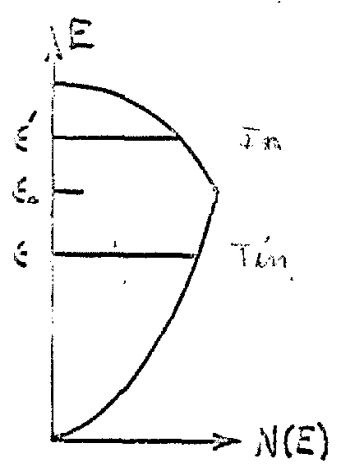

For an n-type netal, such as tin, with $E_{F}=\epsilon<\varepsilon_{0}, \frac{\mathrm{dN}(E)}{\alpha E} / E_{F}>0$. Adding electrons $w i=$ increase $E_{F}, N\left(E_{F}\right)$, and therefore $T_{C}(\dot{a T} / d n>0)$. Fon a p-type metal, such as indium $E_{F}=\epsilon !>\epsilon_{0}$, $\frac{d N(E)}{d E} / E_{F}<0$, and therefore $\mathrm{dT} / \mathrm{dn}<0$.

Now low-temperature specific heat measurements on 3-d transition metals and their alloys have given a cunve for the electronic specific heat coefficient versus the number of valence electrons in the alloy (34). If the simple one-electron picture is adopted and a rigid band assumed, a conversion of the electron concentration scale to an energy scale leads to the corresponding 
density of states curve (84). It is found that $\mathrm{dN}(\mathrm{E}) / \mathrm{dE}<0$ at the fermi level of vanadium. The above explanation of static charging effects would then predict that electron witndrawal should increase $\mathrm{T}_{C}$ for vanadium, just as with indium. This is, again, consistent with the results for oxygen, but in opposition to those with organic donors and acceptons.

up to this point, a correlation between moleculan properties ard the effects on superconductivity has been made, and argumento have beer. presented to indicate at least one microscopic mechanism that this correlation does not imply. There is one pair of. experiments which may point in the dinection of such a merhanism. TMP deposited on a vanadium film increased $\tilde{\Sigma}_{C}$ (expt. 5, Table $I$ ), while deposited on an indium Eilm it gave no effect (expt. 13, Table I). The bonds of molecuies to transition metal surfaces are generally consiciered to be largely covalent and dependent on partially filled d-orbitals, as in the metallocene compiexes of transition metals and aromat:ic molecules (40). The null effect of TMP on the non-transition metal indium, which has no unfilled d levels, may indicate that the enhancements seen on vanadium are relatec to $d-\pi$ bonding between unfillec $d$-levels of the metal and the molecular n-orbitals.

Several medianlisms have Deen suggesied in sechion II, Interaction Mechanisms, as being capable of enhancing superconductivity in the presence of strong metal-molecule bonds. As to which--if any--of these are important, there is really little more that can be unambiguous ly drawn from this study. 
It is still possible to speculate on the reason for the depressions. A simple suggestion 1s that the strong electror acceptors abstract an electron from the metal, forming radical anions on the surface. Magnetic interactions between the radical and the conduction electrons could overbalance the tendency of e-ectron-withdrawal to increase $T_{C}$ of these netals, causing a net reduction in $T_{C^{*}}$

However, there are other suggestions which would involve strong, non-magnetic, coupling between molecule and metal. One was anticipated in the discussion of conductive conjugation, where it was suggested that interaction with a molecule might possibly lead to an increase in the density of states away from the Fermi surface of the metal, Dut a decrease at the Fermi surface. Both depressors and enhancers have ionization potentials greater than the work function of vanadiun, and therefore would have their filled molecular orbitals located lower in energy than the Fermi level. Since the depnessons have greater ionization potertials than do the enihancers, their filled molecular orbitals will lie farthen below the Fermi surface than do those of the eninancers; they would thus be more likely to cause this unfavorable change in the density of states.

I second speculation is that the electrons in the molecules with high electronegativity may be iess mobile, and therefore less capabie of providing screening of electron-electron colilomb interactions. Thus, if the molecular orbitals of such a molecule were mixed with metallic conduction states, the interaction 
would be less favorable, and possibly even repulsive, in the region of the molecule.

Two interesting observations may be made after the preceding remarks about $\psi$, screening, and $\Delta T_{c}$. First, most of the enhancers are bigger molecules--more benzene rings--than are the depressors. This might be correlated with increasing shielding in the presence of bigger T-electron systems. The second observaLiun is Jased un anuliten', perilaps unwartamiled, comparison of values of $\Delta T_{C}$ for different compounds. The compounds in experiments 1,6 , and 7, Table I, all have the three-ringed anthracene structure: anthracene, 2-aminoanthraquinone, anci 1, 4, 5, 8,-tetrachloroanthraquinone. There is a definite decrease in the magnitude of $\Delta T_{C}$ as $\psi$ increases from one of these compounds to the next.

We have not succeeded in determining the microscopic nature of the effects we have observed. However, we feel that the possibility of correlating molecular properties with molecular effects on superconductivity in what is yet a preliminary investigation of a new phenomenon augurs well for an eventual understanding of the mechanisms involved, and for the profitable extension of these studies to other systems. 


\section{REFERENCES}

1. Some general references to Superconductivity are:

(a) F. Iondon, "Superfluids," Vol. I (John Wiley anci Sons, 1950).

(b) E. A. Lynton, "Superconcuctivity" (Methuen Monographs, 1962 ).

(c) M. Tinkham, in "Low Temperature Physics," ed. Dewitt, Dreyfus, and de Gennes (Gordon and Breach, 1962).

(d) J. Bardeen and J. R. Schrieffer, Vol. III, Progr. in Low Iemp. Pnysics.

(e) P. G. de Gennes, "Superconductivity," (W. A. Benjamin, 1966).

2. H. Kammerlingh Onnes, Comn. Phys. Lab. Univ. Leiden, II9, 120,122 (1911).

3. F. Block, Z. Physik., 52, 555 (1928); 53, 216 (1929); 59, $208(1930)$.

4. W. Neissner and R. Ochsenfeld, Naturwiss., 21, 787 (1933).

5. C. J. Gorter anc H. B. G. Casimir, Pnysica, I, 306 (1934).

6. F. London, Phys. Rev., 74, 562 (1948) and Ref. la, Chap. E.

7. Ref. I(a), Chap. E.

8. Estimates from J. R. Schrieffer, "Superconductivity" (W. A. Benjamin, New York, 1964).

9. H. Fröhlich, Phys. Rev., 79, 845 (195C).

10. E. Maxwell, Phys. Rev., 78, 477 (1950).

11. Reynolds, Serin, Wright and Nesbitt, Phys. Rev., 78, 487 (1950).

12. Ref. Il and J. Bardeen, Phys. Rev., 8C, 567 (1950).

13. N. R. Schafroth, Helv. Phys. Acta, 24, 645 (195I).

14. A. B. Migdal, Soviet Phys. JETP, $\frac{1}{m}, 996$ (1958). 
15. J. Bardeen, L. N. Cooper, and J. R. Schrieffer, Phys. Rev., 108,1175 (1957).

16. L. N. Cooper, Phys. Rev., 104, 1189 (1956).

17. N. N. Bogoliubov, D. N. Zubarev dill Yu. A. Tsemikov, Dokl. Acad. Nauk SSSR, 117, 788 (1957).

18. L. F. Gorkov, Soviet Ihys. JETP, 7, 505 (1958).

19. D. H. Douglass and L. M. Falicov, Progr. Low. Temp. Phys., IV, 97. (I96I).

20. Quoted in T. H. Geballe and B. T. Matthias, Ann. Rev. Phys. Chem., 14, 141 (1963).

21. R. A. Hein et al., Phys. Rev. Letters, I2, 230 (1964).

22. B. T. Matthias, Prog. in Iow Temp. Phys., II, 138 (1957).

23. (a) Ru: Geballe, Matthias, Hull, and Corenzwit, Phys. Rev. Letters, 6 , 275 (1961).

(b) Os: B. T. Matthias, J. Phys. Soc. Japan, 17, 104, Supplement B-I ( 1962$)$.

(c) Nb3S: G. E. Devlin and E. Corenzwit, Phys. Rev., 120, $1964(1960)$.

24. P. Morel and P. N. Anderson, Phys. Rev., 125, 1263 (1962).

25. J. W. Garland, Phys. Rev. Letters, II, III (I963); II, II4 (1963).

26. Matthias, Compton, Suhl, and Corenzwit, Phys. Rev. 115, 1597 (1959).

27. See T. H. Geballe, Rev. Mod. Phys., 36, I34 (1964).

28. L. V. Keldysh, Soviet Phys. Uspekki, 8,496 (1965).

29. B. T. Matthias in "Superconductors," ed. by M. Tannenbaum and N. V. Wright (Interscience Publishers).

30. See ref. 1,d for a review of this topic.

31. H. M. McConnell, unpublished.

32. W. A. Little, Phys. Rev., 134A, I4I6 (1964).

33. G. Chanin, E. A. Lynton, and B. Seraphim, Phys. Rev., 114, 719 (1959). 
34. Cheng, Gupta, van Reuth, and Beck, Phys. Rev., 126, 2030 (1962).

35. Screening is discussed in R. Brout and P. Carruthers, "Lectures on the Many-Electron Problem" (Interscience Publishers, 1963).

36. See C. Kittel, "Quantum Theory of Solids" (John Wiley and Sons, 2963).

37. F. Sunl, B. T. Matthias, and I. R. Walker, Phys. Rev. Letters, is, $552(1959)$.

38. J. Kondo, Prog. Theoret. Physics., 29, I (1963).

39. G. Wilkinson and F. A. Cotton, Prog. in Inorg. Chem., P. I, Vol. I ( 2959 ).

40. S. Herzog and $\mathrm{H}$. Prakel, 8th ICCC, Vienna, 1964.

11. L. Ongel, "Mn Introduction to Inansition Mhemistry" (John Wiley E Sons, 1960); see especially p. 134.

42. M. H. Cohen, Rev. Mind. Phys., 36, 243 (1964).

43. (a) V. L. Ginzburg, Physics Letters, 13, 101 (1964).

(b) B. T. Geilekrnan, Soviet Phys. UETP, 21, 796 (1965).

(c) W. L. McCubbin, Physics Letters, 19, 461 (1965).

44. (a) R. A. Ferrell, Phys. Rev. Letters, 13, 330 (1964).

(b) R. E. De Wames, G. W. Lehman, anci T. Wolfram, Phys. Rev. Letiens, 13, 749 (1964).

45. R. E. Merrifield, J. Chem. Phys., 44, 4005 (1966).

46. K. E. G. Palilus, Mol. Pnys., 10, 381 (1966).

47. B. N. Ganguly, U. N. Upadhyaya, K. P. Sinka, Phys. Rev. I46, 317 (1966).

48. A. M. Bass and H. P. Broica, ed., "Formation and Trapping of Free Raciicals" (Academic Fress, 1960).

49. See M. Onchin, Adv. in Catalysis, 16, 2 (1966).

50. (a) R. E. Glover and H. T. Coffey, Rev. Mod. Phys., 36, 299 (1964). 
(b) A. P. Smirnov, V. N. Totubolin, and I. S. Parshina, Soviet Phys. JETP, 22, 84 (1966).

51. R. F. Broom and E. H. Rhoderick, Phys. Rev., 116, 344 (1959).

52. R. D. Chaudhuri and J. B. Brown, Phys. Rev., 139A, 1482 (1965).

53. J. N. Bremer and V. I. Newhouse, Phys. Rev., I16, 308 (1959).

54. See especially (a) Ref. 50a, but also (b) V. L. Newhouse, "Applied Superconductivity" (John Wiley and Sons, 1964).

55. R. W. Roberts and T. A. Vanderslice, "ultrahigh Vacuum and Its Applications" (Prentice-Hall, 1963).

56. CRC - Handbook of Chemistry and Physics (43rd Edition).

57. General reference to UHV technology

(a) Ref 55; (b) A. Guthrie, "Vacuum Technology" (John Wiley and Sons, 1963).

58. Most of our results have been reported in

(a) H. M. McConneII, B. M. Hoffman, D. T. Thomas and F. R. Gamble, Proc. Nat. Acad. Science, 54, 371 (1965)

(b) B. M. Hoffman, F. R. Gamble and H. M. McConneII, J. Am. Chem. Soc., to be published.

sy. A. Kwiram, I'hesis (California Lnstitute of l'echnology).

60. Andonian Assoc., Waltham, Mass.

61. Varian Assoc. Bulletin \#Vac 2224, by Burdell E. Keitzman.

62. Ultek Corp. Technical Literature Reprints, \#DII41.

63. C. Kittel, "Introduction to Solid State Physics" (John Wiley and Sons, 1956).

64. H. I. Caswell, J. Appl. Phys., 32, 105 (1961).

65. Private communication obtained through Canven Mead.

66. W. Runl, Z. Physik., 157, 247 (1959).

67. J. C. Suits, Phys. Rev., 131, 588 (1963).

68. Taken from A. Morette, Vanadium, in P. Pascal, "Nouveau Traite de Chimie Minerale,"VoI. XII. 
69. L. Meyers and W. A. Littie, Phys. Rev. Letters, 13, 325 (1964).

70. Temperature Physics, 1962, ed. R. O. Davies (Butterworths, 1963).

71. W. OpItz, Z. Pnys1k., 141, 263 (1955).

72. V. L. Ginzburg, Soviet Phys. Doklady, 3, 102 (1958).

73. (a) J. E. Mercereau and T. K. Hunt, Phys. Rev. Letters, 8, $243(1962)$.

(b) Ref. 52 .

74. (a) Crittenden, Cooper, Schmidlin, and Leam, D. 282, "Structure and Propenties of Thin Fizns," ed. by C. A. . Newhouse et aI. (John Wiley and Sons, 1959).

(b) Ref. 54, b.

75. R. S. Mulliken, J. Chem. Phys., 2, 782 (1934); 3, 573 (1935).

76. For recent reviews of inter-molecular charge-transfer systems see:

(a) R. S. Mulizker and w. B. Person, Ann. Rev. Phys. Chem., 13,107 (1962).

(b) G. Briegleb, "Elektronen-Donator-Acceptor-Komplexe" (Springer-Verlag, 196I).

77. We have preparec charge transfer complexes of TMP with the acceptors tetracyanoethylene and 2,3-dichloro-5,6-dicyanobenzoquinone. A derived value of I for TMP is listed in Table I.

78. R. Z. Glover and M. D. Sherrill, Phys. Rev. Letter, 5,248 (1960).

79. H. I. Stadler, Phys. Rev. Ietters, 14, 979 (1965).

80. W. RHHI, p. 475, IX International Conference on Low Temperature Physics, ed. J. G. Daunt, et al. (Plenum Press, 1965).

81. D. O. Hayward and B. M. W. TrapneII, "Chemisorption" (Butterworths, 1964).

82. E. Bucher, F. Heinigen, anci J. Mulier, P. 1059, IX International Conference on Low Temperature Physics, ed. J. G. Daunt, et al. (Plenum Press, 1965). 


\section{Abstract}

It is proposed that the method of photoselection be usec in studying the ESR of the triplet states of randomiy oriented molecules in rigid glasses.

l'he originat observations of the ESR of organic molecules in tine lowest triplet state were made on single crystals of solid solutions of naphthalene in durene. The spectra are due to transitions between sublevels of the triplet and are highly anisotropic because of the zero-field splitting (I). This anisotrofy makes it very difficlilt to observe the ESR of a randomly oriented sample of such molecules. The half-field, $/ \Delta m /=2$ tnansitions are comparatively isotropic and have been observed with nononiented molecules in rigid glasses (2). Recently, the $/ \Delta \mathrm{m} /=1$ transitions have also been seen in glasses, but the intensities are much lower (3). Howeven, observation of the higher field resonarce allows more ready calculation of the zero-field splitting parameters and is more sensitive to the values of these parameters.

It is therefone proposed that the method of photoselection be used fon high field ESR experiments on triplet states (4). Photoselection relies on selecting with polanized light a non- 
random array of molecules from a randomly oriented sample. If the light is chosen to correspond to an absorption band with known polarization, the excited molecules produced now have a known orientation in space. Although molecules not parallel to the polarization axis can still absorb light with a probability proportional to the square of the cosine of the angle between the transition dipole and the electric vector, there is still an important net orientation. This is most recently shown by . F.I-Sayed and Pavlaponlos in triplet-triplet ahsorption studies using photoselection (5).

The normal high field spectrum from randomly oriented molecules in a rigid glass is three pairs of lines (3). Each pair arises from a group of molecules in which one of the three principle axes of the zero-field splitting tensor is parallel to the magnetic field. At these three canonical orientations the zero-field splitting takes on a stationary value as a function of orientation to the magnetic field. This is why the ESR absorption can be seen despite the large anisotropy. However in non-canonical orientations the splitting varies rapidly with small changes in orientation. The signals from these molecules therefore contribute to the noise level.

If the polarized light is oriented so that the absorbing molecules are in a canonical orientation, the pair of lines that correspond to that orientation should be effectively enhanced, while the lines from the other two orientations should largely 
disappear. If the direction of the electric dipole transition moment is parallel to one canonical direction, such a situation will be the case when the electric field io parallcl to tho magnetic field. Placing the electric field perpendicular to the magnetic field will then allow the observation of the other two pairs of lines, and the pair seen in the parallel case will be suppressed. Then if the orientation of the transition dipole moment with respect to the molecule is known, this makes it . possible to relate a given splitting to the molecular orientation involved, and therefore to relate the axes that diagonalize the zero-field tensor to the molecular axes.

Added Note: This suggestion was originally submitted as a candidacy proposal in February, 1964. Since that time Kottis and Lefebvre [J. Chem. Phys. 4I, 366 (1964)] published a note containing computer simulated spectra indicating that the effect of photoselection would indeed be to enhance the resonance of certair canonical orientations with respect to the othors. Thcy also point out that if the axes of the zero-field tensor are known with respect to the molecular axes, information about the transition-dipole moment can be obtained.

Very recently, Lhoste, Haug, and Ptak used the combination of photoselection and magnetic resonance and experimentally known orientations of the zero-field tensor to experimentally obtain information on the transition moments in phenoxazine [J. Chem. Phys. 44, 648 (1966)] and in coronene and phenanthrene 
[三ic., p. 655]. Shortly thereafter, El-Sayed and Siegal ilbid., p. 1416] used the known directions of polarization of the electronic absorption bands of phenanthrene- $d_{10}$ to assign the canonical directions for the zero-field tensor. In addition, they discuss in detail the method of making such assignments. 


\section{REFERENCES}

1. C. A. Hutchison and B. W. Mangum, J. Chem. Phys., 29, 952 ( $\perp$ YS8); ibid., 34, 908 (1961).

2. J. H. van den Waals and M. S. de Groot, Mol. Phys., 2, 333 (part I) (I959); M. S. de Groot and J. H. van der Waals, Mol. Phys., 3, 190 (part II) (1960); M. S. de Groot and J. H. van der waals, Mol. Phys., 6, 545 (part III) (1963).

3. W. A. Yager, E. Wassenman, and R. M. R. Cramer, I. Chem. Phys., 37, 1148 (1962).

4. A. C. Albrecht, J. Mol. Spec., 6, 84 (I96I).

5. M. A. El-Sayed and T. Pavlapoulos; J. Chem. Phys., 39, 834 (1963). 
PROPOSITION II

\begin{abstract}
We propose that low temperature optical absorption measurements be performed on crystalline 1:1 charge transfer complexes in order to determine experimentally the low temperature state of these crystals and how il develops with increasing temperature.
\end{abstract}

There have been extensive experimental and theoretical studies of the electrical and magnetic properties of crystalline I:I complexes DA between aromatic donon molecules $D$, and acceptor molecules A (1). Recently, McConnell (2) has proposed a treatment of the ground state and elementary excitations in the limit of low temperatures, although, as will be discussed, room temperature properties as determined by optical $(3,4)$ spectroscopy seem to agree well with its predictions. We will propose here an extension of these measurements in order to explain the nature of this agreement, thereby obtaining the experimental results necessary for an extension of the present theory of these complexes.

On the basis of a simple molecular field model, Mcconnell

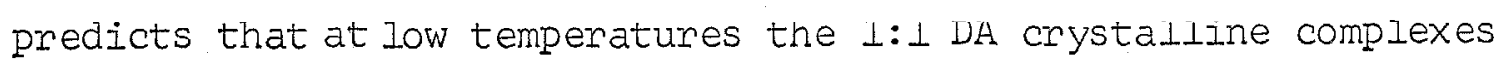
must divide sharply into two classes. Nonionic crystals (N) will be composed of essentially neutral components; ionic crystals (I) 
will be composed of positive and negative ion radicals. Both ciasses should have a nonmagnetic ground state, the former because the neutral components are diamagnetic; the latter because the spin exchange interaction between radicals is expected to be antiferromagnetic. For Doth classes, the lowest energy excitations above the ground state are expected to be magnetic, being triplet excitions with large excitation energy in ciass $\mathrm{N}$, and thenmally accessible linear spin waves for ciass I.

Optical absorption and paramagnetic rcoonance expcrimento have indicated that at room temperature there coes seem to be such a sharp division of these sclids into diamagnetic, nonionic complexes and, at least partially paramagnetic, ionic complexes. Furthermore, the paramagnetism of the I complexes decrease exponentially with decreasing temperature, indicating that it is associated with a thermal excitation of the crystal (5).

These facts seem to be in good agreement with the theory. However, it must be stressed that the tineory as formulated is only for low temperatures, whereas the IR and visible experiments which verify the division into classes are room-temperature measurements. In particular, the following questions may be askea. Are all those crystals which are ionic at room temperature also ionic at absolute zero, of is the room temperature iunil state, of at ledsh sune, ine resuli uf liferlial excilalions? If some compiexes change from $N$ to $I$, is this change continuous or abrupt? Extension of the theory to highen temperatures can 
be made along the same lines as work by Chesnut (6) on triplet exciton systems. His results suggest that at high temperatures cormesponding to high excitation concentrations, a rafid or even discontinuous change of the degree of ionization may cccur. Finaliy, is the temperature dependence of the paramagnetism related lo a curresponding change in ionization?

We propose that these questions can be answered by measuring the visible and/or IR spectra of several complexes down to cryogonic temperatures. Both techniques can differentiate innic from nonionic crystals, and can tell whether, and in what manner, the degree of ionization of a complex changes with decreasing temperature. Furthermore, any changes in ionization can be compared to known, or measurable, changes in paramagnetism.

A preliminary IR experiment has been performed in coilaboration with $\mathrm{Mn}$. E. Weissbergen. We measured the spectrum of the ionic 1:1 complex p-phenylenediamine-chloranil (7) from room temperature to $\sim 16^{\circ} \mathrm{K}$ and found no evidence for a decrease in the degree of ionization. Fowever, tine spectna were taken with the complex dispersed in a KBr pellet, and paramagnetic resonance measurements showed that this technicue produced appreciable decomposition. The cecomposition products are presumably $D^{+}$and $A^{-}$radicals. Therefore, the results of this experiment are by no means conclusive. Howcver, tho usc of Nujol as a dispensing medium should reduce decomposition as a problem. 
REFERENCES

1. Fon a review see R. S. Nulliken and W. B. Person, Ann. Rev. Phys. Chem., 13, 107 (1962).

2. H. M. McConnelI, B. M. Hoffman and R. M. Metzgen, Proc. Nat. Acad. Sci., bs, 4o (1965).

3. R. Foster and T. J. Thompson, Trans. Far. Soc., 59, 296 (1963).

4. Y. Matsunaga, J. Chem. Phys., 4I, 1609 (1964).

5. Scc, for example, A. Ottenberg, C. J. Iloffman, and J. Osiecki, J. Chem. Phys., 38, 1898 (1963).

6. D. B. Chesnut, J. Chem. Phys., 40, 405 (1964).

7. Prepared by Dr. R. C. Hughes by co-subIimation of the components. 


\title{
PROPOSITION III
}

\begin{abstract}
It is proposed that attempts be made to measure a Knight shift for the alkali metali metals in alkali metal-graphite intercalation compounds. Measurement of carbon-I3 shifts are mentioned as a possible alternative. Some suggestions are offered for the study of other graphite intercalation compounds:
\end{abstract}

Hannay et al. (I) have recently discovered that the potassium, rubidium, and cesium intercalation compounds of graphite in which graphite carbon layers alternate with layers of metal atoms are superconductors. However, compounds in which a metal layer alternates with two graphite layers show no signs of superconductivity. These facts were related to theoretical studies (2) which suggest that superconductivity cannot occur in less than three dimensions. The implication is that the addition of a second graphite layer between metai layers may sufficiently restrict the conductivity to two dimensions to prevent superconductivity.

After a brief discussion of the alkali metal intercalation compounds, we suggest some measurements which should give information about their electronic properties, and shed light on the changes in conductivity and superconductivity with composition. 
Graphite can form binary intercalation compounds with the alkali metals and with a large number of molecules. We here consider the spontaneous lamellar compounds as described in the review article by Hennig (3). These compounds can exist in several definite concentration states which differ in the number of graphite layers separating intercalated layers of reactant. Fulassiun yraplite, fon exdnple, edr exlibil ordered structures from stage 5, which has periodically anranged potassium layens separated by five carbon layers, through stage 1 , in which car-. bon layers alternate with potassium layers.

The electrical resistivity of single crystals of graphite is about 4 to $6 \times 10^{-5} \Omega$-cm. parallel to the planes (a-axis), which corresponds to that of a poor metal. Perpendicular to the planes (c-axis), the resistivity is much higher, being $\lesssim .2 \Omega \mathrm{cm}$. (4). The lamellar compounds are more "metallic" than graphite; They exhibit large decreases in resistivity as the reactant concentration is increased (2). The a-axis resistivity decreases steeply at the stage of dilute compound formation, where the intercalated reactant layers are widely separated, and then levels off; the resistance decrease for graphite potassium is about I/10, and this value is reached at an approximate composition $\mathrm{C}_{50} \mathrm{~K}$. The $\mathrm{c}$-axis resistance decreases more slowly, but continues decreasing through the entire concentration range. By stage 1 , corresponding to $C_{8} K$, the total change is a factor of .003 . 
Changes in the in-plane electrical properties can be qualitatively understood in terms of a two-dimensional band model (5). There appears to be a transfer of electrons between graphite and reactant, leaving the reactant partially on totally ionized. Since the two-dimensional density of states is very low at the top of the Fermi level and increases both above and below, reactants which either add or withdraw electrons increase the conductivity. A three-dimensional theory is clearly necessary * to explain the changes in o-axis conductivity.

The fact that there is a full order of magnitude decrease in the c-axis resistivity of potassium graphite upon going from stage 2 to stage $I$, whereas the a-axis resistivity remains rough Iy constant over this concentration range, might be used to support the position that full three-dimensionality is a requisite to superconductivity. The additional metal atoms in stage I may merely add an additional, necessary number of carriers to the graphite layers, but the constancy of the a-axis conductivity from stage 2 to stage 1 must then be explained.

As a means of determining the electronic structure of the alkali metal graphite compounds, we propose the application of nuclear magnetic resonance techniques. In particular, if the bonding between reactant and carbon layers is really "metallic" the reactants should exhibit a Kright shift $(G)$. Mhis pllenomenon is a shift of NMR lines in metals (at fixed frequency) to fields lower than those in the related ion. This shift is usually an order of magnitude, or more, larger than ordinary chemical shifts. 
It arises from the action of the Pauli paramagnetism of the conduction electrons on the nuclei via the electron-nuclear contact hyperfine interaction.

The fractional shift, $\Delta \mathrm{H} / \mathrm{H}_{\mathrm{O}}$, is proportional to the average value at the Fermi level of the s-conduction electron wave functions taken at the nuclear pooition $\left\langle\left|u_{k}(0)\right|^{2}\right\rangle_{a v}$, and the Pauli susceptibility, $\chi_{p}$. For a cubic environment

$$
\frac{\Delta H}{H_{0}}=\frac{8 \pi}{3}\left\langle\left|u_{k}(0)\right|^{2}\right\rangle_{\text {aw }} X_{p}
$$

Since the Knight shift is thus sensitive to the properties of the conduction electrons, its determination through the full alkali metal concentration range should tell how bonding changes with concentrations. In particular, any changes found as the stoichiometry is changed from that of stage 2 to that of stage 1 might bear on the superconductivity results.

Unfortunately, potassium, rubidium and cesium all have ruclear spins of yrealer lian $1 / 2$ and muderalely larye quadrupule moments. Ordinarily the presence of a large quadrupole moment in a non-cubic environment, as exists in these compounds, would broaden the NMR signal into indetectability. However, rapid motion will average the quadrupole interaction to zero. In the intercalation compounds, particularly at high temperatures, it is very possible that the metal atoms will be able to diffuse readily enough that their motion will provide the necessary averaging, particularly at higher dilutions. Support for this. 
is derived from $X$-ray diffraction studies which indicate that the metal atoms in stage 2 compounds are mobile. (7).

Because of the non-cubic envirument, he knight shift will be anisotropic, which will also cause broadening. Anisotropic Knight shifts have been observed ( 8 ), however, and this factor should be insignificant relative to quadrupole effects. .

An alternative (expensive) possibility is to prepare carbon-13 enriched graphite by the pyrolysis of enriched methane, having no quadrupole moment. The conduction electrons on the carbon atoms are primarily in p-type orbitals; this would reduce $\left\langle\left.\mu_{k}(0)\right|^{2}\right\rangle_{a r}$, and therefore the Knight shift. Nevertheless, the effects should still be separable from ordinary chemical shifts.

Although the alkali metal compounds are perhaps the must interesting, it should be mentioned that some of the molecules which form intercalation compounds have nuclei with spin $1 / 2$, and therefore no quadrupole moment to interfere with NMR measurements (1). Other molecules, such as ferric chloride, have nuclei which exhibit Mossbauer resonance, which also exhibits shifts with changes in electron density. 
1. N. B. Hannay, T. H. Gebaile, B. T. Matthias, K. Andres, P. Schridt, and P. Macilair, Phys. Rev. Letters, 14, 225 (1965).

2. R. A. Ferrell, Pnys. Rev. Letters, 13, 1330 (1964).

3. G. R. Hennig, Proo. in Inorg. Chem., I, 125 (1959).

4. I. C. E. Blackman, J. F. Nathews, and A. R. Ubbelchde, Proc. Roy. Soc. A, 258, 339 (1960).

5. (a) P. R. Wallace, Phys. Rev., 71, 622 (1949).

(b) C. A. Coulson anci R. Maylor, sroc. Phys. Soc. A, 65, 815 (I952).

6. (a) W. D. Knight, Solid State thysics, 2, 93 (1956).

(b) C. P. Slichter, "Principles of Viagnetic Resonance," Harper \& Row.

7. N. Rildonff, Adv. in Inong. Chem. and Radiochem., 223, vol. I (1959); see p. 240 .

8. N. Bloembergen and G. Rowlanc, Acta Net., I, 731 (-953). 
PROPOSIIION IV

\begin{abstract}
The linear polyphenyls ane consiciered as a model system for the attractive electron-electron-interaction due to conductive conjugation. The syster. is not found to satisfy the criterion for superconductivity.
\end{abstract}

The linear polyphenyls are an obvious rodel polymer for calculating the attractive interactions between e-iectrons due to "conductive conjugation" ( 1 ). The plane of symetry perpendicular to the rings and parallel to the chain allows a classification into symetric "conduction" orbitals and antisymmetric, "locaized" orbitals; the lattor provide the statos noccssary to produce an attraction between electrons in the former. After solving in the Hückel approximation for the energies and wave functions, we follow little (2) in determining the criterion for superconductivity and in evaluating the matrix elements for a screened coulomb repuision and fon the attractive interaction.

We consider a polyphenyl of $\mathrm{N}$ units with cyclic boundary conditions: ring $N$ is bonded to ring 1. We chose the "natural" unit of one phenyl ring as our unit cell, numbering the atoms as follows: 


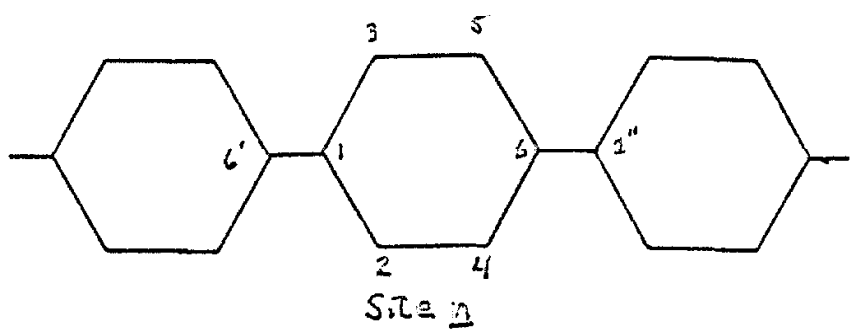

As a corbequerce of lhe cyclic boundary conditions the wave functions and energies can be characterized by the pseudomomertum, $k$.

This, with the refiection symmetry throlugh the center of the chain, allows us to form the symmetry-adapted orbitals

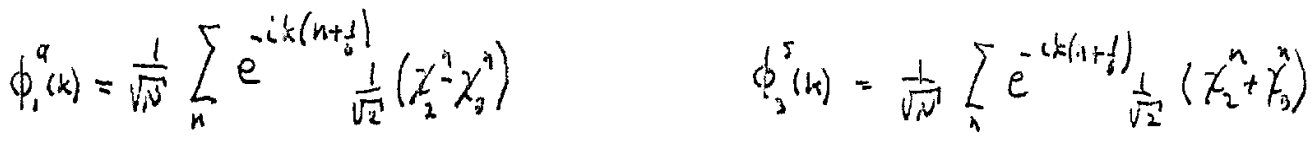

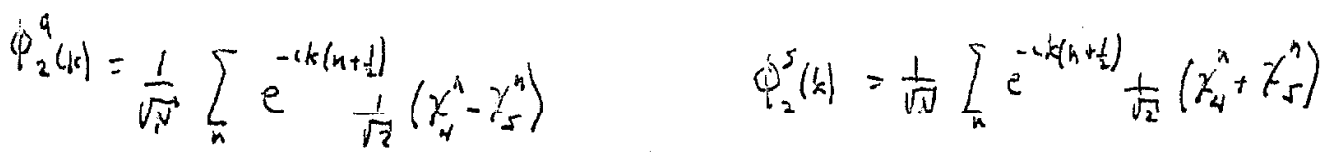

$$
\begin{aligned}
& \phi_{1}^{5}(c)=\frac{1}{\sqrt{3}}\left[e^{-\operatorname{ck} k n} \gamma_{1}^{1}\right. \\
& \phi_{4}^{s}(k)=\frac{1}{\sqrt{0}} \sum_{n} e^{-i t(n+3)} y_{0}^{n}
\end{aligned}
$$

Here $x_{i}^{n}$ is a carbon $2 p-\pi$ orbitaz on ring r., carbon $i$. The aorbitals ane antisymmetrie, the s-orbitals symmetric with respect to reflection. We follow the procedure of measuring enengies, $\varepsilon_{k}$, in units of $\beta$, the resonance integral, with respect to a zerc of energy at $a$, the coulomb integrä. To take into account the possibility of noncoplanarity of the benzene rings, on of longer bonds between them, the interring resonance integral is taken to be $\beta^{\top}$, such that $\beta^{\prime} / \beta=\delta<1$. 
The seclilar determinant for the antisymmetric orbitais is

$$
\left|\begin{array}{cc}
\epsilon_{x} & e^{-k k / 3} \\
e^{k / 3} & \epsilon_{x}
\end{array}\right|=0
$$

which gives

$$
t_{k}= \pm 1 \quad \text { (independent of } k \text { ) }
$$

The antisymmetric crbitals on one ring do not have matrix elements to those on other rings, and therefore, as might have been expected, they have the same energies as would those on an isolatec Denzene ring. These two levels, separated by $\bar{h}_{w}=2 \beta$, form the system which will couple electrons in the symmetric orbitals.

The secular deteminant for the symmetric orbitals is

$$
\left|\begin{array}{cccc}
t_{k} & \delta e^{i k / 3} & \sqrt{2} e^{-i k / 3} & 0 \\
\delta e^{-i k / 3} & t i & 0 & \sqrt{2} e^{-i k / 3} \\
\sqrt{2} e^{i t / 6} & 0 & \epsilon_{k} & e^{-i k / 3} \\
0 & \sqrt{2} e^{-i k / 3} & 2^{-i k / 3} & \epsilon_{x}
\end{array}\right|=0 \quad \text { (4) }
$$

giving

$$
\begin{aligned}
& \epsilon_{k}^{4}=\left(\delta+\delta^{2}\right) \epsilon_{k}^{2}+(4+\delta)^{2}-4 \delta \cos k=0 \\
& \epsilon_{k}^{2}=\frac{5+\delta^{2}}{2} \pm 2\left[\left(\frac{\delta^{2}+3}{4}\right)^{2}+\delta \cos k\right]^{1 / 2} \cdots \quad-\pi \leqslant k \leqslant+\pi \\
& =3 \pm 2[1+\cos k]^{\frac{1}{2}} \quad \text { if } \delta=1
\end{aligned}
$$


l'hus, the two pairs of symmetric orbitals in benzene, one pain at $\varepsilon= \pm 1$, the othen pair at $\varepsilon= \pm 2$, broacen into two pairs of bands as $N \rightarrow \infty$ in the polyphenyl. The lowen two s-bands and the lower a-band are completely filled, the other bando completely empty, in the neuträ molecule. The energy gap separating the filled and unfilled orbitals is

$$
\begin{aligned}
y & =2\left\{\frac{-+\delta}{2}-2\left[\left(\frac{\delta^{2}+3}{4}\right)^{2}+\delta\right]^{1 / 2}\right\}^{1 / 2} \beta \\
& =2(3-2 \sqrt{2})=.834 \beta \quad \text { if } \delta=1
\end{aligned}
$$

If we simplify to $\delta=1$, the lower two s-bands touch at $k= \pm \pi$, as do the upper two. Then, by using the extencied zone scheme, we get two bands, separated by the energy gap $\checkmark$, with limits on $k,-2 T \leqslant k \leqslant+2 \pi$.
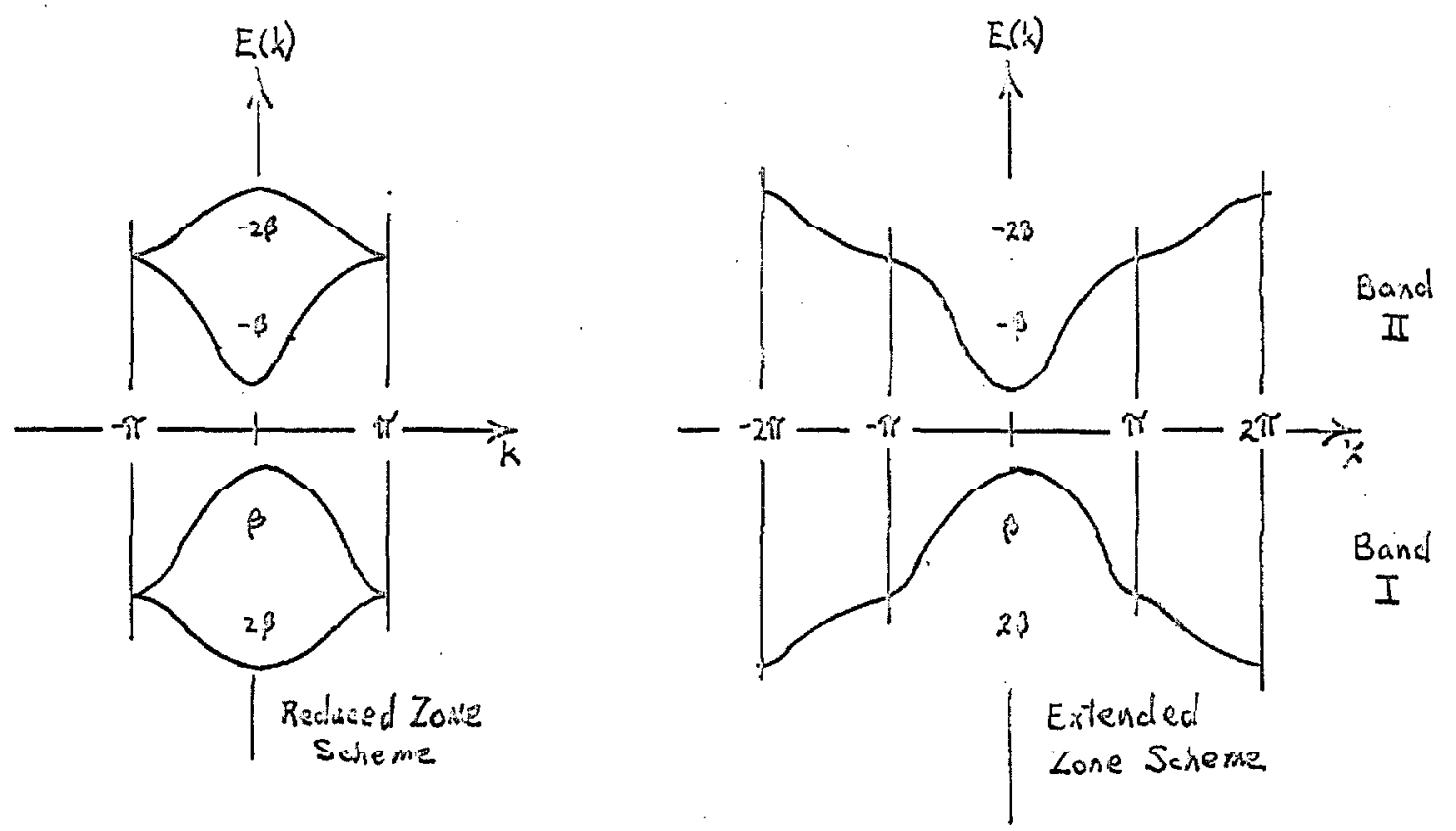
The energy can be rewritten

$$
\frac{x}{I}(t)=-\left[2 \sqrt{2} \sin ^{2} \frac{k}{4}+\left(\frac{y}{2}\right)^{2}\right]^{1 / 2}
$$

Using this expression Eon the energy, we can eithen integrate Little's equation 3.1 without approximation, on use his equation 3.4 to give the criterion for superconductivity in the presence of the gap, $\gamma$. The integration, with the upper limit $\bar{\hbar}$ gives

$$
\begin{aligned}
|V|=\left|V_{\text {couc }}+V_{\text {attr. }}\right| & \geqslant 1.298 / \mathrm{N} \\
& \approx 2.74 \div \mathrm{V} / \mathrm{N}
\end{aligned}
$$

where we take $\beta=-2.2 \mathrm{ev}$. Of course the sign of $\mathrm{V}$ musl be negative.

We must now calculate the coulomb repulsion and electroncoupled attraction tcrmo for the o-etatco. The o-statce of importance are those where the semiconducting gap is smallest, near $k=0$, so that promoting an electron from the Filied to the infilled band costs the least energy. Therefore, matrix elements need only be cazculated for s-states with $k \rightarrow 0$. Tren, since the intenactions between $s$ and a-states conserve momentum, onIy those a-states with $k \rightarrow 0$ occur in matrix elenents. For $k=C$, the wavefunctions are just sums of the related benzene wave function for the individuã sites. The s-band functions are 


$$
\begin{aligned}
& \psi_{I}(0)=\frac{1}{\sqrt{N}} \sum_{n} \frac{1}{\sqrt{2}}\left[\varphi_{3}^{3}(0)-\phi_{4}^{S}(0)\right]+\frac{1}{\sqrt{3}}\left[\theta_{1}^{S}(0)-\phi_{2}^{s}(0)\right] \\
& \psi_{I}(0)=\frac{1}{\sqrt{2}} \sum_{n} \frac{1}{\sqrt{17}}\left[\phi_{7}^{5}(0)+\phi_{4}^{5}(0)\right]-\frac{1}{\sqrt{3}}\left[\phi_{1}^{5}(0)+\phi_{2}^{5}(0)\right]
\end{aligned}
$$

The a-Dand functions ane

$$
\begin{array}{ll}
\left.\psi_{B} \sum^{\prime} \theta\right)=\frac{1}{\sqrt{2 N}} \sum_{A}\left[\phi_{A}^{\prime}(0)-\phi_{A}^{2}(0)\right] & t=-\beta \\
\psi_{A 1}(0)=\frac{1}{\sqrt{2 N}} \sum_{n}\left[\phi_{A}^{\prime}(0)+\phi_{A}^{2}(0)\right] & t=+\beta
\end{array}
$$

In evaluating the coulomb repulsions jetween s-state electrons we follow Iittle; we assume that only one-center tems contribute and that these are screened. In the one-center approximation all contributing coulomb matnix elements are equal:

$$
\begin{aligned}
& V_{\text {cout. }}=(I I \mid I I)_{\text {scr. }}=(I I \mid I I J)_{s c r}-(I I \mid I I I)_{s c r} \\
& =\int \psi_{(1)}^{J} \psi_{(1)}^{\mp} \frac{e^{-\lambda r_{12}}}{r_{12}} \psi_{(2)}^{\mp} \psi_{(2)}^{I} d_{r}^{3} r_{1}^{\prime} r_{2}=\frac{1}{4 N} \int\left|r_{(1)}\right|^{2} e^{-\lambda r_{12}}\left|\gamma_{(2)}\right|^{2} d r_{12} \\
& =1.5 \mathrm{e} / \mathrm{N}
\end{aligned}
$$

We have used Little's approximation in evaluating tine one-center integral.

The matrix element for the attnactive interaction involves the scattering of a "symmetric" electron from bard I to band II with the excitation of an "antisymmetric electron from $A$ i to $A 2$, anc the transfer of wavevector $Q$. We aydin consider the limit $k, Q=0$. We consider this interaction to be unscreened. We 
take only one-center terms, and evaluate them in the same way that Little evaluated the screened integrals. Then,

$$
\begin{aligned}
\frac{U_{\text {atr. }}}{N} & =\int \psi_{A_{2}}(1) \psi_{d}(1) \frac{1}{r_{12}} \psi_{I^{2}}(2) \psi_{I}(2) d^{3} r_{1} d_{r_{2}}^{3} \\
& =\frac{083}{N} \int\left|\psi_{i}(1)\right|^{2} \frac{1}{r_{12}}\left|\psi_{i}(2)\right|^{2} d^{3} r_{12} \\
& =1.41 \mathrm{eV} / \mathrm{N}
\end{aligned}
$$

We recall tine formia for the attractive interaction

$$
V_{a+i r .}=\frac{1}{N} \frac{2 T \omega\left|U_{a+r i r}\right|^{2}}{(\Delta \epsilon)^{2}-(h \omega)^{2}}
$$

Here $\hbar w=2 \beta ;$ because of the semiconducting enengy gap, $\Delta \varepsilon=$ $=.8348$. Thus the gap actually enhances the effectiveness of the interaction. Agair using $\beta=-2.2 \mathrm{eV}$, and using the result in equation 11, we get for equation 12

$$
V_{\text {utr }}=-\frac{\left|U_{0 . t_{r}}\right|^{2}}{1.8 \mathrm{~N}} \cdot \mathrm{V}=-1.2 \mathrm{eV} / \mathrm{N}
$$

Thus,

$$
\begin{aligned}
\langle v\rangle & =V_{\text {coub }}+V_{\text {atir }} \\
& \left.=\frac{1.5}{. v}-\frac{1.2}{N}=+03 \mathrm{eV} / \mathrm{N}\right\rangle 0
\end{aligned}
$$

Therefore, our mode: does not merely fail to satisfy the necessary inequality, equation 7 ; the interaction is not even attractive. In fact, it can be seen that not even complete neglect of coulorb repulsions would be sufficient to satisfy equation 7 . 


\section{REFERENCES}

1. H. M. McConnelI, unpublished.

2.W. A. Little, Phys. Rev., 134A, I416 (1964). All references to Little's work ane to this paper. 


\title{
PROPOSITION V
}

\begin{abstract}
We propose to study the interaction of paramagnetic copper (II) ions with DNA by measuring the water proton reiaxation enhancement and changes in phosphorous-31 nuclear magnetic resonance upon binding of the copper.
\end{abstract}

Eichhorn and Clark (I) have recently studied the effect of copper(II) ions on the thermal denaturation. of DNA. They found that the presence of cupric ion causes the unwinding of the DNA helical structure, and that the unwound strands can apparently be totally mewound into the native double-helical configuration. We propose that nuclear magnetic resonance (NMR) measurements of the effects of Dincing copper ions to DNA on the relaxation rates of water protons (2) and of DNA phosphorous atoms (3) can give impontant information about the nature of the binding. We finst discuss briefly the rature of the measurements. A paramagnetic ion in water increases the longitudinal proton relaxation time for those molecules in its hydration sphene. (The inyoiration sphere can exchange with the bulk water.) Ipon complexing of the ion, its inuediate environment is changed, and the relaxation rate of the water will also change. A useful expenimental parameter is the relaxation enhancement factor.

$$
\varepsilon=\frac{R^{*}}{R}
$$


$R$ is the relaxation rate due to the ion in water and $R^{*}$ the rate due to the ion in the presence of the binding agent. The observed value of $\epsilon$ will be a weighted average of the enhancement factors. of the free and bound ions. Generally, $\varepsilon>I$ for binding at exterior sites (eg. phosphate groups of DNA) and $c<I$ for binding to sites inaccessible to the water molecules (interion sites). When a paramagnetic ion binds at a phosphate group, the relaxation rate of the phosphomnus-37 nucleus is also changed. If this change is measured in conjunction with the proton enhancement factor it is possible to tell at what ion concentration binding to the phosphates becomes important.

We now discuss some of the characteristics of the DNAcopper (II) ion system, as studied by Eichhorn and ('Lark ( $L(')$. The addition of less than .4 moles of ion per mole of nucleotide increases $T_{m}$, the midpoint of the thermal denaturation curve of DNA. EC suggest this slabilizing effech al low concentrations results from binding to the phosphates. $T_{m}$ is then drastically lowered by increasing concentrations of copper. There is a break in the slope of the curve of $T_{m}$ versus coppen concentration at a molar ratio of 1.5 copper/nucleotide. EC suggest that this may correspond to one copper bound to each phosphate, and an additional copper bound to two nucleotides. However, they reach no definite conclusions about the stoichiometry because they do not know if there exists any unbound copper.

The thermal denaturation (unwinding of DNA strands) of DNA in the presence of copper ions, in solutions of low electrolyte 
concentration, cannot be reversed by cooling. However, upon increase of the ionic strength, the DNA can be totally renatured. The finding that the denaturation by copper (II) can be totally reversed is contrary to usual experience; EC suggest that, while the DNA is cenatured, the single strands are held "in register" by the copper ions.

Finally, the copper denatured DNA seems to be very extenstvely aggregated; hils is suggesled by ils very ligih seäimentation velocity. Their picture of the copper-denatured state contains copper ions coordinated to the bases and interposed between complementary strands, breaking hydrogen bonds and destroying the seconciary structure.

We suggest that proton relaxxation enhancement measurements cominined with phosphorous-3I NMR can supply important information about the nature of the binding of copper (II) ions to DNA. By studying the proton relaxation times for different copper/nucleotide natios it should be possible to determine the number of different kinds of binding sites. Presumably there are at least two, one at the phosphates and one at the Dase itself. If we assume for simplicity that there are only two, then it should be possible to estinate their binding parameters. In any case, phosphorous ivMR should show whether the phosphate site is the first to bind, checking on the suggestion EC made about the natume of the complex at low copper concentration. It is also possible by use of the relaxation measuremento to estimate the fraction of bound copper ions. This information 
permits a check of the stoichiometry of binding at the copper/ nucleotice ratio of 1.5 , where the break in the $T_{m}$ versus copper concentration cunve occurs.

Finally, and perhaps of primary interest, the proton relaxation measurements right give information about the nature of the copper complex in the inghly aggregated denatureci state. If a large fraction of the copper ions bird the bases strands together and keep the bases in register, this fraction may bo oufficicntiy isolated from the water that its enhancement factor would be less than unity, incicative of an interion site. Thus, if one of the binding sites is experimentally found to have $\varepsilon<1$, this will be strong evidence for such binding. 


\section{REFERENCES}

1. G. L. Eichhorn and P. Clark, Proc. Nat. Acad. Sciences, 53, 586 ( 1965$)$.

2. Proton relaxation enhancement measurements are discussed in

(a) J. Eisinger, F. Faway-Estrup, and R. G. Schulnan, J. Chem. Phys., cr2, 43 (2965).

(b) A. S. Mildvan and M. Cohn, Biochemistry, 2, 910 (1963).

3. R. G. Shulman, H. Sternlicht, and B. J. Wyluda, J. Cham. Phye. $43,3116(1965)$. 